\title{
Atmospheric Circulation of Terrestrial Exoplanets
}

\author{
Adam P. Showman \\ University of Arizona \\ Robin D. Wordsworth \\ University of Chicago \\ Timothy M. Merlis \\ Princeton University \\ Yohai Kaspi \\ Weizmann Institute of Science
}

\begin{abstract}
The investigation of planets around other stars began with the study of gas giants, but is now extending to the discovery and characterization of super-Earths and terrestrial planets. Motivated by this observational tide, we survey the basic dynamical principles governing the atmospheric circulation of terrestrial exoplanets, and discuss the interaction of their circulation with the hydrological cycle and global-scale climate feedbacks. Terrestrial exoplanets occupy a wide range of physical and dynamical conditions, only a small fraction of which have yet been explored in detail. Our approach is to lay out the fundamental dynamical principles governing the atmospheric circulation on terrestrial planets-broadly defined-and show how they can provide a foundation for understanding the atmospheric behavior of these worlds. We first survey basic atmospheric dynamics, including the role of geostrophy, baroclinic instabilities, and jets in the strongly rotating regime (the "extratropics") and the role of the Hadley circulation, wave adjustment of the thermal structure, and the tendency toward equatorial superrotation in the slowly rotating regime (the "tropics"). We then survey key elements of the hydrological cycle, including the factors that control precipitation, humidity, and cloudiness. Next, we summarize key mechanisms by which the circulation affects the global-mean climate, and hence planetary habitability. In particular, we discuss the runaway greenhouse, transitions to snowball states, atmospheric collapse, and the links between atmospheric circulation and $\mathrm{CO}_{2}$ weathering rates. We finish by summarizing the key questions and challenges for this emerging field in the future.
\end{abstract}

\section{INTRODUCTION}

The study of planets around other stars is an exploding field. To date, numerous exoplanets have been discovered, spanning a wide range of masses, incident stellar fluxes, orbital periods, and orbital eccentricities. A variety of observing methods have allowed observational characterization of the atmospheres of these exoplanets, opening a new field in comparative climatology (for introductions, see Deming and Seager 2009, Seager and Deming 2010). Because of their relative ease of observability, this effort to date has emphasized transiting giant planets with orbital semi-major axes of $\sim 0.1$ AU or less. The combination of radial velocity and transit data together allow estimates of the planetary mass, radius, density, and surface gravity. Wavelengthdependent observations of the transit and secondary eclipse, when the planet passes in front of and behind its star, respectively, as well as the full-orbit light curves, allow the atmospheric composition, vertical temperature structure, and global temperature maps to be derived — at least over certain ranges of pressure. These observations provide evidence for a vigorous atmospheric circulation on these worlds (e.g. Knutson et al. 2007).

Despite a major emphasis to date on extrasolar giant planets (EGPs), super Earths and terrestrial planets are increasingly becoming accessible to discovery and characterization. Over 50 super Earths have been confirmed from groundbased and spacebased planet searches, including planets that are Earth sized (Fressin et al. 2012) and planets that orbit within the classical habitable zones (HZ) of their stars (Borucki et al. 2012, 2013). Hundreds of additional super-Earth candidates have been found by the NASA Kepler mission (Borucki et al. 2011). For observationally favorable systems, such as super Earths orbiting M dwarfs (Charbonneau et al. 2009), atmospheric characterization has already begun (e.g. Bean et al. 2010; Désert et al. 2011; Berta et al. 2012), placing constraints on the atmospheric composition of these objects. Methods that are used today for EGPs to obtain dayside infrared spectra, map the day-night temperature pattern, and constrain cloudiness and 
albedo will be extended to the characterization of smaller planets in coming years. This observational vanguard will continue over the next decade with attempts to determine the composition, structure, climate, and habitability of these worlds. Prominent next-generation observational platforms include NASA's James Webb Space Telescope (JWST) and Transiting Exoplanet Survey Satellite (TESS), as well as a wide range of upcoming groundbased instruments.

This observational tide provides motivation for understanding the circulation regimes of extrasolar terrestrial planets. Fundamental motivations are threefold. First, we wish to understand current and future spectra, lightcurves, and other observations, and understand the role of dynamics in affecting these observables. Second, the circulationand climate generally_play a key role in shaping the habitability of terrestrial exoplanets, a subject of crucial importance in understanding our place in the Cosmos. Third, the wide range of conditions experienced by exoplanets allows an opportunity to extend our theoretical understanding of atmospheric circulation and climate to a much wider range of conditions than encountered in the solar system. Many of the dynamical mechanisms controlling the atmospheric circulation of terrestrial exoplanets will bear similarity to those operating on Earth, Mars, Venus, and Titan-but with different details and spanning a much wider continuum of behaviors. Understanding this richness is one of the main benefits to be gained from the study of terrestrial exoplanets.

Here we survey the basic dynamical principles governing the atmospheric circulation of terrestrial exoplanets, discuss the interaction of their circulation with global-scale climate feedbacks, and review the specific circulation models of terrestrial exoplanets that have been published to date. Much of our goal is to provide a resource summarizing basic dynamical processes, as distilled from not only the exoplanet literature but also the solar system and terrestrial literature, that will prove useful for understanding these fascinating atmospheres. Our intended audience includes graduate students and researchers in the fields of astronomy, planetary science, and climate-without backgrounds in atmospheric dynamics - who wish to learn about, and perhaps enter, the field. This review builds on the previous survey of the atmospheric circulation on exoplanets by Showman et al. (2010) and complements other review articles in the literature describing the dynamics of solar-system atmospheres (e.g., Gierasch et al. 1997; Leovy 2001, Read and Lewis 2004, Ingersoll et al. 2004, Vasavada and Showman 2005; Schneider 2006, Flasar et al. 2009, Del Genio et al. 2009, Read 2011, as well as the other chapters in this volume).

Section 2 provides an overview of dynamical fundamentals of terrestrial planet atmospheres, including both the rapidly and slowly rotating regimes, with a particular emphasis on aspects relevant to terrestrial exoplanets. Section 3 reviews basic aspects of the hydrological cycle relevant to understanding exoplanets with moist atmospheres. Section 4 addresses the interaction between atmospheric dynamics and global-scale climate, and Section 5 summarizes outstanding questions.

\section{OVERVIEW OF DYNAMICAL FUNDAMEN- TALS}

Key to understanding atmospheric circulation is understanding the extent to which rotation dominates the dynamics. This can be quantified by the Rossby number, $R o=$ $U / f L$, defined as the ratio between advection forces and Coriolis forces in the horizontal momentum equation. Here, $U$ is a characteristic horizontal wind speed, $f=2 \Omega \sin \phi$ is the Coriolis parameter, $L$ is a characteristic horizontal scale of the flow $\left(\sim 10^{3} \mathrm{~km}\right.$ or more for the global-scale flows considered here), $\Omega$ is the planetary rotation rate $(2 \pi$ over the rotation period), and $\phi$ is the latitude.

In nearly inviscid atmospheres, the dynamical regime differs greatly depending on whether the Rossby number is small or large. When $R o \ll 1$, the dynamics are rotationally dominated. Coriolis forces approximately balance pressure-gradient forces in the horizontal momentum equation. This so-called geostrophic balance supports the emergence of large horizontal temperature gradients; as a result, the atmosphere is generally unstable to a type of instability known as baroclinic instability. These instabilities generate eddies that dominate much of the dynamics, controlling the equator-to-pole heat fluxes, temperature contrasts, meridional mixing rates, vertical stratification, and the formation of zonal jets $1^{1}$ On the other hand, when $R o \gtrsim 1$, rotation plays a modest role; the dynamics are inherently ageostrophic, horizontal temperature contrasts tend to be small, and baroclinic instability is less important or negligible. The temperature structure is regulated by a large-scale overturning circulation that transports air latitudinally - that is, the Hadley circulation — as well as by adjustment of the thermal structure due to atmospheric waves. These two regimes differ sufficiently that they are best treated separately.

It is useful to define terms for these regimes. In Earth's atmosphere, the regime of $R o \gtrsim 1$ approximately coincides with the tropics, occurring equatorward of $\sim 20-30^{\circ}$ latitude, whereas the regime of $R o \ll 1$ approximately coincides with the extratropics, occuring poleward of $\sim 30^{\circ}$ latitude. Broadening our scope to other planets, we define the "tropics" and "extratropics" as the dynamical regimesregardless of temperature-where large-scale circulations exhibit $R o \gtrsim 1$ and $R o \ll 1$, respectively.

Figure 1 illustrates how the extent of the tropics and extratropics depend on rotation rate for a typical terrestrial

\footnotetext{
${ }^{1}$ The terms zonal and meridional refer to the east-west and north-south directions, respectively; thus, the zonal wind is eastward wind, meridional wind is northward wind, and meridional mixing rates refer to mixing rates in the north-south direction. A zonal average is an average in longitude.

${ }^{2}$ Except for regions close to the surface, atmospheres are generally stably stratified, meaning they are stable to dry convection: air parcels that are displaced upward or downward will return to their original location rather than convecting.
} 

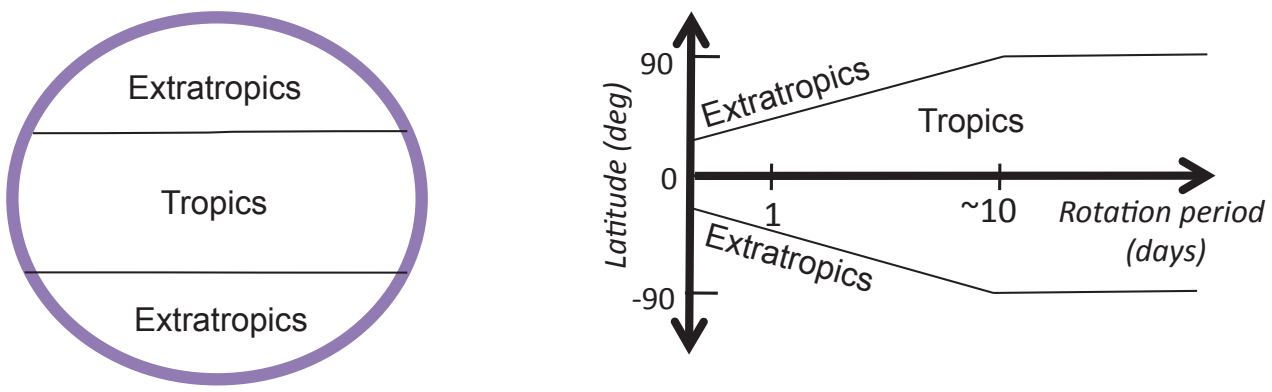

Fig. 1.- Schematic illustration of the regimes of extratropics (defined as $R o \ll 1$ ) and tropics (defined as $R o \gtrsim 1$ ). For an Earth- or Mars-like planet, the boundary between the regimes occurs at $\sim 20-30^{\circ}$ latitude (left panel); however, the transition occurs at higher latitudes when the rotation period is longer, and terrestrial planets with rotation periods longer than $\sim 10$ Earth days may represent "all tropics" worlds (right panel).

planet. The sphere on the left depicts an Earth- or Marslike world where the boundary between the regimes occurs at $\sim 20-30^{\circ}$ latitude. At longer rotation periods, the tropics occupy a greater fraction of the planet, and idealized general circulation model (GCM) experiment $3^{3}$ show that, for Earth-like planetary radii, gravities, and incident stellar fluxes, planets exhibit $R o \gtrsim 1$ everywhere when the rotation period exceeds $\sim 10$ Earth days (e.g., Del Genio and Suozzo 1987) (Figure 1, right panel). Dynamically, such slowly-rotating planets are essentially "all tropics" worlds ${ }^{4}$ Venus and Titan are examples in our own solar system, exhibiting near-global Hadley cells, minimal equator-pole temperature differences, little role for baroclinic instabilities, and a zonal jet structure that differs significantly from those on Earth and Mars. Terrestrial exoplanets characterizable by transit techniques will preferentially be close to their stars and tidally locked, implying slow rotation rates; many of these exoplanets should likewise be "all tropics" worlds.

Figure 2 previews several of the key dynamical processes occurring at large scales on a generic terrestrial exoplanet, which we survey in more detail in the subsections that follow. In the extratropics, the baroclinic eddies that dominate the meridional heat transport (Section 2.1.2 generate meridionally propagating Rossby waves (Section 2.1.3, which leads to a convergence of momentum into the instability latitude, generating an eddy-driven jet stream (Section 2.1.4). Multiple zones of baroclinic instability, and multiple eddy-driven jets, can emerge in each hemisphere if the planet is sufficiently large or the planetary rotation is sufficiently fast. In the tropics, the Hadley circulation (Sec-

\footnotetext{
${ }^{3}$ A GCM solves the global three-dimensional (3D) fluid-dynamics equations relevant to a rotating atmosphere, coupled to calculations of the atmospheric radiative-transfer everywhere over the full 3D grid (necessary for determining the radiative heating/cooling rate, which affects the dynamics), and parameterizations of various physical processes including frictional drag against the surface, sub-grid-scale turbulence, and (if relevant) clouds. "Idealized" GCMs refer to GCMs where these components are simplified, e.g., adopting a gray radiative-transfer scheme rather than solving the full non-gray radiative transfer.

${ }^{4}$ The term was first coined by Mitchell et al. (2006) in reference to Titan.
}

tion 2.2.1 dominates the meridional heat transport; in idealized form, it transports air upward near the equator and poleward in the upper troposphere, with a return flow to the equator along the surface. Due to the relative weakness of rotational effects in the tropics, atmospheric waves can propagate unimpeded in longitude, and adjustment of the thermal structure by these waves tends to keep horizontal temperature gradients weak in the tropics (Section 2.2.2). Many exoplanets will rotate synchronously and therefore exhibit permanent day- and nightsides; the resulting, spatially locked day-night heating patterns will generate largescale, standing equatorial Rossby and Kelvin waves, which in many cases will lead to equatorial superrotation, that is, an eastward flowing jet at the equator (Section 2.2.3. Significant communication between the tropics and extratropics can occur, among other mechanisms, via meridionally propagating Rossby waves that propagate from one region to the other.

We review the extratropical and tropical regimes, along with the key processes shown in Figure 2 in this section.

\subsection{Extratropical regime}

\subsubsection{Force balances and geostrophy}

The extratropical regime corresponds to $R o \ll 1$. For typical terrestrial-planet wind speeds of $\sim 10 \mathrm{~m} \mathrm{~s}^{-1}$ and Earth-like planet sizes, planets will have extratropical zones for rotation periods of a few (Earth) days or shorter. When $R o \ll 1$ and friction is weak, the Coriolis force and pressure-gradient force will approximately balance in the horizontal momentum equation; the resulting balance, called geostrophic balance, is given by (e.g., Vallis 2006. pp. 85-88)

$$
f u=-\left(\frac{\partial \Phi}{\partial y}\right)_{p} \quad f v=\left(\frac{\partial \Phi}{\partial x}\right)_{p}
$$

where $\Phi$ is the gravitational potential, $x$ and $y$ are eastward and northward distance, $u$ and $v$ are zonal and meridional wind speed, $f$ is the Coriolis parameter, and the derivatives are evaluated at constant pressure. The implication is that winds tend to flow along, rather than across, isobars. In 


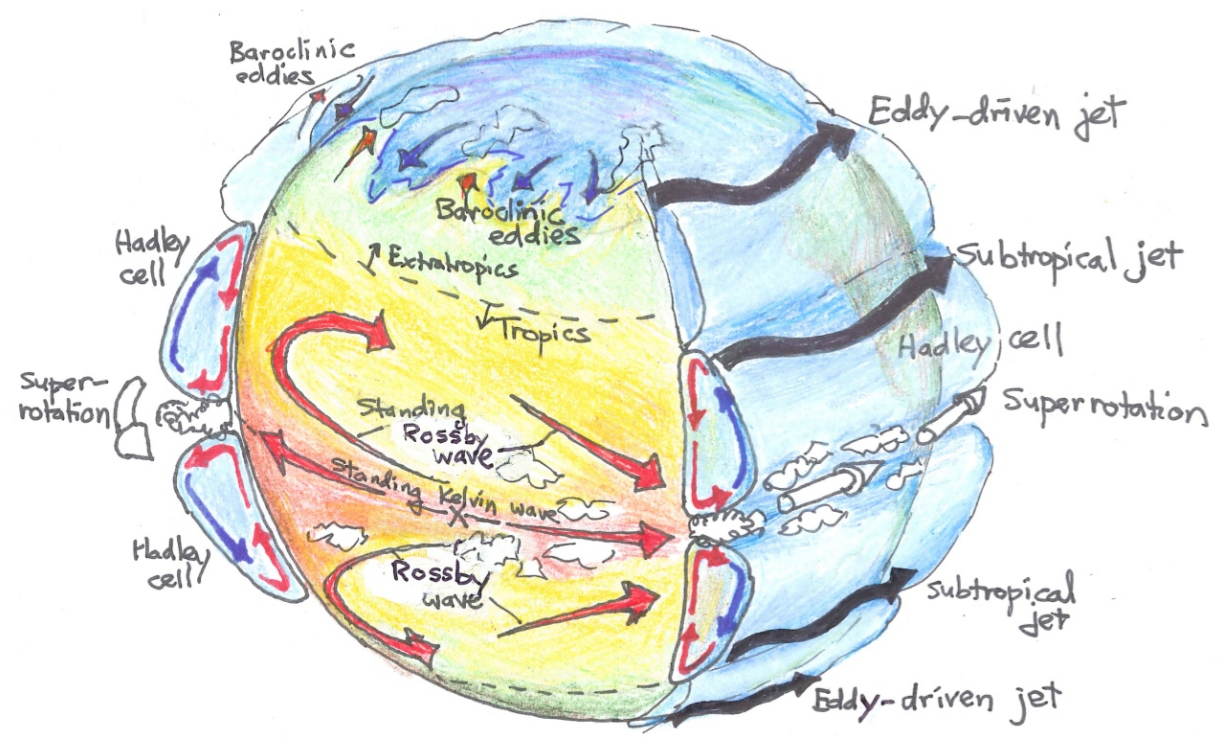

Fig. 2.- Schematic illustration of dynamical processes occurring on a generic terrestrial exoplanet. These include baroclinic eddies, Rossby waves, and eddy-driven jet streams in the extratropics, and Hadley circulations, large-scale Kelvin and Rossby waves, and (in some cases) equatorial superrotation in the tropics. The "X" at the equator marks the substellar point, which will be fixed in longitude on synchronously rotating planets. Cloud formation, while complex, will likely be preferred in regions of mean ascent, including the rising branch of the Hadley circulation, within baroclinic eddies, and — on synchronously rotating planets—in regions of ascent on the dayside.

our solar system, the atmospheres of Earth, Mars, and all four giant planets exhibit geostrophic balance away from the equator, and the same will be true for a wide range of terrestrial exoplanets. See Pedlosky (1987), Holton (2004), or Vallis (2006) for introductions to the dynamics of rapidly rotating atmospheres in the geostrophic regime.

In a geostrophic flow, there exists a tight link between winds and temperatures. Differentiating the geostrophic equations in pressure (which here acts as a vertical coordinate) and invoking hydrostatic balance and the ideal-gas law, we obtain the thermal-wind equations (Vallis 2006. pp. 89-90)

$$
f \frac{\partial u}{\partial \ln p}=\frac{\partial(R T)}{\partial y} \quad f \frac{\partial v}{\partial \ln p}=-\frac{\partial(R T)}{\partial x}
$$

where $T$ is temperature and $R$ is the specific gas constant. The equation implies that, for the geostrophic component of the flow, meridional temperature gradients accompany vertical shear (that is, vertical variation) of the zonal wind, and zonal temperature gradients accompany vertical shear (i.e., vertical variation) of the meridional wind. Because the surface wind is generally weak compared to that at the tropopause, one can thus obtain an estimate of the wind speed in the upper troposphere-given the equatorpole temperature gradient-by integrating (2) vertically.

To order of magnitude, for example, Equation (2) implies a zonal wind $\Delta u \sim R \Delta T_{\text {eq-pole }} \Delta \ln p /(f a)$, where $\Delta T_{\text {eq-pole }}$ is the temperature difference between the equator and pole, $\Delta \ln p$ is the number of scale heights over which this temperature difference extends, and $a$ is the planetary radius. Inserting Earth parameters $\left(\Delta T_{\text {eq-pole }} \approx\right.$
$20 \mathrm{~K}, a \approx 6000 \mathrm{~km}, R=287 \mathrm{~J} \mathrm{~kg}^{-1} \mathrm{~K}^{-1}, f \approx 10^{-4} \mathrm{~s}^{-1}$, and $\Delta \ln p=1$ ), we obtain $\Delta u \approx 10 \mathrm{~m} \mathrm{~s}^{-1}$, which is indeed a characteristic value of the zonal wind in Earth's upper troposphere.

What are the implications of thermal-wind balance for a planet's global circulation pattern? Given the thermal structure expected on typical, low-obliquity, rapidly rotating exoplanets, with a warm equator and cool poles, Equation (2) makes several useful statements:

- It implies that the zonal wind increases (i.e., becomes more eastward) with altitude. Assuming the surface winds are weak, this explains the predominantly eastward nature of the tropospheric winds on Earth and Mars, especially in midlatitudes, and suggests an analogous pattern on rapidly rotating exoplanets.

- Because temperature gradients-at least on Earth and Mars-peak in midlatitudes, thermal-wind balance helps explain why the zonal wind shear-hence the upper-tropospheric zonal winds themselves-are greater at midlatitudes than at the equator or poles. This is the latitude of the jet streams, and thermalwind balance therefore describes how the winds increase with height in the jet streams. Because the tropospheric meridional temperature gradient is greater in the winter hemisphere than the summer hemisphere, thermal-wind balance also implies that the upper-tropospheric, mid-latitude winds should be faster in the winter hemisphere, as in indeed occurs on Earth (Peixoto and Oort 1992) and Mars (Smith 2008). 
- If the mean zonal temperature gradients are small compared to mean meridional temperature gradients, which tends to be true when the rotation is fast (and in particular when the solar day is shorter than the atmospheric radiative time constant), thermal wind implies that the time-mean zonal winds are stronger than the time-mean meridional winds. This provides a partial explanation for the zonal (east-west) banding of the wind structure on Earth and Mars and suggests that a similarly banded wind pattern will occur on rapidly rotating exoplanets 5

The fact that Coriolis forces can balance pressure gradients in a geostrophic flow implies that such flows can sustain larger horizontal pressure and temperature contrasts than might otherwise exist. To order of magnitude, hydrostatic balance of the dynamical pressure and density fluctuations $\delta p$ and $\delta \rho$ (see Holton 2004, pp. 41-42) implies that these fluctuations satisfy

$$
\delta p \approx \delta \rho g D
$$

where $D$ is the vertical scale of the circulation. In the horizontal momentum equation, the pressure-gradient force is approximately $\delta \rho g D /(\rho L)$, where $L$ is a characteristic horizontal length scale of the flow. Noting that $\delta \rho / \rho \sim \delta \theta_{h} / \theta$, where $\delta \theta_{h}$ is the characteristic horizontal potential temperature difference and $\theta$ is the characteristic potential temperature ${ }^{6}$ geostrophy implies (cf. Charney 1963)

$$
\frac{\delta \theta_{h}}{\theta} \sim \frac{f U L}{g D} \sim \frac{F r}{R o}, \quad R o \lesssim 1
$$

where $F r \equiv U^{2} / g D$ is a dimensionless quantity known as a Froude number. For a typical, rapidly rotating terrestrial planet where $U \approx 10 \mathrm{~m} \mathrm{~s}^{-1}, D \approx 10 \mathrm{~km}, g \approx 10 \mathrm{~m} \mathrm{~s}^{-2}$, $f \approx 10^{-4} \mathrm{~s}^{-1}$ (implying rotation periods of an Earth day), the fractional temperature contrasts over distances comparable to the planetary radius approach $\sim 0.1$, implying temperature contrasts of $\sim 20 \mathrm{~K}$. This is similar to the actual equator-to-pole temperature contrast in Earth's troposphere. In contrast, these values greatly exceed typical horizontal temperature contrasts in the tropical regime of $R o \sim 1$ (see Eq. 16.

An important horizontal length scale in atmospheric dynamics is the Rossby deformation radius, defined in the extratropics as

$$
L_{D}=\frac{N D}{f}
$$

\footnotetext{
${ }^{5}$ In the presence of topography or land-ocean contrasts, some local regions may exhibit meridional winds that, even in a time average, are not small compared to zonal winds. In such a case, $u \gg v$ only in the zonal mean.

${ }^{6}$ Potential temperature is defined as the temperature an air parcel would have if transported adiabatically to a reference pressure $p_{0}$ (often taken to be 1 bar). When the ratio of gas constant to specific heat $R / c_{p}$ is constant, as is often approximately true in atmospheres, then it is defined by $\theta=$ $T\left(p_{0} / p\right)^{R / c_{p}}$. It is conserved following adiabatic reversible processes and is thus a measure of atmospheric entropy.
}

where $N$ is the Brunt-Vaisala frequency, which is a measure of vertical stratification. Because the deformation radius is a natural length scale that emerges from the interaction of gravity (buoyancy) and rotation in stably stratified atmospheres, many phenomena, including geostrophic adjustment, baroclinic instabilities, and the interaction of convection with the environment, produce atmospheric structures with horizontal sizes comparable to the deformation radius. As a result, the circulations in atmospheres (and oceans) often exhibit predominant length scales not too different from the deformation radius.

\subsubsection{Baroclinic instabilities and their effect on thermal structure}

Planets experience meridional gradients in the net radiative heating rate. At low obliquities, this gradient corresponds to net heating at the equator and net cooling at the poles, leading to meridional temperature contrasts with, generally, a hot equator and cold poles. Even if individual air columns are convectively stable (i.e., if the potential temperature increases with height in the troposphere), potential energy can be extracted from the system if the cold polar air moves downward and equatorward and if the warm equatorial air moves upward and poleward. The question is then whether dynamical mechanisms actually exist to extract this energy and thereby transport heat from the equator to the poles. In the tropics, the dominant meridional heat-transport mechanism is a thermally direct Hadley circulation (see Figure 2 and Section 2.2.1), but such circulations tend to be suppressed by planetary rotation in the extratropics. At $R o \ll 1$, the meridional temperature gradients are associated with an upward-increasing zonal wind in thermal-wind balance (Equation 2). It is useful to think about the limit where longitudinal gradients of heating and temperature are negligible - in which case the (geostrophic) wind is purely zonal-and where the meridional wind is zero, as occurs when these zonal winds are perfectly balanced. In such a hypothetical solution, the temperature at each latitude would be in local radiative equilibrium, and no meridional heat transport would occur.

It turns out that this hypothetical steady state is not dynamically stable: small perturbations on this steady solution grow over time, producing eddies that extract potential energy from the horizontal temperature contrast (transporting warm low-latitude air upward and poleward, transporting cool high-latitude air downward and equatorward, thereby lowering the center of mass, flattening isentropes.$^{7}$ and reducing the meridional temperature gradient). This is baroclinic instability, so named because it depends on the baroclinicity of the flow-i.e., on the fact that surfaces of constant density incline significantly with respect to surfaces of constant pressure. The instabilities are inherently

\footnotetext{
${ }^{7}$ Isentropes are surfaces of constant entropy, which are equivalent to surfaces of constant potential temperature. In a stably stratified atmosphere where entropy increases with altitude, isentropes will bow downward (upward) in regions that, as measured on an isobar, are hot (cold).
} 

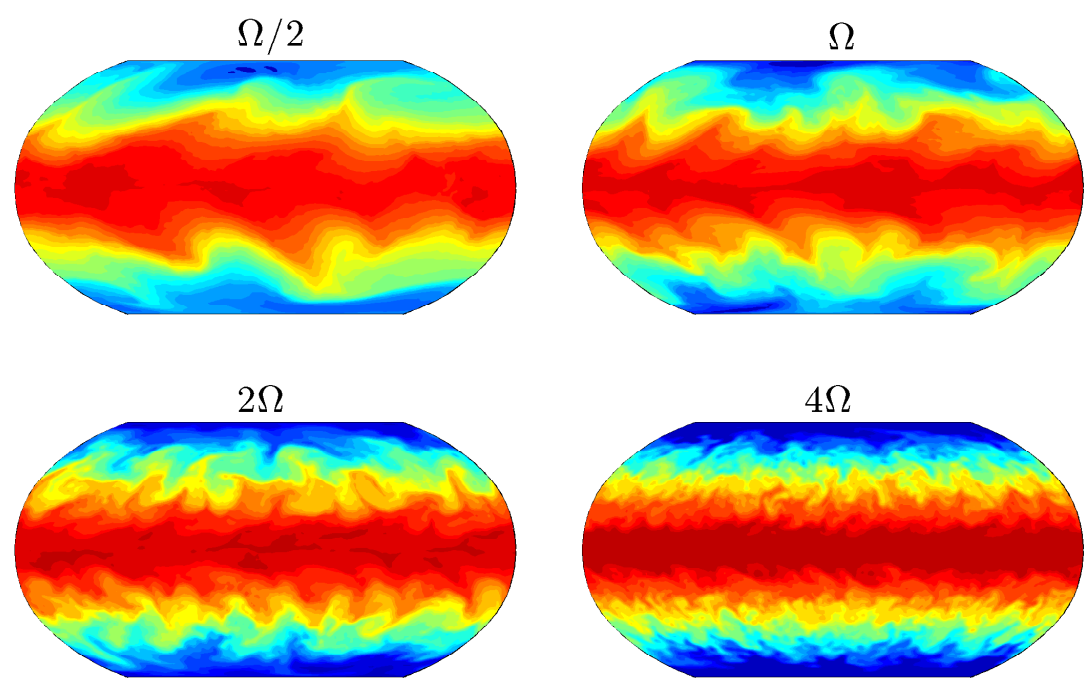

$\begin{array}{llllll}250 & 260 & 270 & 280 & 290 & 300\end{array}$

Fig. 3.- Surface temperature (colorscale, in K) from GCM experiments in Kaspi and Showman (2012), illustrating the dependence of temperature and jet structure on rotation rate. Experiments are performed using the Flexible Modeling System (FMS) model analogous to those in Frierson et al. (2006) and Kaspi and Schneider (2011); radiative transfer is represented by a two-stream, grey scheme with no diurnal cycle (i.e., the incident stellar flux depends on latitude but not longitude). A hydrological cycle is included with a slab ocean. Planetary radius, gravity, atmospheric mass, incident stellar flux, and atmospheric thermodynamic properties are the same as on Earth; models are performed with rotation rates from half (upper left) to four times that of Earth (lower right). Baroclinic instabilities dominate the dynamics in mid- and high-latitudes, leading to baroclinic eddies whose length scales decrease with increasing planetary rotation rate.

three-dimensional and manifest locally as tongues of cold and warm air penetrating equatorward and poleward in the extratropics. Figure 2 illustrates such eddies schematically and Figure 3 provides examples from GCM experiments under Earth-like conditions. The fastest-growing modes have zonal wavelengths comparable to the deformation radius and growth rates proportional to $(f / N) \partial u / \partial z$, where $\partial u / \partial z$ is the vertical shear of the zonal wind that exists in thermal wind balance with the meridional temperature gradient. For Earth-like conditions, these imply length scales of $\sim 4000 \mathrm{~km}$ and growth timescales of $\sim 3-5$ days for the dominant modes (for reviews, see, e.g., Pierrehumbert and Swanson 1995; Vallis 2006, Chapter 6).

As expected from baroclinic instability theory, the dominant length scale of the baroclinic, heat-transporting eddies in the extratropics scales inversely with the planetary rotation rate. This is illustrated in Figure 3, which shows instantaneous snapshots of the temperature in idealized Earth-like GCM experiments where the rotation rate is varied between half and four times that of Earth (Kaspi and Showman 2012). The smaller eddies in the rapidly rotating models are less efficient at transporting energy meridionally, leading to a greater equator-to-pole temperature difference in those cases. A similar dependence has been found by other authors as well (e.g. Schneider and Walker 2006, Kaspi and Schneider 2011).

In the extratropics of Earth and Mars, baroclinic insta- bilities play a key role in controlling the thermal structure, including the equator-to-pole temperature gradient and the vertical stratification (i.e., the Brunt-Vaisala frequency); this is also likely to be true in the extratropics of terrestrial exoplanets. In particular, GCM experiments suggest that the extratropics-when dominated by baroclinic instability - adjust to a dynamic equilibrium in which meridional temperature gradients and tropospheric stratifications scale together (atmospheres with larger tropospheric stratification exhibit larger meridional temperature gradients and vice versa). Figure 4 illustrates this phenomenon from a sequence of idealized GCM experiments from Schneider and Walker (2006) for terrestrial planets forced by equator-to-pole heating gradients. The abscissa shows the meridional temperature difference across the baroclinic zone and the ordinate shows the vertical stratification through the troposphere; each symbol represents the equilibrated state of a particular model including the effects of dynamics. Although the radiative-equilibrium thermal structures fill a significant fraction of the parameter space, the baroclinic eddy entropy fluxes adjust the thermal structure to a state where the vertical stratification and meridional temperature difference are comparable, corresponding to a line with a slope of one in Figure 4. The extent to which this relationship holds in general and parameter regimes where it may break down are under investigation (Zurita-Gotor and Vallis 2009, Jansen and Ferrari 
2012).

Most work on this problem has so far investigated planets forced only by equator-pole heating gradients (e.g., Schneider and Walker 2006, Kaspi and Showman 2012), but the zonal (day-night) heating gradients could significantly influence the way that baroclinic eddies regulate the thermal structure, particularly on synchronously rotating planets. Edson et al. (2011) compared GCM simulations for a control, Earth-like simulation forced only by an equator-pole heating gradient and an otherwise identical synchronously rotating planet with day-night thermal forcing. They found that the (zonal-mean) meridional isentrope slopes were gentler in the model with day-night forcing than in the control model. This differing behavior presumably results from the fact that the synchronously rotating model exhibits meridional heat fluxes that are primarily confined to the dayside (rather than occurring at all longitudes as in the control model) and from the fact that the day-night forcing generates planetary-scale standing waves (see Section 2.2.3 in the synchronous model that are absent in the control model. Both traits can influence the (zonal-mean) meridional heat transport and therefore the isentrope slopes and zonal-mean temperature differences between the equator and poles. Additional work quantifying the behavior for tidally locked planets over a wider parameter regime would be highly beneficial.

Over the past several decades, many authors have attempted to elucidate theoretically how the meridional heat fluxes due to baroclinic eddies depend on the background meridional temperature gradient, tropospheric stratification, planetary rotation rate, and other parameters. Almost all of this work has emphasized planets in an Earthlike regime, forced by equator-pole heating gradients (e.g., Green (1970), Stone (1972), Larichev and Held (1995), Held and Larichev (1996), Pavan and Held (1996), Haine and Marshall (1998), Barry et al. (2002), Thompson and Young (2006). Thompson and Young (2007), Schneider and Walker (2008), Zurita-Gotor and Vallis (2009); for reviews, see Held (1999a), and Showman et al. (2010)). If a theory for this dependence could be developed, it would constitute a major step toward a predictive theory for the dependence of the equator-to-pole temperature difference on planetary parameters. However, this is a challenging problem, and no broad consensus has yet emerged.

Nevertheless, recent GCM studies demonstrate how the equator-to-pole temperature differences and other aspects of the dynamics depend on planetary radius, gravity, rotation rate, atmospheric mass, and incident stellar flux (Kaspi and Showman 2012). These authors performed idealized GCM experiments of planets forced by equator-to-pole heating gradients, including a hydrological cycle and representing the radiative transfer using a two-stream, grey approach. Kaspi and Showman (2012) found that the equatorto-pole temperature difference decreases with increasing atmospheric mass and increases with increasing rotation rate, planetary radius (at constant interior density) or planetary density (at constant interior mass). Figure 5 shows the zonal-mean surface temperature and meridional eddy energy fluxes versus latitude for the cases with differing rotation rates (left) and atmospheric masses (right). Meridional eddy energy fluxes are weaker at faster rotation rates (Figure 5 a), presumably because the smaller eddy length scales (Figure 3) lead to less efficient energy transport. This helps to explain the fact that larger equator-to-pole temperature differences occur in the faster rotating simulations, as is evident in Figure $5 \mathrm{k}$ and Figure 3 Likewise, massive atmospheres exhibit a greater thermal storage capacity than less massive atmospheres, allowing a greater meridional energy flux at a given baroclinic eddy amplitude. Everything else being equal, planets with greater atmospheric mass therefore exhibit smaller equator-to-pole temperature differences than planets with lesser atmospheric mass (Figure 5d). Additional detailed work is warranted to clarify the physical processes controlling these trends and to seek a predictive theory for the dependences.

\subsubsection{Rossby waves}

Much of the structure of the large-scale atmospheric circulation can be understood in terms of the interaction of Rossby waves with the background flow; they are the most important wave type for the large-scale circulation. In this section we survey their linear dynamics, and follow in subsequent sections with discussions of how they help to shape the structure of the extratropical circulation via nonlinear interactions.

Rossby waves are best understood through conservation of potential vorticity (PV), which for a shallow fluid layer of thickness $h$ can be written as

$$
q=\frac{\zeta+f}{h}
$$

where $\zeta=\mathbf{k} \cdot \nabla \times \mathbf{v}$ is the relative vorticity, $\mathbf{k}$ is the vertical (upward) unit vector, and $\mathbf{v}$ is the horizontal velocity vector. As written, this is the conserved form of PV for the shallow-water equations (e.g. Pedlosky 1987, Vallis 2006 . Showman et al. 2010), but the same form holds in the threedimensional primitive equations if the relative vorticity is evaluated at constant potential temperature and $h$ is appropriately defined in terms of the gradient of pressure with respect to potential temperature (Vallis 2006, p. 187-188). To illuminate the dynamics, we consider the simplest system that supports Rossby waves, namely a one-layer barotropic model governing two-dimensional, non-divergent flow. For this case, $h$ can be considered constant, leading to conservation of absolute vorticity, $\zeta+f$, following the flow. This can be written, adopting Cartesian geometry, as

$$
\frac{\partial \zeta}{\partial t}+\mathbf{v} \cdot \nabla \zeta+v \beta=F
$$

where $v$ is the meridional velocity, $\beta \equiv d f / d y$ is the gradient of the Coriolis parameter $f$ with northward distance $y$, and $F$ represents any sources or sinks of potential vorticity. For a brief review of equation sets used in atmospheric 


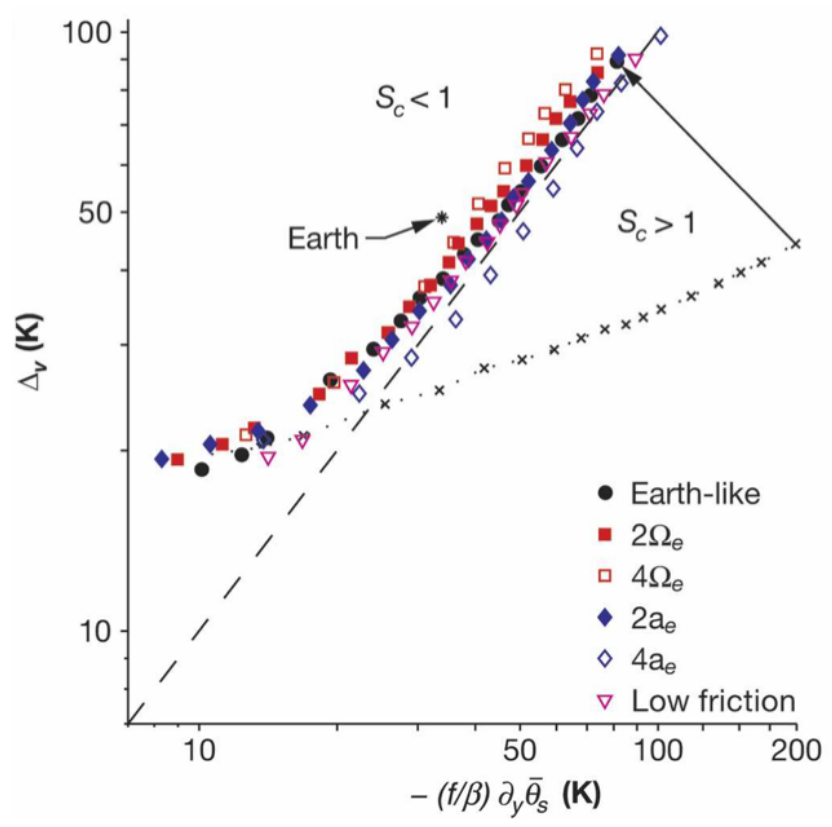

Fig. 4.- A measure of the near-surface meridional potential temperature difference across the extratropics (abscissa) and potential temperature difference in the extratropics taken vertically across the troposphere (ordinate) in models of planets forced by equator-to-pole heating gradients from Schneider and Walker (2006). When the equator-to-pole forcing is sufficiently great, the extratropical dynamics are dominated by baroclinic eddies, which adjust the thermal structure to a state where horizontal and vertical potential temperature differences in the extratropics are comparable. Different symbols denote models with differing rotation rates, planetary radii, and/or boundary-layer friction, coded in the legend. Crosses depict the radiative-equilibrium states for the Earth-like models. The dashed line denotes a slope of 1 .

dynamics, see Showman et al. (2010); more detailed treatments can be found in Vallis (2006), McWilliams (2006), or Holton (2004).

To investigate how the $\beta$ effect allows wave propagation, we consider the version of Eq. (7) linearized about a state of no zonal-mean flow, which amounts to dropping the term involving advection of $\zeta$. Given the assumption that the flow is horizontally nondivergent, we can define a streamfunction, $\psi$, such that $u=-\frac{\partial \psi}{\partial y}$ and $v=\frac{\partial \psi}{\partial x}$. This definition implies that $\zeta=\nabla^{2} \psi$, allowing the linearized equation to be written

$$
\frac{\partial \nabla^{2} \psi}{\partial t}+\beta \frac{\partial \psi}{\partial x}=0
$$

This is useful because it is now an equation in only one variable, $\psi$, which can easily be solved to determine wave behavior. Assuming that $\beta$ is constant (an approximation known as the "beta plane"), and adopting plane-wave solutions $\psi=\psi_{0} \exp [i(k x+l y-\omega t)]$, we obtain a dispersion relation 8

$$
\omega=-\frac{\beta k}{k^{2}+l^{2}}
$$

where $k$ and $l$ are the zonal and meridional wavenumbers (just $2 \pi$ over the longitudinal and latitudinal wavelengths,

${ }^{8}$ For introductions to wave equations and dispersion relations, see for example Vallis 2006), Holton (2004), Pedlosky (1987), or Pedlosky (2003). respectively) and $\omega$ is the oscillation frequency. The resulting waves are called Rossby waves. They are large-scale (wavelengths commonly $\sim 10^{3} \mathrm{~km}$ or more), low-frequency (periods of order a day or longer), and-away from the equator on a rapidly rotating planet-are in approximate geostrophic balance. As demonstrated by Equation (9), they exhibit westward phase speeds.

The wave-induced zonal and meridional velocities are defined, respectively, as $u^{\prime}=u_{0}^{\prime} \exp [i(k x+l y-\omega t)]$ and $v^{\prime}=v_{0}^{\prime} \exp [i(k x+l y-\omega t)]$, where $u_{0}^{\prime}$ and $v_{0}^{\prime}$ are complex amplitudes. The above solution implies that

$$
u_{0}^{\prime}=-i l \psi_{0} \quad v_{0}^{\prime}=i k \psi_{0} .
$$

The velocities represented by these relations are parallel to lines of constant phase and therefore perpendicular to the direction of phase propagation. Equation (10) implies that the zonal and meridional wave velocities are correlated; this provides a mechanism by which Rossby wave generation, propagation, and dissipation can transport momentum and thereby modify the mean flow, a point we return to in Section 2.1.4.

Physically, the restoring force for Rossby waves is the $\beta$ effect, namely, the variation with latitude of the planetary vorticity. Because the Coriolis parameter varies with latitude, PV conservation requires that any change in latitude of fluid parcels will cause a change in the relative vorticity. The flow associated with these relative vorticity pertur- 

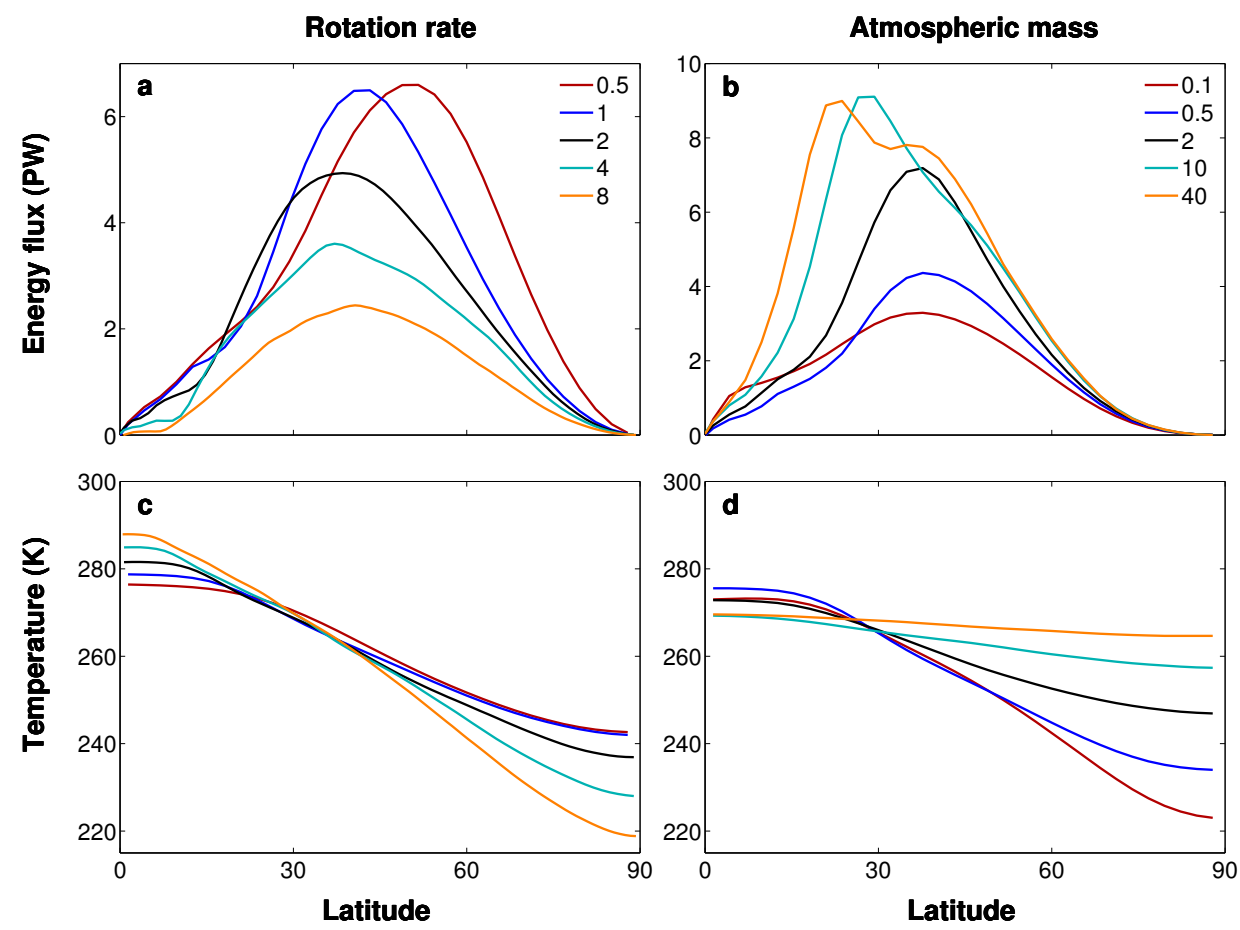

Fig. 5.- Latitude dependence of the vertically and zonally integrated meridional energy flux (a and b) and vertically and zonally averaged temperature ( $\mathrm{c}$ and d) in GCM experiments from Kaspi and Showman (2012) for models varying rotation rate and atmospheric mass. The energy flux shown in (a) and (b) is the flux of moist static energy, defined as $c_{p} T+g z+L q$, where $c_{p}$ is specific heat at constant pressure, $g$ is gravity, $z$ is height, $L$ is latent heat of vaporization of water, and $q$ is the water vapor abundance. The left column (a and c) explores sensitivity to rotation rate; models are shown with rotation rates ranging from half to eight times that of Earth. In these experiments, the atmospheric mass is held constant at the mass of Earth's atmosphere. Right column ( $b$ and d) explores the sensitivity to atmospheric mass; models are shown with atmospheric masses from 0.1 to 40 times the mass of Earth's atmosphere. In these experiments, the rotation rate is set to that of Earth. The equator-to-pole temperature difference is smaller, and the meridional energy flux is larger, when the planetary rotation rate is slower, and/or when the atmospheric mass is larger. Other model parameters, including incident stellar flux, optical depth of the atmosphere in the visible and infrared, planetary radius, and gravity, are Earth-like and are held fixed in all models. 
bations leads to oscillations in the position of fluid parcels and thereby allows wave propagation. The meridional velocities deform the phase surfaces in a manner that leads to the westward phase velocities captured in Equation 9 ) (see discussion in Vallis (2006) or Holton (2004)).

\subsubsection{Jet formation I: Basic mechanisms}

A wide range of numerical experiments and observations show that zonal jets tend to emerge spontaneously on rapidly rotating planets. On many planets, such zonal jets dominate the circulation, and thus understanding them is crucial to understanding the circulation as a whole. The dynamics controlling jets is intimately connected to the dynamics controlling the meridional temperature gradients, vertical stratification, and other aspects of the dynamics. Sufficiently strong jets exhibit sharp gradients of the meridional PV gradient, and as such they can act as barriers to the meridional mixing of heat, moisture, and chemical tracers (e.g., Beron-Vera et al. 2008), significantly affecting the meridional structure of the atmosphere.

While there exist many mechanisms of jet formation, among the most important is the interaction of Rossby waves with the mean flow. This mechanism plays a key role in causing the extratropical jets on Earth, Mars, and perhaps Jupiter and Saturn; the mechanism is similarly expected to play an important role in the atmospheres of terrestrial exoplanets.

As Thompson (1971), Held (1975), and many subsequent authors have emphasized, a key property of meridionally propagating Rossby waves is that they induce a meridional flux of prograde (eastward) eddy angular momentum into their generation latitude. To illustrate, we again consider the solutions to Eq. (8). The latitudinal transport of (relative) eastward eddy momentum per mass is $\overline{u^{\prime} v^{\prime}}$, where $u^{\prime}$ and $v^{\prime}$ are the deviation of the zonal and meridional winds from their zonal mean, and the overbar denotes a zonal average. Using Eq. (10) shows that

$$
\overline{u^{\prime} v^{\prime}}=-\frac{1}{2} \hat{\psi}^{2} k l \text {. }
$$

Now, the dispersion relation (9) implies that the meridional group velocity is $\partial \omega / \partial l=2 \beta k l /\left(k^{2}+l^{2}\right)^{2}$. Since the group velocity must point away from the region where the Rossby waves are generated, we must have $k l>0$ north of this wave source and $k l<0$ south of the wave source. Combining this information with Eq. (11) shows that $\overline{u^{\prime} v^{\prime}}<0$ north of the source and $\overline{u^{\prime} v^{\prime}}>0$ south of the source. Thus, the Rossby waves flux eastward momentum into the latitude of the wave source.

This process is illustrated in Fig. 6. Suppose some process generates Rossby wave packets at a specific latitude, which propagate north and south from the latitude of generation. The northward propagating wave packet exhibits eddy velocities tilting northwest-southeast, while the southward propagating packet exhibits eddy velocities tilting southwest-northeast. The resulting eddy velocities visusally resemble an eastward-pointing chevron pattern cen- tered at the latitude of wave generation. The correlation between these velocities leads to non-zero Reynolds stresses (that is, a non-zero $\overline{u^{\prime} v^{\prime}}$ ) and a flux of angular momentum into the wave generation latitude. An example of this schematic chevron pattern in an actual GCM experiment is shown in Figure 7

The above reasoning is for free (unforced) waves but can be extended to an atmosphere forced by vorticity sources/sinks and damped by frictional drag (see reviews in Held 2000, Vallis 2006; Showman and Polvani 2011). In the nondivergent, barotropic system, the zonal-mean zonal momentum equation is given by, adopting a Cartesian coordinate system for simplicity,

$$
\frac{\partial \bar{u}}{\partial t}=-\frac{\partial\left(\overline{u^{\prime} v^{\prime}}\right)}{\partial y}-\frac{\bar{u}}{\tau_{\text {drag }}}
$$

where we have decomposed the winds into their zonal means (given by overbars) and the deviations therefrom (given by primes), such that $u=\bar{u}+u^{\prime}, v=\bar{v}+v^{\prime}$, $\zeta=\bar{\zeta}+\zeta^{\prime}$, etc. Here, $y$ is northward distance and we have parameterized drag by a linear (Rayleigh) friction that relaxes the winds toward zero over a specified drag time constant $\tau_{\text {drag. }}$. To link the momentum budget to the vorticity sources and sinks, we first note that, for the horizontally nondivergent system, the definition of vorticity implies that $\overline{v^{\prime} \zeta^{\prime}}=-\partial\left(\overline{u^{\prime} v^{\prime}}\right) / \partial y$. Second, we multiply the linearized version of Eq. (7) by $\zeta^{\prime}$ and zonally average, yielding an equation for the so-called pseudomomentum

$$
\frac{\partial \mathcal{A}}{\partial t}+\overline{v^{\prime} \zeta^{\prime}}=\frac{\overline{\zeta^{\prime} F^{\prime}}}{2\left(\beta-\frac{\partial^{2} \bar{u}}{\partial y^{2}}\right)}
$$

where $\mathcal{A}=\left(\beta-\partial^{2} \bar{u} / \partial y^{2}\right)^{-1} \overline{\zeta^{\prime 2}} / 2$ is the pseudomomentum, which characterizes the amplitude of the eddies in a statistical (zonal-mean) sense. Here, $F^{\prime}$ is the eddy component of the vorticity source/sink defined in Equation (7). We then combine Eqs. (12) and (13). If the zonal-mean flow equilibrates to a statistical steady state (i.e., if $\partial \bar{u} / \partial t$ and $\partial \mathcal{A} / \partial t$ are zero), this yields a relationship between the zonal-mean zonal wind, $\bar{u}$, and the eddy generation of vorticity:

$$
\frac{\bar{u}}{\tau_{\text {drag }}}=\frac{\overline{\zeta^{\prime} F^{\prime}}}{2\left(\beta-\frac{\partial^{2} \bar{u}}{\partial y^{2}}\right)} .
$$

(Note that this equilibration does not require the eddies themselves to be steady, but rather simply that their zonally averaged mean amplitude, characterized by $\mathcal{A}$, is steady.)

What are the implications of this equation? Consider a region away from wave sources where the eddies are dissipated. Dissipation acts to reduce the eddy amplitudes, implying that $F^{\prime}$ and $\zeta^{\prime}$ exhibit opposite signs (see Eq. 7). 9 Therefore, regions of net wave dissipation will exhibit $\zeta^{\prime} F^{\prime}<0$ and the zonal-mean zonal wind will be west-

\footnotetext{
${ }^{9}$ For example, for the specific case of linear drag, represented in the zonal and meridional momentum equations as $-u / \tau_{\text {drag }}$ and $-v / \tau_{\text {drag }}$, respectively, then in the absence of forcing $F=-\zeta / \tau_{\text {drag }}$.
} 

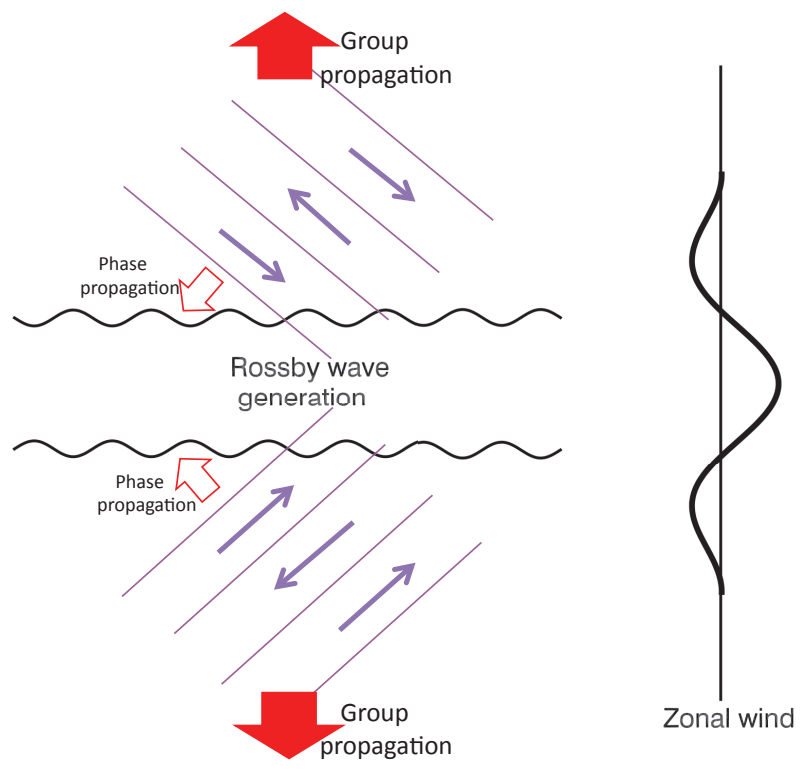

Fig. 6.- Schematic illustration of how Rossby wave generation can lead to the formation of zonal jets. Imagine that some process - such as baroclinic instability — generates Rossby waves at a specific latitude, and that the Rossby waves then propagate north and south from their latitude of generation. Rossby waves with northward group propagation exhibit eddy velocities tilting northwest-to-southeast, whereas those with southward group propagation exhibit eddy velocities tilting southwest-to-northeast. These correlations imply that the waves transport eastward eddy momentum into the latitude region where Rossby waves are generated, resulting in formation of an eddy-driven jet.

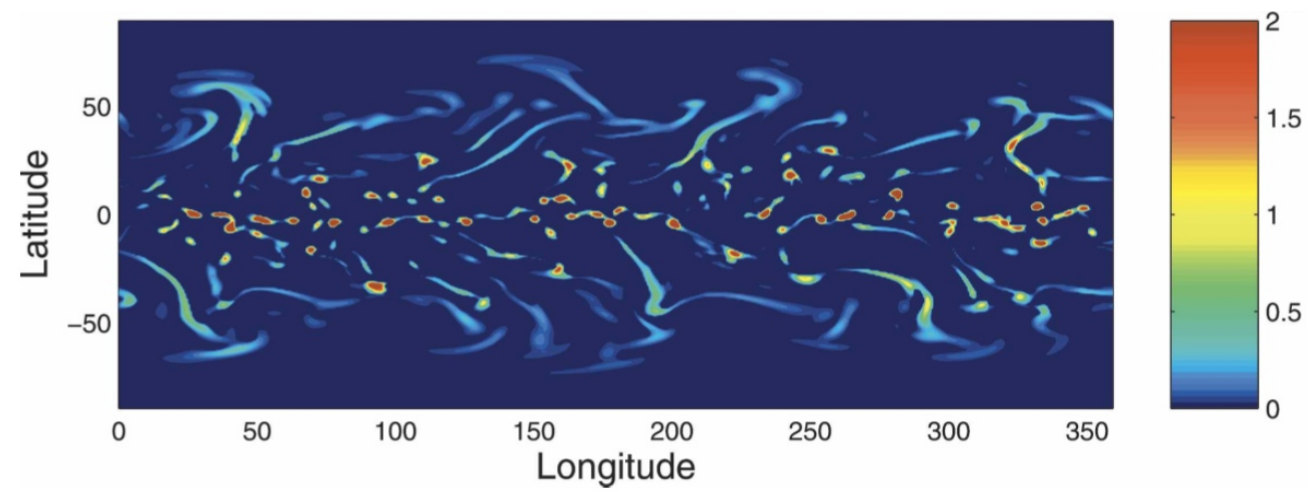

Fig. 7.- Instantaneous precipitation (units $10^{-3} \mathrm{~kg} \mathrm{~m}^{-2} \mathrm{~s}^{-1}$ ) in an idealized Earth GCM by Frierson et al. (2006), illustrating the generation of phase tilts by Rossby waves as depicted schematically in Figure 6 Baroclinic instabilities in midlatitudes $\left(\sim 40-50^{\circ}\right)$ generate Rossby waves that propagate meridionally. On the equatorward side of the baroclinically unstable zone (latitudes of $\sim 20-50^{\circ}$ ), the waves propagate equatorward, leading to characteristic precipitation patterns tilting southwest-northeast in the northern hemisphere and northwest-southeast in the southern hemisphere. In contrast, the phase tilts are reversed (though less well organized) poleward of $\sim 50-60^{\circ}$ latitude, indicative of poleward Rossby wave propagation. Equatorward of $20^{\circ}$ latitude, tropical convection dominates the precipitation pattern. 
ward. On the other hand, in region where eddies are generated, the wave sources act to increase the wave amplitudes, implying that $F^{\prime}$ and $\zeta^{\prime}$ exhibit the same signs. In such a region, $\overline{\zeta^{\prime} F^{\prime}}>0$ and the zonal-mean zonal wind will be eastward. The two regions are linked because waves propagate (in the sense of their group velocity) from their latitude of generation to their latitude of dissipation; this allows a statistical steady state in both the zonal-mean eddy amplitudes and the zonal-mean zonal wind to be achieved despite the tendency of damping (forcing) to locally decrease (increase) the eddy amplitudes. In summary, we thus recover the result that eastward net flow occurs in regions of net wave generation while westward net flow occurs in regions of net wave damping.

In the extratropics, baroclinic instabilities constitute a primary mechanism for generating Rossby waves in the free troposphere, which propagate from the latitude of instability to surrounding latitudes (see Figure 7). To the extent that the extratropics of exoplanets are baroclinically unstable, this mechanism thus implies the emergence of the extratropical eastward jets; indeed, the midlatitude jets in the tropospheres of Earth and Mars result from this process. Such "eddy-driven" jets are illustrated schematically in Figure $22^{10}$ To be more precise, the eddy-momentum flux convegence should be thought of as driving the surface wind (e.g., Held 2000). The only torque that can balance the vertically integrated eddy-momentum flux convergence-and the associated eastward acceleration-is frictional drag at the surface. Thus, in latitudes where baroclinic instability or other processes leads to strong Rossby wave generation, and the radiation of those waves to other latitudes, eastward zonal-mean winds emerge at the surface. The zonal wind in the upper troposphere is then set by the sum of the surface wind and the vertically integrated wind shear, which is in thermal-wind balance with the meridional temperature gradients.

In the above theory, the only source of explicit damping was a linear drag, but in the real extratropical atmosphere, wave breaking will play a key role and can help explain many aspects of extratropical jets. Indeed, much of jet dynamics can be understood in terms of spatially inhomogeneous mixing of potential vorticity (PV, see Equation 6) caused by this wave breaking (Dritschel and McIntyre 2008). Rossby waves manifest as meridional undulations in PV contours, and Rossby wave breaking implies that the PV contours become so deformed that they curl up and overturn in the longitude-latitude plane (see Figure 8 for an example). Such overturning generally requires large wave amplitudes; in particular, the local, wave-induced perturbation to the meridional PV gradient must become comparable to the background (zonal-mean) gradient, so that, locally, the meridional PV gradient changes sign. This criterion implies that Rossby wave breaking occurs more

\footnotetext{
${ }^{10}$ In the terrestrial literature, these are referred to as "eddy-driven" jets to distinguish them from the subtropical jets that occur at the poleward edge of the Hadley cell (see $\$ 2.2$.
}

easily (i.e., at smaller wave amplitude) in regions of weak PV gradient than in regions of strong PV gradient. Now, the relationship between PV and winds implies that eastward zonal jets comprise regions where the (zonal-mean) meridional PV gradient is large, whereas westward zonal jets comprise regions where the meridional PV gradient is small (Figure 9). Therefore, vertically and meridionally propagating Rossby waves are more likely to break between (or on the flanks of) eastward jets, where the PV gradient is small, than at the cores of eastward jets, where the PV gradient is large. This wave breaking causes irreversible mixing and homogenization of PV. The mixing is thus spatially inhomogeneous-mixing preferentially homogenizes the PV in the regions where its gradient is already weak, and sharpens the gradients in between. As emphasized by Dritschel and McIntyre (2008), this is a positive feedback: by modifying the background PV gradient, such mixing promotes continued mixing at westward jets but inhibits future mixing at eastward jets. Because the PV jumps are associated with eastward jets, this process tends to sharpen eastward jets and leads to broad "surf zones" of westward flow in between. This is precisely the behavior evident in Figure 8. Because of this positive feedback, one generally expects that when an initially homogeneous system is stirred, robust zonal jets should spontaneously emergeeven if the stirring itself is not spatially organized (e.g., Dritschel and McIntyre 2008, Dritschel and Scott 2011, Scott and Dritschel 2012; Scott and Tissier 2012). Nevertheless, strong radiative forcing and/or frictional damping represent sources and sinks of PV that can prevent a pure PV staircase (and the associated zonal jets) from being achieved. The factors that determine the equilibrium PV distribution in such forced/damped cases is an area of ongoing research.

\subsubsection{Jet formation II: Rossby wave interactions with tur- bulence}

Additional insights on the formation of zonal jets emerge from a consideration of atmospheric turbulence. In the $R o \ll 1$ regime, the interaction of large-scale atmospheric turbulence with planetary rotation - and in particular with the $\beta$ effect-generally leads to the formation of a zonally banded appearance and the existence of zonal jets (for important examples, see Rhines (1975), Williams (1978), Maltrud and Vallis (1993), Cho and Polvani (1996), Huang and Robinson (1998), and Sukoriansky et al. (2007); reviews can be found in Vasavada and Showman (2005), Showman et al. (2010), and Vallis (2006 chapter 9)). By allowing Rossby waves, the $\beta$ effect introduces a fundamental anisotropy between the zonal and meridional direction that often leads to jets.

Consider the magnitudes of the terms in the vorticity equation (7). Being a curl of the wind field, the relative vorticity has characteristic magnitude $\frac{U}{L}$. Therefore, the advection term $\mathbf{v} \cdot \nabla \zeta$ has characteristic magnitude $\frac{U^{2}}{L^{2}}$, where $L$ is some horizontal lengthscale of interest. The $\beta$ term 

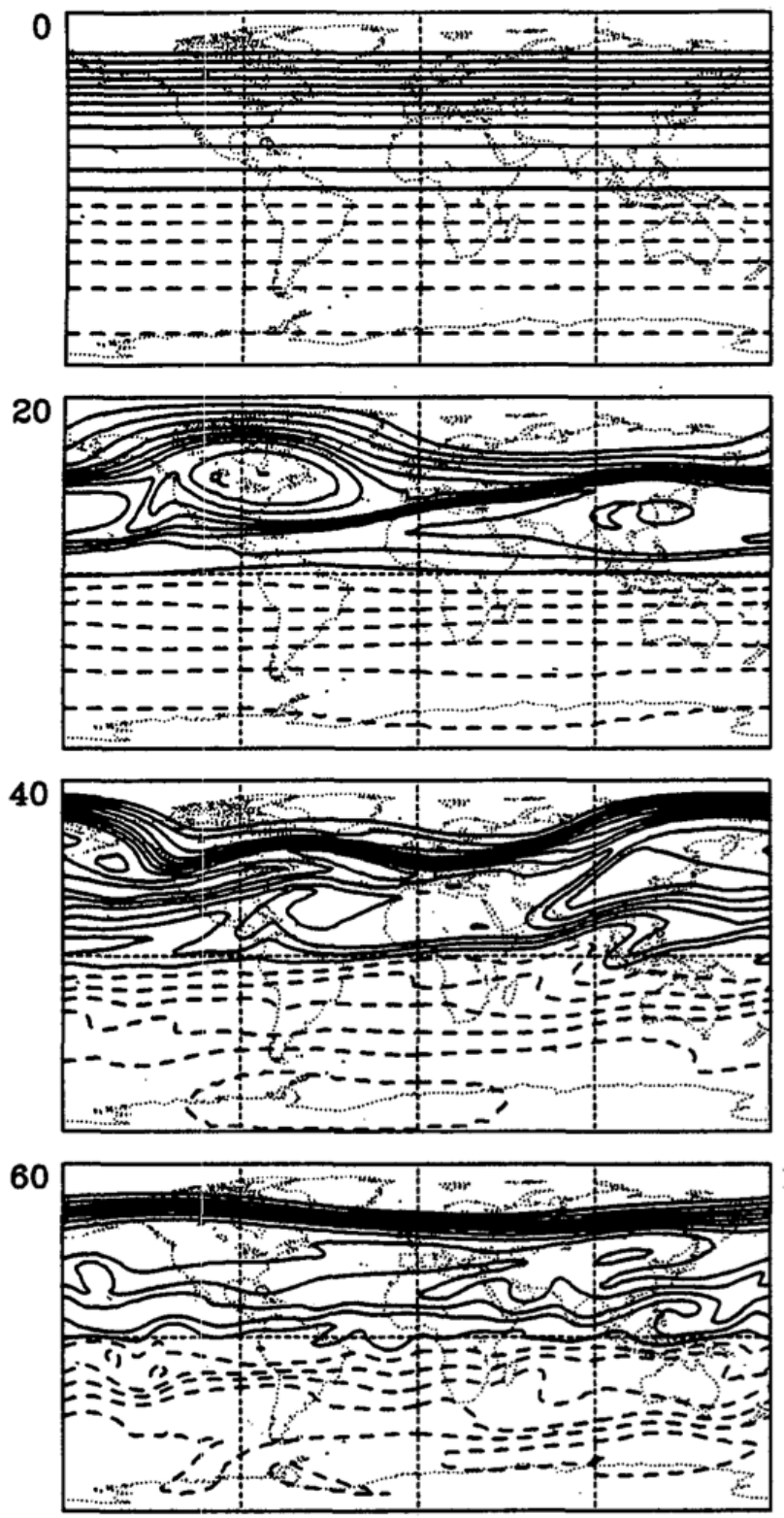

Fig. 8. - Demonstration of how breaking Rossby waves can shape jet dynamics. Plots show time evolution of the potential vorticity (in contours, with positive solid, negative dashed, and a contour interval of $0.25 \times 10^{-8} \mathrm{~m}^{-1} \mathrm{~s}^{-1}$ ) in a shallowwater calculation of Earth's stratospheric polar vortex by Polvani et al. (1995). The model is initialized from a zonallysymmetric initial condition with broadly distributed PV organized into a polar vortex centered over the north pole (top panel). A topographic perturbation is then introduced to generate a Rossby wave, which manifests as undulations in the PV contours (second panel). The wave amplitude becomes large enough for the undulations to curl up, leading to wave breaking (second and third panels). The edge of the vortex (corresponding to the region of tighly spaced PV contours) is resistant to such wave breaking, but the regions of weaker meridional PV gradient on either side are more susceptible. This breaking mixes and homogenizes the PV in those regions, thereby lessening the meridional gradient still further (this manifests as a widening in the latitudinal separation of the PV contours, especially from the equator to $\sim 60^{\circ} \mathrm{N}$ latitude, in the third and fourth panels). In contrast, stripping of material from the vortex edge by this mixing process causes a sharpening of the PV jump associated with the vortex edge (visible as a tightening of the contour spacing at $\sim 70-80^{\circ} \mathrm{N}$ latitude in the fourth panel). This sharp PV jump is associated with a narrow, fast eastward jet—-the polar jet—at the edge of the polar vortex. 

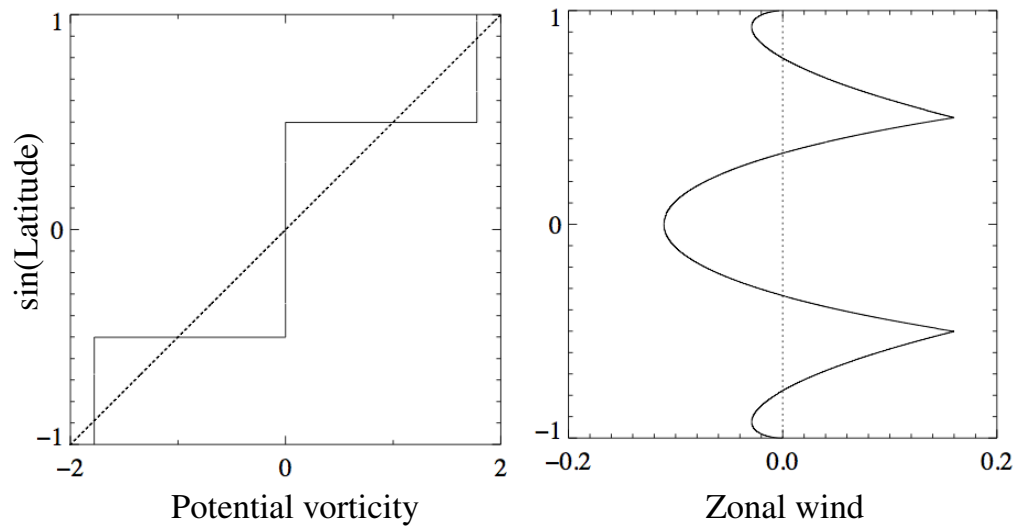

Fig. 9.- Relationship between PV (left) and zonal winds (right) versus the sine of latitude for a flow consisting of a PV "staircase" with constant-PV strips separated by regions of sharp PV gradients. In a motionless fluid on a spherical planet, the zonal wind is zero, and the PV increases smoothly with sine of latitude (dotted lines). But if PV is homogenized into strips, as shown on the left, then the implied zonal wind structure (demanded by the relationship between PV and winds) is as shown on the right (solid curves). Thus, homogenization of PV into strips on rapidly rotating planets implies the emergence of zonal jets. This is for the specific case of a 2D, horizontally non-divergent flow governed by Equation (7), but a similar relationship holds even in more realistic models: regions of weak PV gradients correspond to regions of broad westward flow, while PV discontinuities correspond to sharp eastward jets. For the specific case of a zonally symmetric flow governed by Equation (7), in Cartesian geometry with constant $\beta$, it is straightforward to show that the zonal-wind profile consists of parabolas connected end-to-end. For analytic solutions in more general cases, see Marcus and Lee (1998), Dunkerton and Scott (2008), and Wood and McIntyre (2010). From Scott (2010).

has characteristic magnitude $\beta U$. For a given wind amplitude, the advection term dominates at small scales (i.e., as $L \rightarrow 0$ ). At these scales, the $\beta$ term is unimportant, and the equation therefore describes two-dimensional turbulence. Such turbulence will be isotropic, because (at scales too small for $\beta$ to be important) the equation contains no terms that would distinguish the east-west from the north-south directions. On the other hand, at large scales, the $\beta$ term will dominate over the nonlinear advection term, and this implies that Rossby waves dominate the dynamics. The transition between these regimes occurs at the Rhines scale,

$$
L_{R}=\left(\frac{U}{\beta}\right)^{1 / 2}
$$

The Rhines scale is traditionally interpreted as giving the transition scale between the regimes of turbulence (at small scales) and Rossby waves (at large scales). Generally, two-dimensional and quasi-two-dimensional fluids exhibit an upscale energy transfer from small scales to large scales (e.g., Vallis 2006, Chapter 8). Therefore, if turbulent energy is injected into the fluid at small scales (e.g., through convection, baroclinic instabilities, or other processes), turbulent interactions can drive the energy toward larger scales where it can be affected by $\beta$. At scales close to the Rhines scale, the $\beta$ effect forces the turbulence to become anisotropic: since the term $v \beta$ involves only the meridional speed but not the zonal speed, the dynamics becomes different in the east-west and north-south directions.

This anisotropy causes the development of zonal banding in planetary atmospheres. As shown by Vallis and Mal- trud (1993) and other authors, the transition between turbulence and Rossby waves is anisotropic, occurring at different length scales for different wavevector orientations. (Equation 15 gives the characteristic value ignoring this geometric effect.) Essentially, non-linear interactions are better able to transfer turbulent energy upscale for structures that are zonally elongated than for structures that are isotropic or meridionally elongated. Preferential development of zonally elongated structures at large scales, often consisting of zonal jets, results. In many cases, the turbulent interactions do not involve the gradual transport of energy to incrementally ever-larger lengthscales (a transport that would be "local" in spectral space) but rather tend to involve spectrally non-local interactions wherein small-scale turbulence directly pumps the zonal jets (e.g., Nozawa and Yoden 1997; Huang and Robinson 1998; Sukoriansky et al. 2007; Read et al. 2007; Wordsworth et al. 2008).

These processes are illustrated in Figure 10, which shows the results of three solutions of the $2 \mathrm{D}$, non-divergent vorticity equation (7) starting from an initial condition containing numerous small-scale, close-packed, isotropic vortices. The model on the left is non-rotating, the model in the middle has intermediate rotation rate, and the model on the right is rapidly rotating. In the non-rotating case (left panel), the 2D turbulence involves vortex mergers that drive turbulent energy from small scales toward larger scales. The flow remains isotropic and no jets form. In the rapidly rotating case, however, the turbulence interacts with Rossby waves at scales comparable to $L_{R}$, leading to robust zonal banding (right panel).

A wide variety of studies have shown that zonal jets gen- 


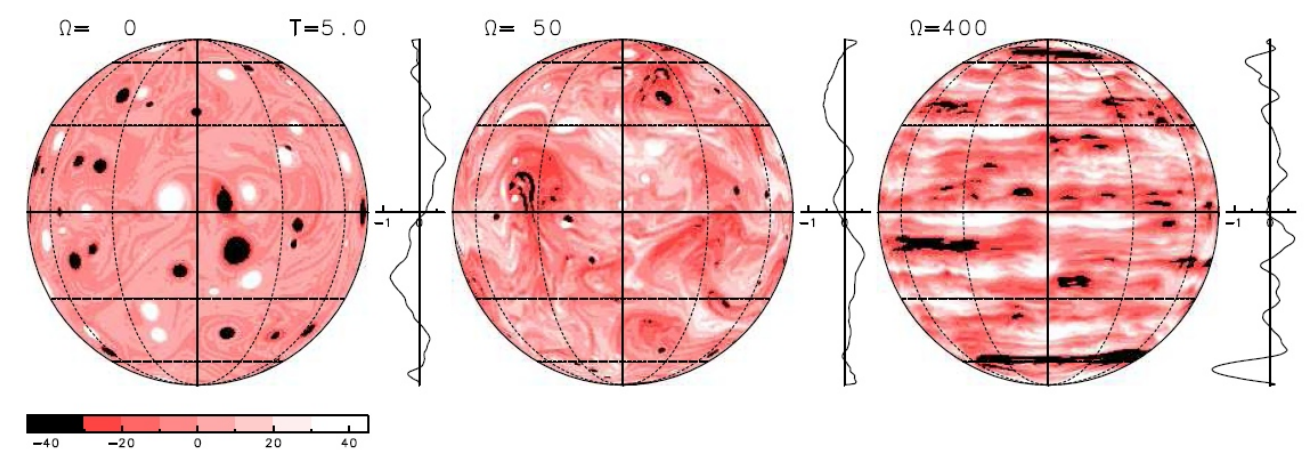

Fig. 10.- Solutions of the 2D non-divergent barotropic vorticity equation (7) illustrating the effect of planetary rotation on large-scale atmospheric turbulence. Three simulations are shown, initialized from identical initial conditions containing turbulence at small length scales. Only the rotation rate differs between the three models; from left to right, the rotation rates are zero, intermediate, and rapid. There is no forcing or large-scale damping (save for a numerical viscosity required for numerical stability) so that the total flow energy is nearly constant in all three cases. In the non-rotating case, energy cascades to larger length scales as vortices merge, but the flow remains isotropic. In the rapidly rotating case, the flow is highly anisotropic and zonal banding develops. From Hayashi et al. (2000); see also Yoden et al. (1999), Ishioka et al. (1999), and Hayashi et al. (2007).

erated in this way exhibit characteristic meridional widths comparable to the Rhines scale [for reviews, see Vasavada and Showman (2005), Vallis (2006, Chapter 9), and Del Genio et al. (2009)]. Nevertheless, there has been significant debate about the relationship of the Rhines scale to other important lengthscales, such as Rossby deformation radius. The characteristic lengthscale for baroclinic instabilities is the deformation radius; therefore, in a flow driven by baroclinic instabilities, turbulent energy is injected at scales close to $L_{D}$. In principle, it is possible that $L_{R}$ greatly exceeds $L_{D}$, in which case nonlinear interactions would transfer the energy upscale from the deformation radius to the scale of the jets themselves. However, atmospheric GCMs under Earth-like conditions have generally found that the circulation adjusts to a state where the deformation radius is comparable to the Rhines scale (e.g., Schneider and Walker 2006. 2008). In this case, baroclinic instabilties directly inject turbulent energy into the flow at scales comparable to the meridional width of the zonal jets. The injected energy interacts nonlinearly with the mean flow to generate jets, but significant upscale transfer of the energy is not necessarily involved. Still, situations exist (including in Earth's ocean) where the Rhines scale and deformation radius differ substantially and significant upscale energy transfer occurs between the two scales (e.g. Jansen and Ferrari 2012).

These arguments suggest that, for extratropical jets driven by baroclinic instability, the relative sizes of the deformation radius and of the extratropics itself determines the number of jets. When a planet is small, like Earth or Mars, only one strip of baroclinic instabilities-and one eddy-driven jet—can fit into the baroclinic zone (as illustrated schematically in Figure 2). When the planet is relatively large relative to the eddy size, however, the baroclinic zone breaks up into multiple bands of baroclinic instability, and hence multiple jet streams. Figure 11 shows an example from Schneider and Walker (2006) showing an Earth-like case (top) and a case at four times the Earth rotation rate (bottom). The Earth case exhibits only one, midlatitude eddy-driven jet in each hemisphere. In the rapidly rotating case, the deformation radius is four times smaller, and the baroclinic zone breaks up into three jets in each hemisphere. This process coincides with a steepening of the isentropes (thin grey contours), which is associated with a greater equator-to-pole temperature difference, consistent with those shown in Figures 3 and 5 .

\subsection{Tropical Regime}

The tropics, defined here as regimes of $R o \gtrsim 1$, are inherently ageostrophic, and their dynamics differ significantly from those of the extratropics. Unlike the case of geostrophic flow, horizontal temperature gradients in the $R o \gtrsim 1$ regime tend to be modest, which affects the dynamics in myriad ways.

The tendency toward weak temperature gradients can be motivated by considering arguments analogous to those leading up to Equation (4). In the absence of a significant Coriolis force, large-scale horizontal pressure-gradient forces tend to be balanced by advection, represented to order of magnitude as $U^{2} / L$. The fractional horizontal potential temperature difference is then (Charney 1963)

$$
\frac{\delta \theta_{h}}{\theta} \sim \frac{U^{2}}{g D} \sim F r \quad R o \gtrsim 1
$$

Comparison with Equation (4) immediately shows that, in the rapidly rotating regime characterized by $R o \ll 1$, the lateral temperature contrasts are a factor of $R o^{-1}$ bigger than they are in the slowly rotating regime of $R o \gtrsim 1$. These trends are evident in the GCM experiments in Figure 3 and 5 k. For a given wind speed, slowly rotating planets tend to be more horizontally isothermal than rapidly 

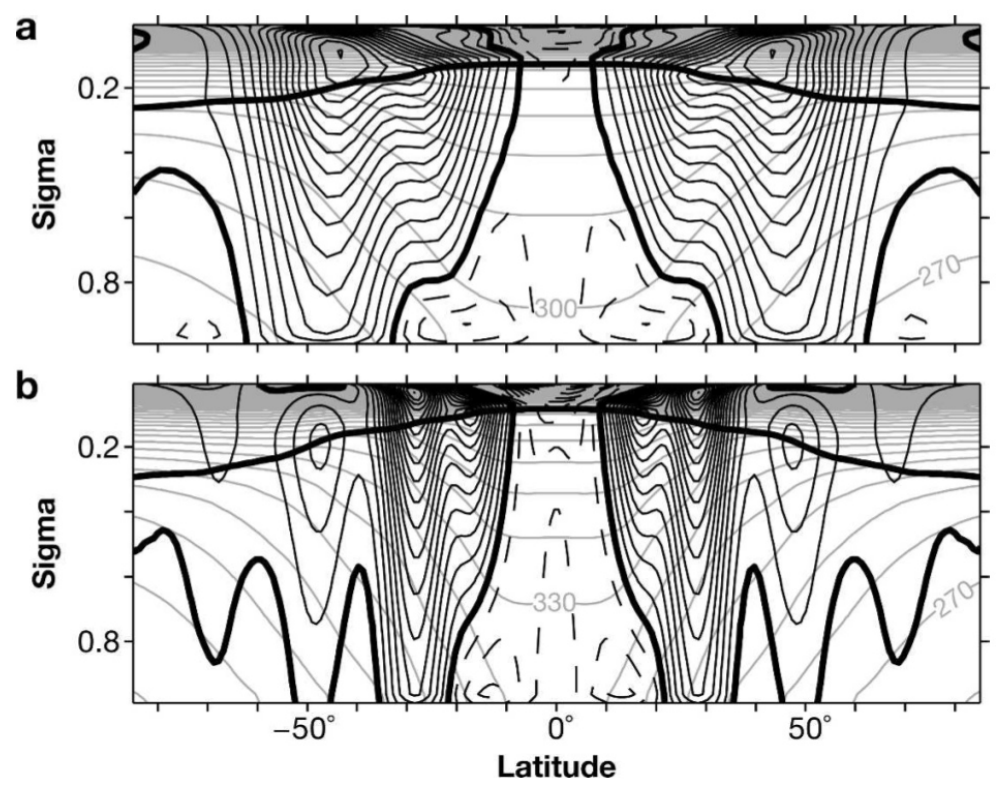

Fig. 11.- Idealized GCM simulations of Earth-like planets exhibiting jet formation. These models are forced by equatorpole heating gradients. Black contours show zonal-mean zonal wind (positive solid, negative dashed, zero contour is thick; contour interval is $2.5 \mathrm{~m} \mathrm{~s}^{-1}$ ). Grey contours show zonal-mean potential temperature ( $\mathrm{K}$, contour interval is $10 \mathrm{~K}$ ). Heavy line near top denotes the tropopause. Vertical coordinate is ratio of pressure to surface pressure. The top panel shows an Earth-like case, while the bottom panel shows a case at 4 times the Earth rotation rate. The Earth-like case exhibits one eddy-driven jet in each hemisphere. In the rapidly rotating case, this jet is confined much closer to the equator and additional, eddy-driven jets have emerged in each hemisphere. From Schneider and Walker (2006).

rotating planets. In the case of a typical terrestrial planet where $U \sim 10 \mathrm{~m} \mathrm{~s}^{-1}, D \sim 10 \mathrm{~km}$, and $g \approx 10 \mathrm{~m} \mathrm{~s}^{-2}$, we have $\mathrm{Fr} \sim 10^{-3}$. One might thus expect that, in the tropics of Earth, and globally on Venus, Titan, and slowly rotating exoplanets, the lateral temperature contrasts are $\sim 1 \mathrm{~K}$. Note, however, that because of the quadratic dependence of $\delta \theta_{h}$ on wind speed, large temperature differences could occur if the winds are sufficiently fast. Of course, additional arguments would be needed to obtain a self-consistent prediction of both $\delta \theta_{h}$ and $U$ on any given planet.

We here discuss several of the major dynamical mechanisms relevant for the tropical regime.

\subsubsection{Hadley circulation and subtropical jets}

Planets generally exhibit meridional gradients of the mean incident stellar flux, with, at low obliquity, highly irradiated, warm conditions at low latitudes and poorly irradiated, cooler conditions at high latitudes. The Hadley circulation represents the tropical response to this insolation gradient and is the primary mechanism for meridional heat transport in the tropical atmosphere. Stripped to its essence, the Hadley circulation in an atmosphere forced primarily by an equator-pole heating gradient can be idealized as an essentially two-dimensional circulation in the latitude-height plane: hot air rises near the equator, moves poleward aloft, descends at higher latitudes, and returns to the equator along the surface (see Figure 2 for a schematic). All the terrestrial planets with thick atmospheres in the so- lar system-Earth, Venus, Mars, and Titan-exhibit Hadley cells, and Hadley circulations will likewise play an important role on terrestrial exoplanets.

The Hadley circulations exert a significant effect on the mean planetary climate. Because of its meridional energy transport, the meridional temperature gradient across the Hadley circulation tends to be weak. Moreover, on planets with hydrological cycles (Section 3), the ascending branch tends to be a location of cloudiness and high rainfall, whereas the descending branches tend to be drier and more cloud-free. In this way, the Hadley circulation exerts control over regional climate. On Earth, most tropical rainforests occur near the equator, at the latitude of the ascending branch, while many of the world's major deserts (the Sahara, the American southwest, Australia, and South Africa) occur near the latitudes of the descending branches. Because the latitudinal and vertical distribution of cloudiness and humidity can significantly influence the planetary albedo and greenhouse effect, the mean planetary surface temperature-as well as the distribution of temperature, cloudiness, and humidity across the planet—depend significantly on the structure of the Hadley circulation. Moreover, by helping to control the equator-pole distribution of temperature, humidity, and clouds, the Hadley circulation will influence the conditions under which terrestrial planets can experience global-scale climate feedbacks, including transitions to globally glaciated "snowball" states, atmospheric collapse, and runaway greenhouses (Section 4 . 
The structure of the Hadley cell is strongly controlled by planetary rotation. Frictional drag acts most strongly in the surface branch of the circulation, and the upper branch, being decoupled from the surface, is generally less affected by frictional drag. A useful limit to consider is one where the upper branch is frictionless, such that individual air parcels ascending at the equator conserve their angular momentum per unit mass about the planetary rotation axis, given by $m=(\Omega a \cos \phi+u) a \cos \phi$, as they move poleward (Held and Hou 1980). If the ascending branch is at the equator and exhibits zero zonal wind, then the zonal wind in the upper (poleward-flowing) branch of such an angular-momentum conserving circulation is

$$
u=\Omega a \frac{\sin ^{2} \phi}{\cos \phi}
$$

where $a$ is the planetary radius and $\phi$ is latitude. Thus, in the upper branch of the Hadley cell, the zonal wind increases rapidly with latitude, the more so the faster the planet rotates or the larger the planetary size. Under modern Earth conditions, this equation implies that the zonal wind is $134 \mathrm{~m} \mathrm{~s}^{-1}$ at $30^{\circ}$ latitude, reaches $1000 \mathrm{~m} \mathrm{~s}^{-1}$ at a latitude of $67^{\circ}$, and becomes infinite at the poles. This is of course impossible, and implies that planetary rotation, if sufficiently strong, confines the Hadley circulation to low latitudes. Real Hadley cells do not conserve angular momentum, and exhibit zonal winds increasing more slowly with latitude than expressed by Eq. (17); nevertheless, rotation generally confines Hadley circulations to low latitudes even in this case.

Over the past 30 years, many GCM studies have been performed to investigate how the Hadley circulation depends on planetary rotation rate and other parameters (Hunt 1979, Williams and Holloway 1982, Williams 1988a b; Del Genio and Suozzo 1987; Navarra and Boccaletti 2002. Walker and Schneider 2005, 2006; Kaspi and Showman 2012). Figure 12 illustrates an example from Kaspi and Showman (2012), showing GCM experiments for planets with no seasonal cycle forced by equator-pole heating gradients. Models were performed for rotation rates ranging from $1 / 16^{\text {th }}$ to 4 times that of Earth (top to bottom panels in Figure 12, respectively). Other parameters in these experiments, including solar flux, planetary radius and gravity, and atmospheric mass are all Earth-like. In general, the Hadley circulation consists of a cell in each hemisphere extending from the equator toward higher latitudes (dark red and blue regions in Figure 12). As the air in the upper branch moves poleward, it accelerates eastward by the Coriolis force, leading to the so-called subtropical jets whose amplitudes peak at the poleward edge of the Hadley cell (such subtropical jets are shown schematically in Figure 2). At sufficiently low rotation rates (top two panels of Figure 12, the Hadley circulation is nearly global, extending from equator to pole in both hemispheres, with the subtropical jets peaking at latitudes of $\sim 60^{\circ}$. Such models constitute "all tropics" worlds lacking any high-latitude extratropical baroclinic zone. At faster rotation rates (bottom five panels), the Hadley circulation - and the associated subtropical jets-become confined closer to the equator, and the higher latitudes develop an extratropical zone with baroclinic instabilities. The emergence of mid- and high-latitude eddy-driven jets in the extratropics, with eastward surface wind, can be seen in these rapidly rotating cases (cf Section 2.1.4. As shown in Figure 12, the tropospheric temperatures are relatively constant with latitude across the Hadley cell and begin decreasing poleward of the subtropical jets near its poleward edge. The resulting equator-to-pole temperature differences are small at slow rotation and increase with increasing rotation rate (Figure 12).

Seasonality exerts a strong effect on the Hadley circulation. When a planet's obliquity is non-zero, the substellar latitude oscillates between hemispheres, crossing the equator during equinox and reaching a peak excursion from the equator at solstice. The rising branch of the Hadley circulation tends to follow the latitude of maximum sunlight and therefore oscillates between hemispheres as well 11 Near equinox, the rising branch lies close to the equator, with Hadley cells of approximately equal strength in each hemisphere (cf, Figure 12). Near solstice, the rising branch lies in the summer hemisphere. As before, two cells exist, with air in one cell (the "winter" cell) flowing across the equator and descending in the winter hemisphere, and air in the other cell (the "summer" cell) flowing poleward toward the summer pole. Generally, for obliquities relevant to Earth, Mars, and Titan, the (cross-equatorial) winter cell is much stronger than the summer cell (see e.g., Peixoto and Oort 1992. pp. 158-160, for the Earth case). The zonal wind structure at solstice and equinox also differ significantly. The zonal wind in the ascending branch is generally weak, and, the greater its latitude, the lower its angular momentum per unit mass. If the ascending branch lies at latitude $\phi_{0}$ and exhibits zero zonal wind, and if the upper branch of the Hadley circulation conserves angular momentum, the zonal wind in the upper branch is

$$
u=\Omega a \frac{\cos ^{2} \phi_{0}-\cos ^{2} \phi}{\cos \phi} .
$$

Notice that this equation implies strong westward (negative) wind speeds near the equator if $\phi_{0}$ is displaced off the equator-a result of the fact that, in the winter cell, air is moving away from the rotation axis as it approaches the equator. Just such a phenomenon of strong westward equatorial wind under solsticial conditions can be seen in both idealized, axisymmetric models of the Hadley cell (e.g., Lindzen and Hou 1988, Caballero et al. 2008; Mitchell et al. 2009) and full GCM simulations performed under conditions of high obliquity (Williams and Pollard 2003). Nevertheless, eddies can exert a considerable effect on the Hadley cell, which will cause deviations from Eq. (18).

The Hadley circulation can exhibit a variety of possible behaviors depending on the extent to which the circula-

\footnotetext{
${ }^{11}$ When the atmospheric heat capacity is large, the response will generally be phase lagged with respect to the stellar heating pattern.
} 

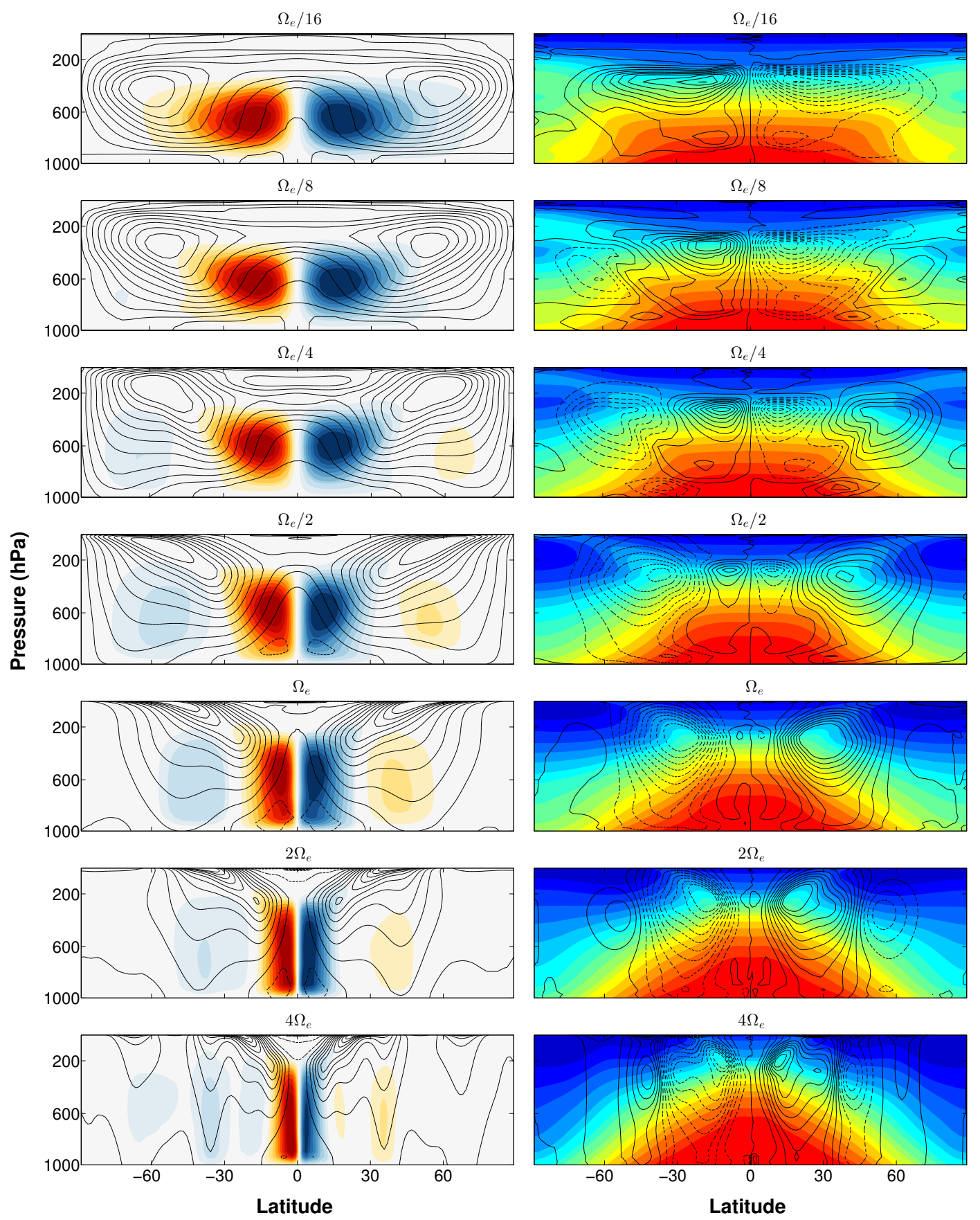

$\Omega_{e} / 8$

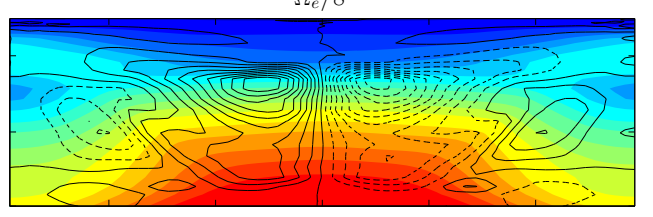

$\Omega_{e} / 4$

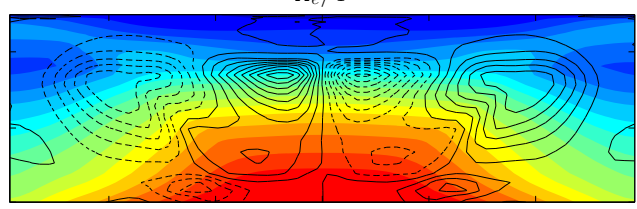

$\Omega_{e} / 2$

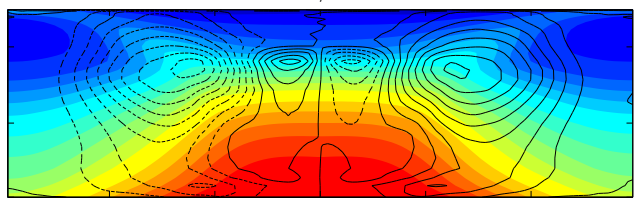

$\Omega_{e}$

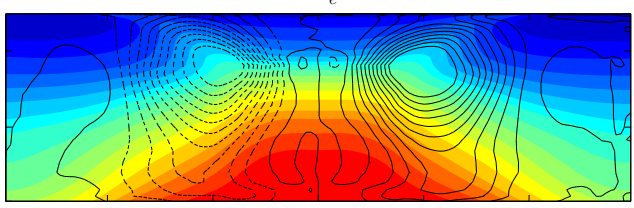

$2 \Omega_{e}$

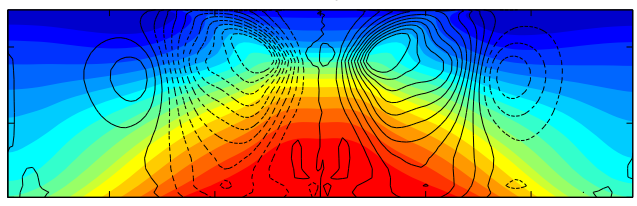

$4 \Omega_{e}$

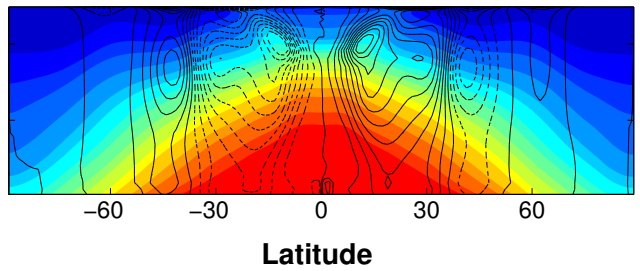

Fig. 12.- Zonal-mean circulation for a sequence of idealized GCM experiments of terrestrial planets from Kaspi and Showman (2012), showing the dependence of the Hadley circulation on planetary rotation rate. The models are driven by an imposed equator-pole insolation pattern with no seasonal cycle. The figure shows seven experiments with differing planetary rotation rates, ranging from $1 / 16^{\text {th }}$ to four times that of Earth from top to bottom, respectively. Left column: Thin black contours show zonal-mean zonal wind; the contour interval is $5 \mathrm{~m} \mathrm{~s}^{-1}$, and the zero-wind contour is shown in a thick black contour. Orange/blue colorscale depicts the mean-meridional streamfunction, with blue denoting clockwise circulation and orange denoting counterclockwise circulation. Right column: colorscale shows zonal-mean temperature. Contours show zonal-mean meridional eddy-momentum flux, $\overline{u^{\prime} v^{\prime}} \cos \phi$. Solid and dashed curves denote positive and negative values, respectively (implying northward and southward transport of eastward eddy momentum, respectively). At slow rotation rates, the Hadley cells are nearly global, the subtropical jets reside at high latitude, and the equatorpole temperature difference is small. The low-latitude meridional momentum flux is equatorward, leading to equatorial superrotation (eastward winds at the equator) in the upper troposphere. At faster rotation rates, the Hadley cells and subtropical jets contract toward the equator, an extratropical zone, with eddy-driven jets, develops at high latitudes, and the equator-pole temperature difference is large. The low-latitude meridional momentum flux is poleward, resulting from the absorption of equatorward-propagating Rossby waves coming from the extratropics. 
tion in the upper branch is angular-momentum conserving. The different regimes can be illuminated by considering the zonal-mean zonal wind equation, written here for the 3D primitive equations in pressure coordinates on the sphere (cf Held 2000, Section 6)

$\frac{\partial \bar{u}}{\partial t}=(f+\bar{\zeta}) \bar{v}-\bar{\omega} \frac{\partial \bar{u}}{\partial p}-\frac{1}{a \cos ^{2} \phi} \frac{\partial\left(\cos ^{2} \phi \overline{u^{\prime} v^{\prime}}\right)}{\partial \phi}-\frac{\partial\left(\overline{u^{\prime} \omega^{\prime}}\right)}{\partial p}$

where $\omega=d p / d t$ is the vertical velocity in pressure coordinates, $d / d t$ is the advective derivative, and as before the overbars and primes denote zonal averages and deviations therefrom. In a statistical steady state, the lefthand side of Equation (19) is zero. Denoting the eddy terms (i.e., the sum of the last two terms on the righthand side) by $-S$, we obtain

$$
(f+\bar{\zeta}) \bar{v}=\bar{\omega} \frac{\partial \bar{u}}{\partial p}+S
$$

For Earth, it turns out that the vertical advection by the mean flow (first term on righthand side) does not play a crucial role, at least for a discussion of the qualitative behavior, so that the zonal momentum balance for the upper branch of the Hadley circulation can be written (e.g., Held 2000, Schneider 2006, Walker and Schneider 2006)

$$
(f+\bar{\zeta}) \bar{v}=f\left(1-R o_{H}\right) \bar{v} \approx S
$$

where the Rossby number associated with the Hadley circulation is defined as $R o_{H}=-\bar{\zeta} / f$.

The Hadley circulation exhibits distinct behavior depending on whether the Rossby number, $R o_{H}$, is large or small. Essentially, $R o_{H}$ is a non-dimensional measure of the effect of eddies on the Hadley cell (e.g., Held 2000; Schneider 2006; Walker and Schneider 2006; Schneider and Bordoni 2008). The meridional width, amplitude, temperature structure, and seasonal cycle of the Hadley circulation depend on the relative roles of thermal forcing (in the form of meridional heating gradients) and mechanical forcing (in the form of $S$ ). The Hadley circulation will respond differently to a given change in thermal forcing depending on whether eddy forcing is negligible or important.

Theories currently exist for the $R_{H} \rightarrow 1$ and $\mathrm{Ro}_{H} \rightarrow 0$ limits, although not yet for the more complex intermediate case which seems to be representative of Earth and perhaps planets generally.

When eddy-induced accelerations are negligible, then $S=0$, and for non-zero circulations the absolute vorticity must then be zero within the upper branch, i.e., $f+\bar{\zeta}=0$, or, in other words, $R o_{H} \rightarrow 1$. The definitions of relative vorticity and angular momentum imply that $f+\bar{\zeta}=$ $\left(a^{2} \cos \phi\right)^{-1} \partial \bar{m} / \partial \phi$, from which it follows that a circulation with zero absolute vorticity exhibits angular momentum per mass that is constant with latitude. This is the angular-momentum conserving limit mentioned previously and, for atmospheres experiencing a heating maximum at the equator, would lead to a zonal-wind profile obeying (17) in the upper branch. Several authors have explored theories of such circulations, both for annual-mean conditions and including a seasonal cycle (e.g., Held and Hou 1980, Lindzen and Hou 1988; Fang and Tung 1996, Polvani and Sobel 2002; Caballero et al. 2008; Adam and Paldor 2009. 2010). Given the angular-momentum conserving wind in the upper branch (Equation 17), and assuming the near-surface wind is weak - a result of surface friction-the mean vertical shear of the zonal wind between the surface and upper troposphere is therefore known. The tropospheric meridional temperature gradient can then be obtained from the thermal-wind balance (2), or from generalizations of it that include curvature terms on the sphere (cf Held and Hou 1980). When the Hadley cell is confined to low latitudes, this leads to a quartic dependence of the temperature on latitude; for example, in the Held and Hou (1980) model, the potential temperature at a mid-tropospheric level is

$$
\theta=\theta_{\text {equator }}-\frac{\Omega^{2} \theta_{0}}{2 g a^{2} H} y^{4}
$$

where $H$ is the vertical thickness of the Hadley cell, $y$ is northward distance from the equator, and $\theta_{\text {equator }}$ is the potential temperature at the equator. This dependence implies that the temperature varies little with latitude across most of the Hadley cell but plummets rapidly near the poleward edges of the cell. In this $R_{H} \rightarrow 1$ regime, the strength of the Hadley cell follows from the thermodynamic energy equation, and in particular from the radiative heating gradients (with heating near the equator and cooling in the subtropics). The Hadley cell also has finite meridional extent that is determined by the latitude at which the integrated cooling away from the equator balances the integrated heating near the equator. The Hadley circulation in this limit is thermally driven.

On the other hand, eddy-momentum accelerations $S$ are often important and can play a defining role in shaping the Hadley cell properties. In the limit $R o_{H} \ll 1$, the strength of the Hadley circulation is determined not by the thermal forcing (at least directly) but rather by the eddymomentum flux divergences: because the absolute vorticity at $R o_{H} \ll 1$ is approximately $f$, the meridional velocity in the Hadley cell is given, via Equation 21, by $\bar{v} \approx S / f$, at least under conditions where the vertical momentum advection of the mean flow can be neglected. Generally, in the Earth and Mars-like context, the primary eddy effects on the Hadley cell result from the equatorward propagation of Rossby waves generated in the baroclinincally unstable zone in the midlatitudes (Section 2.1.4). The zonal phase velocities of these waves, while generally westward relative to the peak speeds of the eddy-driven jet, are eastward relative to the ground. These Rossby waves propagate into the subtropics (causing a poleward flux of eddy angular momentum visible in the bottom three panels of Figure 12, where they reach critical levels on the flanks of the subtropical jets (Randel and Held 1991). The resulting wave absorption generally causes a westward wave-induced acceleration of the zonal-mean zonal flow, which removes angular momentum from the poleward-flowing air in the up- 
per branch of the Hadley circulation. Therefore, the angular momentum decreases poleward with distance away from the equator in the upper branch of the Hadley cell. As a result, although the zonal wind still increases with latitude away from the equator, it does so more gradually than predicted by Equation (17); for example, at $20^{\circ}$ latitude, the zonal-mean zonal wind speed in Earth's upper troposphere is only $\sim 20 \mathrm{~m} \mathrm{~s}^{-1}$, significantly weaker than the $\sim 60 \mathrm{~m} \mathrm{~s}^{-1}$ predicted by Equation 17 . In turn, the weaker vertical shear of the zonal wind implies a weaker meridional temperature gradient through thermal wind (Equation 2), leading to a meridional temperature profile than can remain flatter over a wider range of latitudes than predicted by Equation 22) (see Farrell (1990) for examples of this phenomenon in the context of a simple, axisymmetric model).

Real planets probably lie between these two extremes. On Earth, observations and models indicate that eddy effects on the Hadley cell are particularly important during spring and fall equinox and in the summer hemisphere cell, whereas the winter hemisphere (cross-equatorial) Hadley cell is closer to the angular momentum conserving limit (e.g., Kim and Lee 2001, Walker and Schneider 2005, 2006, Schneider and Bordoni 2008; Bordoni and Schneider 2008, 2010). In particular, the Rossby numbers $R o_{H}$ vary from $\lesssim 0.3-0.4$ in the equinoctal and summer cells to $\gtrsim 0.7$ in the winter cell. Physically, the mechanisms for the transition between these regimes involve differences in an eddymean-flow feedback between equinoctal and solsticial conditions (Schneider and Bordoni 2008, Bordoni and Schneider 2008). During the equinox, the ascending branch of the Hadley cell lies near the equator, and the upper branch transports air poleward-i.e., toward the rotation axisinto both hemispheres. Angular momentum conservation therefore implies that the upper branch of the Hadley cell exhibits eastward zonal flow (Eq. 17), allowing the existence of critical layers and the resultant absorption of the equatorward-propagating Rossby waves from midlatitudes. Eddies therefore play a crucial role ( $S$ is large and $R o_{H}$ is small). During solstice, the ascending branch of the Hadley cell is displaced off the equator into the summer hemisphere. Air flows poleward in the upper branch of the summer cell, again leading to eastward zonal flow, the existence of critical layers, and significant eddy influences. On the other hand, air in the winter-hemisphere cell rises in the subtropics of the summer hemisphere and flows across the equator to the winter hemisphere, where it descends in the subtropics. Because this equatorward motion moves the air away from the rotation axis, angular momentum conservation leads to a broad region of westward zonal winds across much of the winter cell (Eq. 18, Lindzen and Hou 1988). Because the equatorward-propagating midlatitude eddies exhibit eastward zonal phase speeds (relative to the ground), the winter-hemisphere cell therefore lacks critical layers and is largely transparent to these waves. (Indeed, most of them have already reached critical levels and been absorbed before even reaching the latitude of the winter cell). The result is a much smaller net absorption of eddies, and therefore a winter hemisphere Hadley cell whose upper branch is much closer to the angular-momentum conserving limit.

Other feedbacks between Hadley cells and eddies are possible, particularly on tidally locked exoplanets where the day-night heating pattern dominates. In particular, tidally locked exoplanets exhibit a strong day-night heating pattern that induces standing, planetary-scale tropical waves, which can drive an eastward (superrotating) jet at the equator (Section 2.2.3. Such superrotation corresponds to a local maximum at the equator of angular momentum per mass about the planetary rotation axis; by definition, this means that the meridional circulation in a Hadley cell, if any, must cross contours of constant angular momentum. This can only occur in the presence of eddy accelerations that alter the angular momentum of the air in the upper branch as it flows meridionally. Therefore, Hadley cells in the presence of superrotation jets tend to be far from the angular-momentum conserving limit. The term $\bar{\omega} \partial \bar{u} / \partial p$ in Equation 20 may be crucial on such planets, unlike the typical case on Earth.

Shell and Held (2004) explored the interaction between superrotation and the Hadley circulation in a zonally symmetric, 1-1/2 layer shallow-water model where the effect of the tropical eddies was parameterized as a specified eastward acceleration at the equator. They found four classes of behavior depending on the strength of the eddy forcing. For sufficiently weak zonal eddy acceleration, the model's Hadley circulation approached the angular-momentum conserving limit. For eastward eddy accelerations exceeding a critical value, however, two stable equilibria emerged for a given eddy acceleration. In one equilibrium, the Hadley cell is strong, and the massive advection from below of air with minimal zonal wind inhibits the ability of the eddy forcing to generate a strongly superrotating jet at the equator. As a result, angular momentum varies only modestly with latitude near the equator, promoting the ability of the Hadley cell to remain strong. In the other equilibrium, the Hadley cell and the associated vertical momentum advection are weak, so the eddy acceleration is therefore able to produce a fast superrotating jet. The corresponding thermal profile is close to radiative equilibrium, maintaining the Hadley cell in its weak configuration. Shell and Held (2004) showed that when the eddy forcing exceeds a second critical threshold, only the latter solution exists, corresponding to a weak Hadley cell and a strongly superrotating jet. For eddy forcing exceeding a third critical threshold, the Hadley circulation collapses completely, replaced by an eddy-driven meridional circulation with sinking motion at the equator and ascending motion off the equator. It will be interesting to use more sophisticated models to determine the extent to which these various regimes apply to terrestrial exoplanets, and, if so, the conditions for transitions between them.

\subsubsection{Wave adjustment}

As discussed earlier in this section, the slowly rotating regime generally exhibits small horizontal temperature con- 
trasts, which results from the existence of dynamical mechanisms that efficiently regulate the thermal structure. The Hadley circulation, discussed previously, is one such mechanism, and is particularly important in the latitudinal direction at large scales. Another key mechanism is adjustment of the thermal structure by gravity waves.

How does such wave adjustment work? When moist convection, radiative heating gradients, or other processes generate horizontal temperature variations, the resulting pressure gradients lead to the radiation of gravity waves. The horizontal convergence/divergence induced by these waves adjusts air columns up or down in such a way as to flatten the isentropes and therefore erase the horizontal temperature constrasts. This process, which is essentially the non-rotating endpoint of geostrophic adjustment, plays a key role in minimizing the horizontal temperature contrasts in the tropics; the resulting dynamical regime is often called the "weak temperature gradient" or WTG regime (Sobel et al. 2001; Sobel 2002).

This adjustment process is most simply visualized in the context of a one-layer fluid. Imagine a shallow, nonrotating layer of water in one spatial dimension, whose initial surface elevation is a step function. This step causes a sharp horizontal pressure gradient force, and this will lead to radiation of gravity waves (manifested here as surface water waves) in either direction. Figure 13 shows an analytical solution of this process from Kuo and Polvani (1997). The horizontal convergence/divergence induced by the waves changes the fluid thickness. Assuming the waves can radiate to infinity (or break at some distant location), the final state is a flat layer. When the fractional height variation is small, this adjustment process causes only a small horizontal displacement of fluid parcels; the adjustment is done by waves, not long-distance horizontal advection.

This wave-adjustment mechanism also acts to regulate the thermal structure in three-dimensional, continuously stratified atmospheres (e.g., Bretherton and Smolarkiewicz 1989, Sobel 2002). In a stratified atmosphere, horizontal temperature differences are associated with topographic variations of isentropes, which play a role directly analogous to the topography of the water surface in the example described above. Gravity waves induce horizontal convergence/divergence, which changes the vertical thickness of the fluid columns, thereby pushing the isentropes up or down. Assuming that planetary rotation is sufficiently weak, and that the waves can radiate to infinity, the final state is one with flat isentropes. Note that, if the initial fractional temperature contrast is small, this adjustment causes only a small lateral motion of fluid parcels.

Figure 14 demonstrates this process explicitly in a threedimensional, continously stratified atmosphere. There we show a solution of the global, three-dimensional primitive equations for an initial-value problem in which half of the planet (the "dayside") is initialized to have a potential temperature that is $20 \mathrm{~K}$ hotter than the other half (the "nightside"). The heating/cooling is zero so that the flow is adiabatic and isentropes are material surfaces. The top row shows the initial condition, and subsequent rows show the state at later times during the evolution. At early times $\left(0.5 \times 10^{4} \mathrm{~s}\right.$, second row $)$, waves begin to radiate away from the day-night boundary; they propagate across most of the planet by $\sim 2 \times 10^{4}$ s (third row). They also propagate upward where they are damped in the upper atmosphere, leaving the long-term state well-adjusted (fourth row). Note that, in the final state, the isentropes are approximately flat and the day-night temperature differences are greatly reduced over their initial values.

The timescales for this wave-adjustment process can differ significantly from the relevant advection and mixing timescales. Because the wave-adjustment mechanism requires the propagation of gravity waves, the timescale to adjust the temperatures over some distance $L$ is essentially the gravity wave propagation time over distance $L$, i.e., $\tau_{\text {adjust }} \sim L / N H$, where $N$ is the Brunt-Vaisala frequency and $H$ is a scale height. For example, Bretherton and Smolarkiewicz (1989) show how gravity waves adjust the thermal structure in the environment surrounding tropical cumulus convection; they demonstrate that the process operates on a timescale much shorter than the lateral mixing timescale. Moreover, if the wave propagation speeds differ significantly from the wind speeds, then $\tau_{\text {adjust }}$ can differ significantly from the horizontal advection time, $\tau_{\mathrm{adv}} \sim L / U$. In the example shown in Fig. 14 for example, the wave speeds are $c \sim N H \sim 200 \mathrm{~m} \mathrm{~s}^{-1}$ (using $N \approx 0.02 \mathrm{~s}^{-1}$ and $H \approx 10 \mathrm{~km}$ ), but the peak tropospheric wind speeds in this simulation are almost ten times smaller. This implies that the timescale for wave propagation is nearly ten times shorter than the timescale for air to advect over a given distance.

Understanding the conditions under which the WTG regime breaks down is critical for understanding the atmospheric stability, climate, and habitability of exoplanets. Synchronously rotating planets exhibit permanent day and night sides, and for such atmospheres to remain stable against collapse, they must remain sufficiently warm on the nightside (Joshi et al. 1997). Crudely, one might expect that dynamics fails to erase the day-night temperature contrast when the radiative timescale becomes shorter than the relevant dynamical timescale. Most previous exoplanet literature has assumed that the relevant comparison is between the radiative and advective timescales (e.g., Showman et al. 2010, Cowan and Agol 2011). However, when the wave-adjustment time is short, a comparison between the radiative and wave-adjustment timescales may be more appropriate. For typical terrestrial-planet parameters $\left(N \approx 10^{-2} \mathrm{~s}^{-1}, H=10 \mathrm{~km}\right.$ and taking $L$ to be a typical terrestrial-planet radius of $6000 \mathrm{~km}$ ) yields $\tau_{\text {adjust }} \sim$ $10^{5} \mathrm{sec}$. This would suggest that on planets with radiative time constants $\lesssim 10^{5} \mathrm{sec}$, the waves are damped, the WTG regime breaks down, and large day-night temperature differences may occur. Earth, Venus, and Titan are safely out of danger, but Mars is transitional, and any exoplanet whose atmosphere is particularly thin and/or hot is also at risk. Nevertheless, subtleties exist. For example, there exists a 


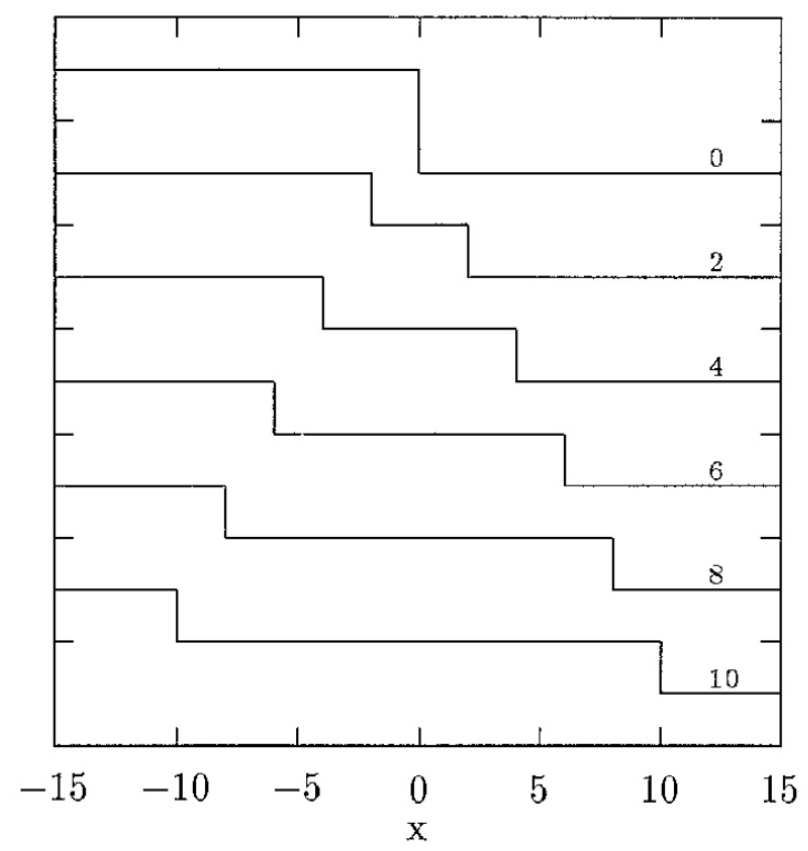

Fig. 13. - An analytic solution of the wave adjustment process in a one-dimensional, nonrotating shallow-water fluid. Each curve shows the elevation of the water surface (with an arbitrary vertical offset for clarity) versus horizontal distance at a particular time. Numbers labeling each curve give time. The initial condition is shown at the top, and subsequent states are shown underneath. Note how all the topography is "carried away" by the waves. From Kuo and Polvani (1997).

wide range of wave-adjustment timescales associated with waves of differing wavelengths and phase speeds; moreover, the timescale for waves to propagate vertically out of the troposphere is not generally equivalent to the timescale for them to propagate horizontally across a hemisphere. Although the wave-adjustment process is fundamentally a linear one, nonlinearities may become important at high amplitude as the WTG regime breaks down and the fractional day-night temperature difference becomes large. The horizontal advection time may play a key role in this case. Further work is warranted on the precise conditions for WTG breakdown and the extent to which they can be packaged as timescale comparisons.

For simplicity, we have so far framed the discussion around non-rotating planets; to what extent does the mechanism carry over to rotating planets? Because horizontal Coriolis forces are zero at the equator, the picture has broad relevance for tropical meteorology even on rapidly rotating planets. More specifically, planetary rotation tends to trap tropical wave modes into an equatorial waveguide, whose meridional width is approximately the equatorial Rossby deformation radius, $L_{\mathrm{eq}}=(c / \beta)^{1 / 2}$, where $c$ is a typical gravity wave speed and $\beta=d f / d y$ is the derivative of the Coriolis parameter with northward distance; this tends to yield a characteristic waveguide width of order $(N H / \beta)^{1 / 2}$ in a continuously stratified atmosphere (see Matsuno (1966), Holton (2004, pp. 394-400, 429-432), or Andrews et al.(1987. pp. 200-208) for a discussion of equatorial wave trapping). Typical values are $L_{\mathrm{eq}} \sim 10^{3} \mathrm{~km}$ for
Earth and Mars. These equatorially trapped modes, including the Kelvin wave, Rossby waves, and mixed Rossbygravity waves - as well as smaller-scale gravity waves triggered by convection and other processes-can adjust the thermal state in a manner analogous to that described here. Because large-scale, equatorially trapped waves can propagate in longitude and height but not latitude, the adjustment process will occur more efficiently in the zonal than the meridional direction, and it will tend to be confined to within an equatorial deformation radius of the equator. Indeed, the mechanism described here helps to explain why the Earth's tropospheric tropical temperatures are nearly zonally uniform, and moreover shows how moist convection can regulate the thermal structure over wide areas of the tropics despite its sporadic occurrence (Bretherton and Smolarkiewicz 1989). On slowly rotating planetsincluding Venus, Titan, and tidally locked super Earths, where the deformation radius is comparable to or greater than the planetary radius - this wave-adjustment processes will not be confined to low latitudes but will act globally to mute horizontal temperature contrasts. In large measure, this mechanism is responsible for the small horizontal temperature contrasts observed in the tropospheres of Venus and Titan.

Note that the WTG regime (when it occurs) applies best in the free troposphere, where friction is weak and waves are free to propagate; in the frictional boundary layer near a planet's surface, the scaling will not generally hold and there may exist substantial horizontal temperature gradi- 

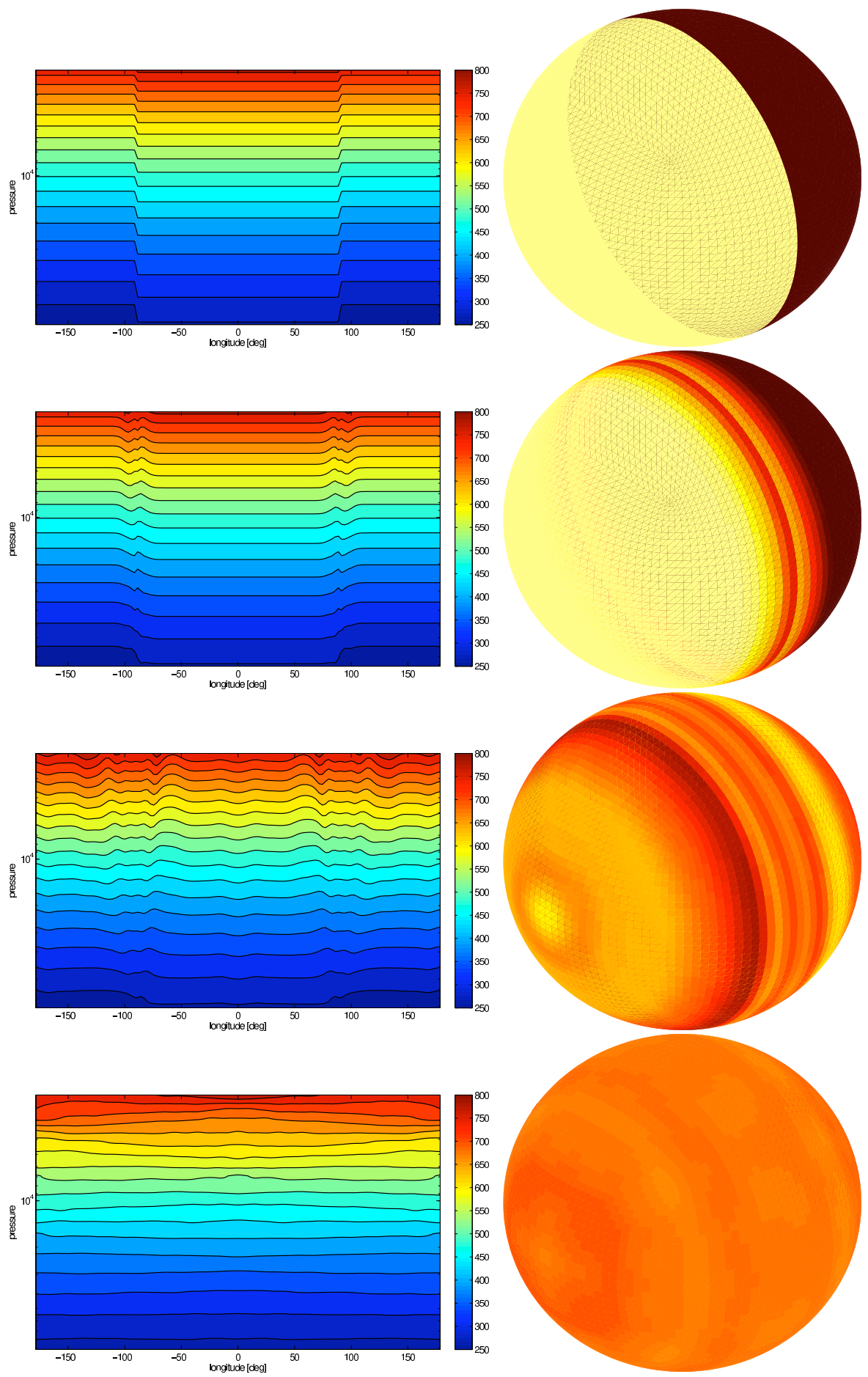

Fig. 14. - Numerical solution of wave adjustment on a spherical, non-rotating terrestrial planet with the radius and gravity of Earth. We solved the global, three-dimensional primitive equations, in pressure coordinates, using the MITgcm. Half of the planet (the "nightside") was initialized with a constant (isothermal) temperature of $T_{\text {night }}=250 \mathrm{~K}$, corresponding to a potential temperature profile $\theta_{\text {night }}=T_{\text {night }}\left(p_{0} / p\right)^{\kappa}$, where $\kappa=R / c_{p}=2 / 7$ and $p_{0}=1$ bar is a reference pressure. The other half of the planet (the "dayside") was initialized with a potential temperature profile $\theta_{\text {night }}(p)+\Delta \theta$, where $\Delta \theta=20 \mathrm{~K}$ is a constant. Domain extends from approximately 1 bar at the bottom to $0.001 \mathrm{bar}$ at the top; equations were solved on a cubed-sphere grid with horizontal resolution of $\mathrm{C} 32(32 \times 32$ cells per cube face, corresponding to an approximate resolution of $2.8^{\circ}$ ) and 40 levels in the vertical, evenly spaced in log- $p$. The model includes a sponge at pressures less than 0.01 bar to absorb upward-propagating waves. This is an initial value problem; there is no radiative heating/cooling so that the flow is adiabatic. Left: Potential temperature (colorscale and contours) at the equator versus longitude and pressure; Right: Temperature at a pressure of 0.2 bar over the globe at times of 0 (showing the initial condition), $0.5 \times 10^{4} \mathrm{~s}, 3 \times 10^{4} \mathrm{~s}$, and the final long-term state once the waves have propagated into the upper atmosphere. Air parcels move by only a small fraction of a planetary radius during the adjustment process, but the final state nevertheless corresponds to nearly flat isentropes with small horizontal temperature variations on isobars. 
ents. For example, Joshi et al. (1997) and Merlis and Schneider (2010) present terrestrial exoplanet simulations exhibiting weak temperature gradients in the free troposphere (day-night contrasts $\lesssim 3 \mathrm{~K}$ ) but larger day-night temperature contrasts at the surface (reaching $\sim 30-50 \mathrm{~K}$ ).

\subsubsection{Equatorial superrotation}

Recent theoretical work suggests that many tidally locked terrestrial exoplanets will exhibit a fast eastward, or superrotating, jet stream at the equator. More specifically, superrotation is defined as atmospheric flow whose angular momentum (per unit mass) about the planet's rotation axis exceeds that of the planetary surface at the equator ${ }^{12}$ In our solar system, the tropical atmospheres of Venus, Titan, Jupiter, and Saturn all superrotate. Even localized layers within Earth's equatorial stratosphere exhibit superrotation, part of the so-called "Quasi-Biennial Oscillation" (Andrews et al. 1987). In contrast, Uranus and Neptune, as well as the tropospheres of Earth and Mars, exhibit mean westward equatorial flow (subrotation). Interestingly, threedimensional circulation models of synchronously rotating exoplanets, which are subject to a steady, day-night heating pattern, have consistently showed the emergence of such superrotation - both for terrestrial planets (Joshi et al. 1997; Merlis and Schneider 2010, Heng and Vogt 2010, Edson et al.2011; Wordsworth et al. 2011) and hot Jupiters (Showman and Guillot 2002; Cooper and Showman 2005; Showman et al. 2008, 2009. 2013; Menou and Rauscher 2009: Rauscher and Menou 2010, Heng et al. 2011b a; Perna et al. 2010, 2012). Figure 15 shows examples from several recent studies.

Equatorial superrotation is interesting for several reasons. First, it influences the atmospheric thermal structure and thus plays an important role in shaping observables. When radiative and advective timescales are similar, the superrotation can cause an eastward displacement of the thermal field that influences infrared light curves and spectra (e.g., Showman and Guillot 2002). An eastward displacement of the hottest regions from the substellar point has been observed in light curves of the hot Jupiter HD 189733b (Knutson et al.2007) and it may also be detectable for synchronously rotating super Earths with next-generation observatories (Selsis et al.|2011). The thermal structure of the leading and trailing terminators may also differ. Second, superrotation is dynamically interesting; understanding the mechanisms that drive superrotation in the exoplanet context may inform our understanding of superrotation within the solar system (and vice versa). The equator is the region farthest from the planet's rotation axis; therefore, a superrotating jet corresponds to a local maximum of angular momentum per mass about the planet's rotation axis. Maintaining a superrotating equatorial jet against friction or other processes therefore requires angular momentum to be transported up-gradient from regions where it is low (outside the

\footnotetext{
${ }^{12}$ According to this definition, most eastward jets at mid- to high latitudes are not superrotating.
}

jet) to regions where it is high (inside the jet). Hide (1969) showed that the necessary up-gradient angular-momentum transport must be accomplished by waves or eddies.

In models of synchronously rotating exoplanets, the defining feature that allows emergence of strong superrotation is the steady day-night heating contrast. Models that include strong dayside heating and nightside cooling-fixed in longitude due to the synchronous rotation-generally exhibit a broad, fast eastward jet centered at the equator. In contrast, otherwise similar models with axisymmetric forcing (i.e., an equator-to-pole heating gradient with no diurnal cycle) exhibit only weak eastward or westward winds at the equator, often accompanied by fast eastward jets in the mid-to-high latitudes (Figure 15).

Although the Earth's troposphere is not superrotating, it does exhibit tropical zonal heating anomalies due to longitudinal variations in the surface type (land versus ocean), sea-surface temperature, and prevalence of cumulus convection near the equator (Schumacher et al. 2004, Kraucunas and Hartmann 2005; Norton 2006). Qualitatively, these tropical heating/cooling anomalies resemble the daynight heating contrast on a synchronously rotating exoplanet, albeit at higher zonal wavenumber and lower amplitude. Starting in the 1990s, several authors in the terrestrial literature demonstrated using GCMs that, if sufficiently strong, these types of tropical heating anomalies can drive equatorial superrotation (Suarez and Duffy 1992; Saravanan 1993; Hoskins et al. 1999: Kraucunas and Hartmann 2005, Norton 2006). The qualitative similarity in the forcing and the response suggests that the mechanism for superrotation is the same in both the terrestrial and the exoplanet models.

What is the mechanism for the equatorial superrotation occurring in these models? Motivated by arguments analogous to those in Section 2.1.4 Held (1999b) and Hoskins et al. (1999) suggested heuristically that the superrotation results from the poleward propagation of Rossby waves generated at low latitudes by the tropical zonal heating anomalies; subsequent authors have likewise invoked this conceptual framework in qualitative discussions of the topic (e.g. Tziperman and Farrell|2009, Edson et al. 2011;:Arnold et al. 2012). At its essence, the hypothesis is attractive, since it is simple, based on the natural relationship of the meridional propagation directions of Rossby waves to the resulting eddy velocity phase tilts, and seemingly links extratropical and tropical dynamics. Nevertheless, the idea remains qualitative, and its relevance to the type of equatorial superrotation seen in exoplanet GCMs has not been demonstrated.

Moreover, challenges exist. First, unlike in the extratropics, large-scale baroclinic equatorial wave modes in the tropics are equatorially trapped, confined to a wave guide whose meridional width is approximately the equatorial Rossby deformation radius. Such waves-including the Kelvin waves, equatorial Rossby waves, and the mixed Rossby-gravity wave-can propagate in longitude and height but not latitude. Unlike the case of the barotropic 


\section{Synchronously locked}
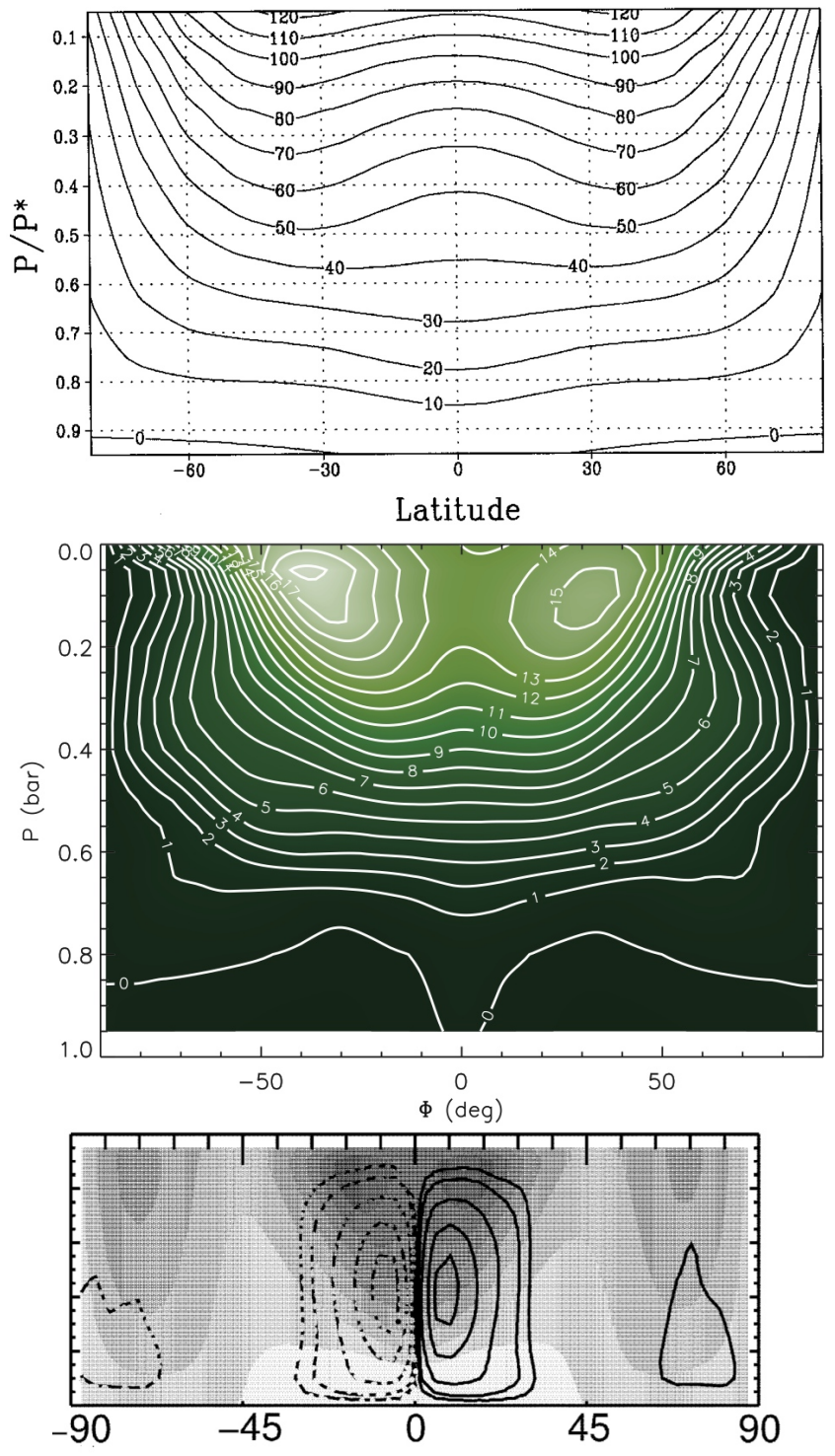
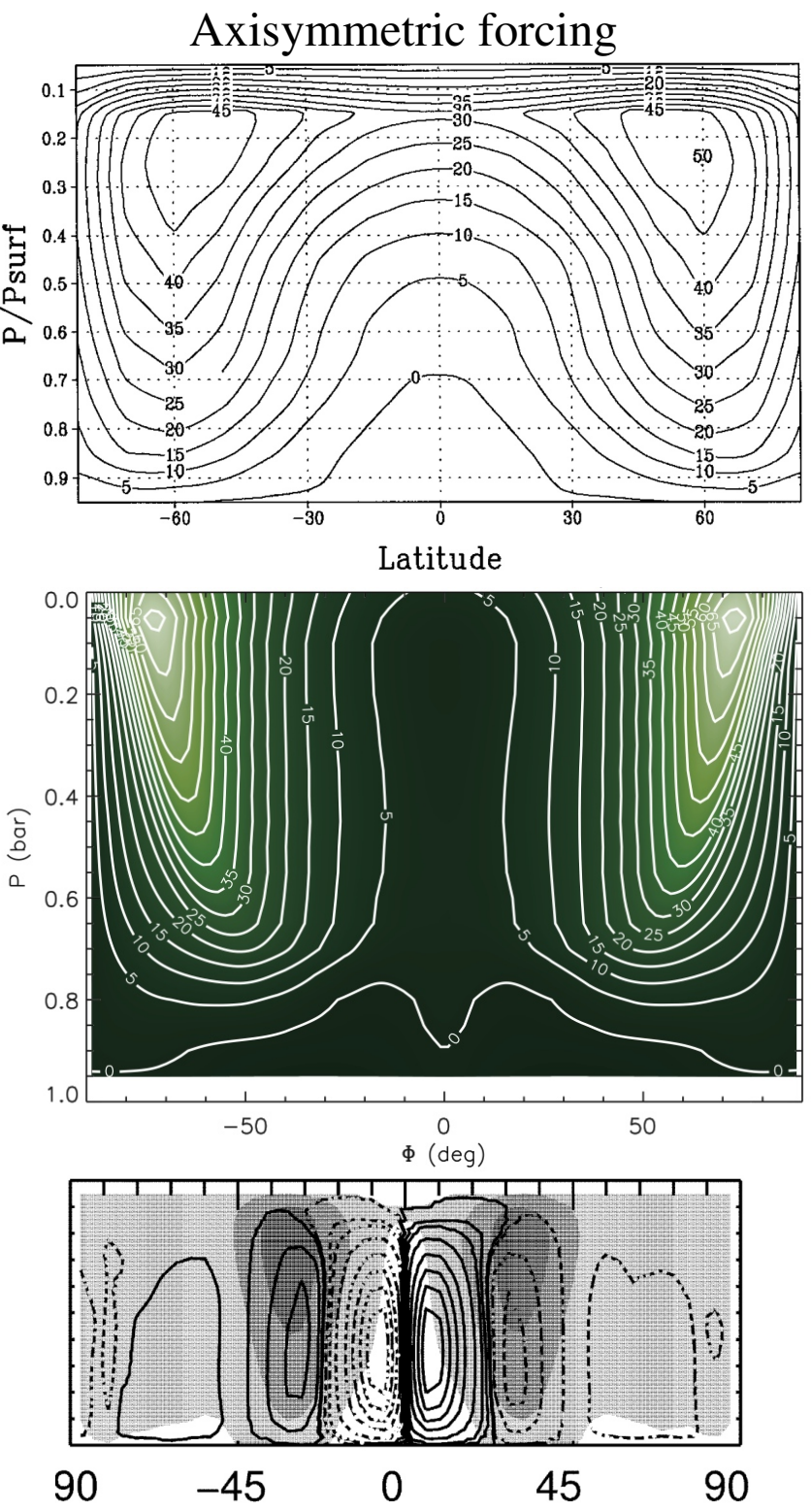

Fig. 15.-Zonal-mean zonal winds (contours) versus latitude (abscissa) and pressure (ordinate) from recent studies illustrating the development of equatorial superrotation in models of synchronously rotating terrestrial exoplanets. In each case, the left column shows a synchronously rotating model with a steady, day-night heating pattern, and the right model shows an otherwise similar control experiment with axisymmetric heating (i.e., no day-night pattern). Top: From Joshi et al. (1997); rotation period is 16 Earth days. Contours give zonal-mean zonal wind in $\mathrm{m} \mathrm{s}^{-1}$. Middle: Heng and Vogt (2010); rotation period is 37 days (left) and 20 days (right). Contours give zonal-mean zonal wind in $\mathrm{m} \mathrm{s}^{-1}$. Bottom: Edson et al. (2011); rotation period is 1 Earth day. Greyscale gives zonal-mean zonal wind; eastward is shaded, with peak values (in dark shades) reaching $\sim 30 \mathrm{~m} \mathrm{~s}^{-1}$. Contours give mean-meridional streamfunction. In all the models, the synchronously rotating variants exhibit a strong, broad eastward jet centered at the equator, whereas the axisymmetrically forced variants exhibit weaker eastward or westward flow at the equator, with eastward jets peaking in the mid-to-high latitudes. 
Rossby waves discussed in Section 2.1.3, simple analytic solutions for such waves (see, e.g., Matsuno 1966; Holton 2004; Andrews et al. 1987) exhibit no meridional momentum flux. Second, in the context of slowly rotating exoplanets, these waves exhibit meridional scales typically extending from the equator to the pole (e.g., Mitchell and Vallis 2010), leaving little room for meridional propagation. Given these issues, it is unclear that the paradigm of waves propagating from one latitude to another applies.

It can be shown explicitly that the barotropic theory for the interaction of meridionally propagating Rossby waves with the mean flow-which explains the emergence of eddy-driven jets in the extratropics (Section 2.1.4) - fails to explain the equatorial superrotation emerging from exoplanet GCMs (Showman and Polvani 2011). Many GCMs of synchronously rotating exoplanets exhibit circulation patterns - including equatorial superrotation - that, to zeroth order, are mirror symmetric about the equator (e.g., Showman and Guillot 2002, Cooper and Showman 2005, Showman et al. 2008, 2009, 2013, Heng et al. 2011b a; Showman and Polvani 2010, 2011; Rauscher and Menou 2010, 2012, Perna et al. 2010, 2012). For a flow with such symmetry, the relative vorticity is antisymmetric about, and zero at, the equator - at all longitudes, not just in the zonal mean. Under these conditions, the forcing $\overline{\zeta^{\prime} F^{\prime}}$ equals zero in Equations (13) and (14). Equation (14) therefore predicts that the zonal-mean zonal wind is zero at the equatorinconsistent with the equatorial superrotation emerging in the GCMs.

Showman and Polvani (2011) offered an alternate theory to overcome these obstacles and show how the equatorial superrotation can emerge from the day-night thermal forcing on synchronously rotating exoplanets. They presented an analytic theory using the 1-1/2 layer shallow-water equations, representing the flow in the upper troposphere; they demonstrated the theory with a range of linear and nonlinear shallow-water calculations and full, 3D GCM experiments. In their theory, the day-night forcing generates standing, planetary-scale Rossby and Kelvin waves, analogous to those described in analytic solutions by Matsuno (1966) and Gill (1980). See Figure 2 for a schematic illustration of these waves in the context of the overall circulation of a synchronously rotating exoplanet. In Showman and Polvani (2011)'s model, these baroclinic wave modes are equatorially trapped and, in contrast to the theory described in Section 2.1.4. exhibit no meridional propagation. The Kelvin waves, which straddle the equator, exhibit eastward (group) propagation, while the Rossby modes, which lie on the poleward flanks of the Kelvin wave, exhibit westward (group) propagation. Relative to radiativeequilibrium solutions, this latitudinally varying zonal propagation causes an eastward displacement of the thermal and pressure fields at the equator, and a westward displacement of them at higher latitudes (see Figure 16 for an analytic solution demonstrating this behavior). In turn, these displacements naturally generate an eddy velocity pattern with velocities tilting northwest-southeast in the northern hemisphere and southwest-northeast in the southern hemisphere, which induces an equatorward flux of eddy momentum (Figure 16) and equatorial superrotation.

It is crucial to note that, in the Showman and Polvani (2011) theory, the superrotation results not from wave tilts associated with meridional wave propagation (which cannot occur for these equatorially trapped modes), but rather from the differential zonal propagation of these Kelvin and Rossby modes. Indeed, in full, three-dimensional GCMs exhibiting equatorial superrotation in response to tropical heating anomalies, the tropical eddy response bears striking resemblance to these analytical, "Gill-type" solutions (Kraucunas and Hartmann 2005; Norton 2006, Caballero and Huber 2010, Showman and Polvani 2011; Arnold et al. 2012), providing further evidence that superrotation in these 3D models results from this mechanism. Interestingly, though, Showman and Polvani (2011) and Showman et al. (2013) showed that, even when thermal damping is sufficiently strong to inhibit significant zonal propagation of these wave modes, the multi-way force balance between pressure-gradient forces, Coriolis forces, advection, and (if present) frictional drag can in some cases lead to progradeequatorward and retrograde-poleward tilts in the eddy velocities, allowing an equatorward momentum convergence and equatorial superrotation. At face value, this latter mechanism does not seem to require appeal to wave propagation at all. See Showman and Polvani (2011) and Showman et al. (2013) for further discussion.

Nevertheless, real atmospheres contain a wide variety of wave modes, and there remains a need to better test and integrate these various mechanisms. Linear studies show that tropical convection on Earth generates two broad classes of wave mode-baroclinic, equatorially trapped waves that propagate in longitude and height but remained confined near the equator, and barotropic Rossby waves that propagate in longitude and latitude but exhibit no vertical propagation (e.g., Hoskins and Karoly 1981; Salby and Garcia 1987, Garcia and Salby 1987). By focusing on the 1-1/2 layer shallow-water model, Showman and Polvani (2011)'s study emphasized the equatorially trapped component and did not include a barotropic (meridionally propagating) mode. It would therefore be worth revisiting this issue with a multi-layer model that includes both classes of wave mode; such a model would allow a better test of the Held (1999b) hypothesis and allow a determination of the relative importance of the two mechanisms under various scenarios for day-night heating on exoplanets.

Several wave-mean-flow feedbacks exist that may influence the strength and properties of equatorial superrotation on exoplanets. First are the possible feedbacks with the Hadley circulation (Shell and Held 2004) described in Section 2.2.1. These feedbacks have yet to be explored in an exoplanet context.

Second, on planets rotating sufficiently rapidly to exhibit baroclinincally active extratopical zones, there is a possible feedback involving the effect of midlatitude eddies on the equatorial flow. In the typical Earth-like (non- 

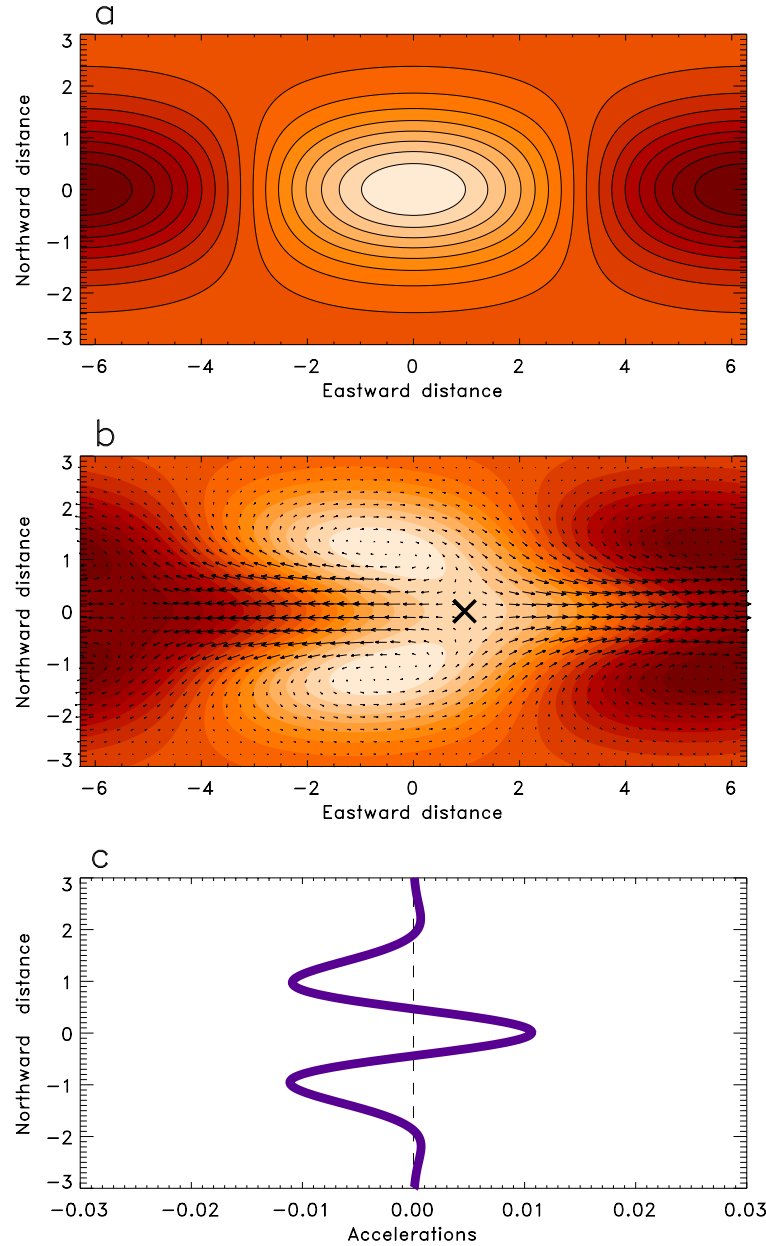

Fig. 16.- Analytical solutions of the shallow-water equations from Showman and Polvani (2011) showing how daynight thermal forcing on a synchronously rotating exoplanet can induce equatorial superrotation. (a) Spatial structure of the radiative-equilibrium height field versus longitude (abscissa) and latitude (ordinate). (b) Height field (colors) and eddy velocities (arrows) for steady, analytic solutions forced by radiative relaxation and linear drag, performed on the equatorial $\beta$ plane and analogous to the solutions of Matsuno (1966) and Gill (1980). The equatorial behavior is dominated by a standing, equatorially trapped Kelvin wave and the mid-to-high latitude behavior is dominated by an equatorially trapped Rossby wave. The superposition of these two wave modes leads to eddy velocities tilting northwest-southeast (southwest-northeast) in the northern (southern) hemisphere, as necessary to converge eddy momentum onto the equator and generate equatorial superrotation. (c) The zonal accelerations of the zonal-mean wind implied by the linear solution. The acceleration is eastward at the equator, implying that equatorial superrotation will emerge. Note that the equatorward momentum flux that induces superrotation results from differential zonal, rather than meridional, propagation of the wave modes; indeed, these modes are equatorially trapped and exhibit no meridional propagation at all. 
superrotating) regime, equatorward-propagating Rossby waves generated by midlatitude baroclinic instabilities can be absorbed on the equatorward flanks of the subtropical jets (Section 2.2.1), causing a westward acceleration that helps inhibit a transition to superrotation-even in the presence of tropical forcing like that shown in Figure 16. On the other hand, if such tropical forcing becomes strong enough to overcome this westward torque, a transition to superrotation nevertheless becomes possible. Once this occurs, the equatorward-propagating Rossby waves no longer encounter critical levels in the tropics, and the tropics become transparent to these waves, eliminating any westward acceleration associated with their absorption. This feedback suggests that, near the transition, modest increases in the tropical wave source can cause sudden and massive changes in the equatorial jet speed. Two-level Earth-like GCMs with imposed tropical eddy forcing confirm this general picture and show that, near the transition, hysteresis can occur (Suarez and Duffy 1992, Saravanan 1993, Held 1999b). Nevertheless, otherwise similar GCM experiments with a more continuous vertical structure suggest that the feedback in practice is not strong (Kraucunas and Hartmann 2005; Arnold et al. 2012), hinting that the 2-level models may not properly capture all the relevant dynamics. Additional work is needed to determine the efficacy of this feedback in the exoplanet context.

Third, Arnold et al. (2012) pointed out a feedback between the tropical waves (like those in Figure 16) and the superrotation. In the presence of stationary, zonally asymmetric tropical heating/cooling anomalies, the linear response comprises steady, planetary-scale Rossby waves (cf Figure 16, whose thermal extrema are phase shifted to the west of the substellar longitude due to the westward (group) propagation of the waves. In the presence of modest superrotation, the phase shifts are smaller, and the wave amplitudes are larger. A resonance occurs when the (eastward) speed of the superrotation equals the (westward) propagation speed of the Rossby waves, leading to very large Rossby wave amplitude for a given magnitude of thermal forcing. Arnold et al. (2012) show that this feedback leads to an increased convergence of eddy momentum onto the equator as the superrotation develops, accelerating the superrotation still further. This positive feedback drives the atmosphere toward the resonance; beyond the resonance, the feedback becomes negative. Arnold et al. (2012) demonstrated these dynamics in linear shallow-water calculations and full GCM experiments. Nevertheless, a puzzling aspect of the interpretation is that, for the equatorially trapped wave modes considered by Showman and Polvani (2011) and Arnold et al. (2012), the ability of the waves to cause equatorward momentum convergence depends not just on the Rossby waves but on both equatorially trapped Rossby and Kelvin waves, whose superposition-given the appropriate zonal phase offset of the two modes-leads to the necessary phase tilts and equatorward momentum fluxes to cause superrotation. The resonance described above would not apply to the Kelvin wave component, since its (group) propagation is eastward. Additional theoretical work may clarify the issue.

Edson et al. (2011) performed GCM simulations of synchronously rotating but otherwise Earth-like exoplanets over a broad range of rotation rates, and they found the existence of a sharp transition in the speed of superrotation as a function of rotation rate. The system exhibited hysteresis, meaning the transition from weak to strong superrotation occurred at a different rotation rate than the transition from strong to weak superrotation. The specific mechanisms remain unclear; nevertheless, the model behavior suggests that positive feedbacks, possibly analogous to those described above, help to control the superrotation in their models. The speed of superrotation likewise differed at differing rotation rates in the GCM simulations of Merlis and Schneider (2010), but the transition was a more gradual function of rotation rate, and these authors did not suggest the existence of any hysteresis.

Finally, we note that slowly rotating planets exhibit a natural tendency to develop equatorial superrotation even in the absence of day-night or other imposed eddy forcing. This has been demonstrated in a variety of GCM studies where the imposed thermal forcing does not include a diurnal cycle (i.e., where the radiative equilibrium temperature depends on latitude and pressure but not longitude) (e.g., Del Genio et al. 1993; Del Genio and Zhou 1996; Yamamoto and Takahashi 2003, Herrnstein and Dowling 2007; Richardson et al. 2007; Lee et al. 2007, Hollingsworth et al. 2007; Mitchell and Vallis 2010; Parish et al. 2011; Lebonnois et al. 2012). Superrotation of this type can be seen in the axisymmetrically forced, slowly rotating models from Kaspi and Showman (2012) in Figure 12 (top four panels) and Joshi et al. (1997) and Heng and Vogt (2010) in Figure 15. Although the zonal wind at the equator corresponds to a local minimum with respect to latitude, it is eastward, and the equatorial upper troposphere still comprises a local maximum of angular momentum per mass. Thus, even this configuration (unlike a case where the zonal-mean zonal wind at the equator is zero or westward) must be maintained by eddy transport of angular momentum to the equator. Just such eddy-momentum fluxes can be seen in Figure 12 in the slowly rotating cases exhibiting superrotation, eddy momentum converges onto the equator (opposite to the sign of the low-latitude eddymomentum fluxes in the faster-rotating cases lacking superrotation). Although the details are subtle, several authors have suggested that the superrotation in this class of model results from equatorward angular momentum transport by a barotropic instability of the subtropical jets (e.g. Del Genio et al. 1993; Del Genio and Zhou 1996; Mitchell and Vallis 2010). This mechanism is relevant to the superrotation on Venus and Titan (although the thermal tides, which represent a day-night forcing that can trigger global-scale wave modes, are relvant to those planets as well). See the chapter by Lebonnois et al. for more details. 


\subsection{Effect of day-night forcing}

Many observationally accessible exoplanets will be sufficiently close to their stars to be tidally despun to a synchronous rotation state. For such exoplanets, the slowly rotating, tropical dynamical regime will often go hand-inhand with a strong day-night thermal forcing arising from the fact that they have permanent daysides and nightsides. A major question is how the atmospheric circulation depends on the strength of this day-night forcing. Will the circulation exhibit an essentially day-night flow pattern? Or, instead, will the circulation respond primarily to the zonal-mean radiative heating/cooling, developing a zonally banded flow in response to the planetary rotation? What is the amplitude of the day-night temperature difference? Is there a danger of the atmosphere freezing out on the night side?

To pedagogically illustrate the dynamics involved, we present in Figure 17 four numerical solutions of the shallow-water equations, representing the upper tropospheric flow on a sychronously rotating, terrestrial exoplanet. The shallow-water layer represents the mass above a given isentrope in the mid-troposphere. Since atmospheres are stably stratified, the entropy increases with height, and thus radiative heating (which increases the entropy of air) transports mass upward across isentropes, while radiative cooling (which decreases the entropy of air) transports mass downward across isentropes. Thus, day-night heating in the shallow-water system is parameterized as a mass source/sink that adds mass on the dayside (representing its transport into the upper-tropospheric shallow-water layer from the lower troposphere) and removes it on the nightside (representing its transport from the shallow-water layer into the lower troposphere). Here, we represent this process as a Newtonian relaxation with a characteristic radiative timescale, $\tau_{\text {rad }}$, that is a specified free parameter. The models are identical to those in Showman et al. (2013) except that the planetary parameters are chosen to be appropriate to a terrestrial exoplanet.

Figure 17 demonstrates that the amplitude of the daynight forcing exerts a major effect on the resulting circulation patterns. The four simulations are identical except for the imposed radiative time constant, which ranges from short in the top panels to long in the bottom panels. When the radiative time constant is extremely short (Figure $17 \mathrm{p}$ ), the circulation consists primarily of day-night flow in the upper troposphere, and the thermal structure is close to radiative equilibrium, with a hot dayside and a cold nightside. The radiative damping is so strong that any globalscale waves of the sort shown in Figure 16 are damped out. This simulation represents a complete breakdown of the WTG regime described in Section 2.2.2 Intermediate radiative time constants (Figure $17 \mathrm{p}$ and c) lead to large day-night contrasts coexisting with significant dynamical structure, including planetary scale waves that drive a superrotating equatorial jet. When the radiative time constant is long (Figure 17d), the circulation exhibits a zonally banded pattern, with little variation of the thermal structure in longitude-despite the day-night nature of the imposed thermal forcing. In this limit, the circulation is firmly in the WTG regime. Interestingly, the day-night forcing is sufficiently weak that superrotation does not occur in this case.

In a planetary atmosphere, the radiative time constant should increase with increasing temperature or decreasing atmospheric mass. Figure 17 therefore suggests that synchronously rotating planets that are particularly hot, or have thin atmospheres, may exhibit day-to-night flow patterns with large day-night temperature differences, while planets that are cooler, or have thicker atmospheres, may exhibit banded flow patterns with smaller day-night temperature differences (e.g., Showman et al. 2013). In the context of hot Jupiters, these arguments suggest that the most strongly irradiated planets should exhibit less efficient daynight heat redistribution (with temperatures closer to radiative equilibrium) than less-irradiated planets, a trend that already appears to be emerging in secondary-eclipse observations (Cowan and Agol 2011).

In the context of terrestrial planets, the trend in Figure 17 could likewise describe a transition in circulation regime occurring as a function of incident stellar flux, but it might also describe a transition as a function of atmospheric mass for a given stellar flux. Because of its thin atmosphere, Mars, example, has a radiative time constant considerably shorter than that on Earth, which helps to explain the much larger day-night temperature differences on Mars compared to Earth. Even more extreme is Jupiter's moon Io, due to its particularly tenuous atmosphere (surface pressure $\sim$ nanobar). $\mathrm{SO}_{2}$ is liberated by sublimation on the dayside but collapses into surface frost on the nightside, and the resulting day-night pressure differences are thought to drive supersonic flows from day to night (Ingersoll et al. 1985; Ingersoll 1989) ${ }^{13}$ In the exoplanet context, close-in rocky planets subject to extreme stellar irradiation may lie in a similar regime, with tenuous atmospheres maintained by sublimating silicate rock on the dayside, fast day-night airflows, and condensation of this atmospheric material on the nightside (Léger et al. 2011; Castan and Menou 2011). Prominent examples include CoRoT-7b, Kepler-10b, and 55 Cnc-e. Even terrestrial exoplanets with temperatures similar to those of Earth or Mars could experience sufficiently large day-night temperature differences to cause freeze-out of the atmosphere on the nightside if the atmosphere is particularly thin (Joshi et al. 1997), an issue we return to in Section 4

\section{HYDROLOGICAL CYCLE}

The hydrological cycle-broadly defined, where the condensate may be water or other chemical species-plays a fundamental role in determining the surface climate of a planet. It directly influences the surface climate by setting

\footnotetext{
${ }^{13}$ Neither Mars nor Io is synchronously rotating with respect to the Sun, but one can still obtain large day-night temperature differences in such a case if the radiative time constant is not long compared to the solar day.
} 

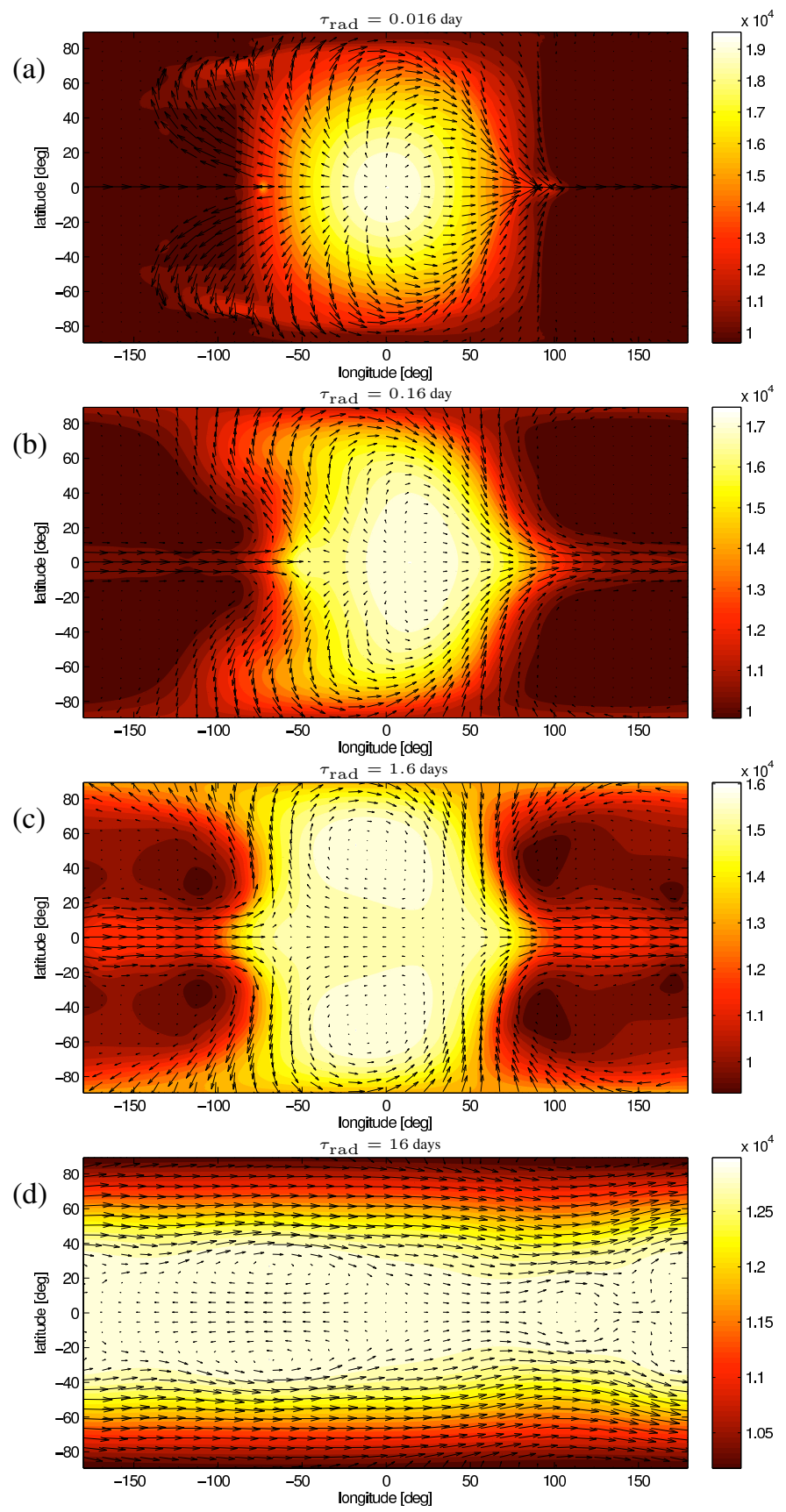

Fig. 17.- Layer thickness $g h$ (orange scale, units $\mathrm{m}^{2} \mathrm{~s}^{-2}$ ) and winds (arrows) for the equilibrated (steady-state) solutions to the shallow-water equations in full spherical geometry for sychronously rotating terrestrial exoplanets driven by a daynight thermal forcing. Specifically, to drive the flow, a mass source/sink term $\left(h_{\mathrm{eq}}-h\right) / \tau_{\mathrm{rad}}$ is included in the continuity equation, where $h_{\mathrm{eq}}$ is a specified radiative-equilibrium layer thickness, $h$ is the actual layer thickness (depending on longitude $\lambda$, latitude $\phi$, and time), and $\tau_{\text {rad }}$ is a specified radiative time constant. Here $h_{\text {eq }}$ equals a constant, $H$, on the nightside, and equals $H+\cos \lambda \cos \phi$ on the dayside. The substellar point is at longitude, latitude $\left(0^{\circ}, 0^{\circ}\right)$ (for further details about the model, see Showman et al. 2013). Here, the planet radius is that of Earth, rotation period is 3.5 Earth days, and $g H=10^{4} \mathrm{~m}^{2} \mathrm{~s}^{-2}$. These values imply an equatorial deformation radius, $(\sqrt{g H} / \beta)^{1 / 2}$, which is $60 \%$ of the planetary radius. The four models are identical in all ways except the radiative time constant, which is varied from 0.016 to 16 Earth days from top to bottom, respectively. When the radiative time constant is short, the flow exhibits a predominantly day-night circulation pattern with a large day-night contrast close to radiative equilibrium (a). Intermediate radiative time constants (b and c) still exhibit considerable day-night contrasts, yet allow the emergence of significant zonal flows. Long radiative time constants (d) lead to a zonally banded flow pattern with little variation of thermal structure in longitudedespite the day-night forcing. 
the spatial and temporal distributions of precipitation and evaporation and indirectly influences the surface temperature by affecting the horizontal energy transport, vertical convective fluxes, atmospheric lapse rate, and planetary albedo; these are key aspects of planetary habitability. The hydrological cycle is also fundamental in determining the radiative balance of the planet through the humidity and cloud distributions. The planetary radiative balance both affects the surface climate and is what is remotely observed.

Here, we survey the role of the hydrological cycle in affecting the atmospheric circulation and climate of terrestrial planets. We begin with a brief discussion of the role of the ocean (Section 3.1), as this is the ultimate source of atmospheric moisture and can influence atmospheric circulation in a variety of ways. We next discuss the thermodynamics of phase change (Section 3.2), which has significant implications for how a hydrological cycle affects the circulation. Global precipitation is discussed next (Section 3.3), with an emphasis on energetic constraints that are independent of details of the atmospheric circulation. The following section (3.4) highlights the important role of the atmospheric circulation for regional precipitation, in contrast to global precipitation. We then follow with a survey of how the atmospheric circulation helps to control the distribution of humidity (Section 3.5) and clouds (Section 3.6). Lastly, we summarize how the hydrological cycle in turn affects the structure of the atmospheric circulation (Section 3.7).

\subsection{Oceans}

The presence of an ocean affects planetary climate and atmospheric circulations in several key ways. We begin the discussion of the hydrological cycle with the ocean in recognition that it is the source of water vapor in an atmosphere, and the influences of the hydrological cycle on climate (presented in what follows) may be modulated by differences in water availability on other planets. Oceanography is, of course, a vast field of research, and we will only briefly discuss aspects that may be of immediate interest in the exoplanet context. For overviews of physical oceanography and the ocean's effect on climate in the terrestrial context, see for example Peixoto and Oort (1992), Siedler et al. (2001), Pedlosky (2004), Vallis (2006, 2011), Marshall and Plumb (2007), Olbers et al. (2011), and Williams and Follows (2011), among others.

Oceans on exoplanets will span a wide range. At one extreme are planets lacking surface condensate reservoirs (Venus) or with liquid surface reservoirs small enough to form only disconnected lakes (Titan). In such a case, the surface condensate should not contribute significantly to the meridional or day-night heat transport, but could still significantly impact the climate via energy exchanges and evaporation into the atmosphere. At the other extreme are super Earths whose densities are sufficiently low to require significant fractions of volatile materials in their interiors. Prominent examples include GJ 1214b (Charbonneau et al. 2009; Rogers and Seager 2010; Nettelmann et al. 2011) and sev- eral planets in the Kepler-11 system (Lissauer et al. 2011, Lopez et al. 2012); many of the hundreds of super Earths discovered by Kepler also likely fall into this category (e.g., Rogers et al. 2011). Many such planets will contain waterrich fluid envelopes thousands of km thick. If their interior temperature profiles are sufficiently hot (in particular, if, at the pressure of the critical point, the atmospheric temperature exceeds the temperature of the critical poin ${ }^{14}$, then these planets will exhibit a continuous transition from a supercritical fluid in the interior to a gas in the atmosphere, with no phase boundary; on the other hand, if their interior temperature profiles are sufficiently cold, then their waterrich envelopes will be capped by an oceanic interface, overlain by an atmosphere (Léger et al. 2004; Wiktorowicz and Ingersoll 2007). Earth lies at intermediate point on this continuum, with an ocean that is a small fraction of the planet's mass and radius, yet is continuously interconnected and thick enough to cover most of the surface area.

Our understanding of ocean dynamics and oceanatmosphere interactions stem primarily from Earth's ocean, so to provide context we first briefly overview the ocean structure. The Earth's oceans have a mean thickness of $3.7 \mathrm{~km}$ and a total mass $\sim 260$ times that of the atmosphere. At the surface, the ocean is warm at the equator $\left(\sim 27^{\circ} \mathrm{C}\right.$ in an annual and longitudinal average) and cold at the poles $\left(\sim 0^{\circ} \mathrm{C}\right)$. The deep ocean temperature is relatively uniform at a temperature of $\sim 1-2^{\circ} \mathrm{C}$, thoughout the world-even in the tropics. The transition from warmer surface water to cooler deep water is called the thermocline and occurs at $\sim 0.5 \mathrm{~km}$ depth, depending on latitude. Because low-density, warmer water generally lies atop high-density, cooler water, the ocean is stably stratified and does not convect except at a few localized regions near the poles. However, turbulence caused by wind and waves homogenizes the top $\sim 10-100 \mathrm{~m}$ (depending on weather conditions and latitude), leading to profiles of density, salinity, and composition that vary little across this so-called "mixed layer." Because of its efficient communication with the atmosphere, the thickness of the mixed layer exerts a strong effect on climate (e.g., on the extent to which the ocean modulates seasonal cycles). Nevertheless, the deeper ocean also interacts with the atmosphere on a wide range of timescales up to thousands of years.

The Earth's oceans affect the climate in numerous ways. For discussion, it is useful to decompose the role of the ocean into time-dependent (e.g., modification of heat capacity) and time-independent (e.g., time-mean energy transports) categories.

Consider the time-dependent energy budget of the ocean mixed layer:

$$
\alpha \frac{\partial T_{\text {surf }}}{\partial t}=R_{\text {sfc }}-L E-S H-\nabla \cdot F_{o},
$$

\footnotetext{
${ }^{14}$ For a pure water system, the critical point pressure and temperature are 221 bars and $647 \mathrm{~K}$ respectively, but they depend on composition for a system containing other components such as hydrogen (e.g., Wiktorowicz and Ingersoll 2007).
} 
where $\alpha$ is the heat capacity per unit area of the mixed layer (approximately equal to $\rho c_{p} h$, where $\rho$ is the density, $c_{p}$ is the specific heat, and $h$ is the thickness of the mixed layer), net surface radiative flux $R_{\mathrm{sfc}}$, latent enthalpy flux $L E$, sensible enthalpy flux $S H$, and ocean energy flux divergence $\nabla \cdot F_{o}$, including vertical advection into the base of the mixed layer. Earth's mixed layer depth has geographic and seasonal structure because it is connected both to the interior ocean's thermal structure and currents from below and is forced by the atmosphere and radiation from above.

From this budget, a clear time-dependent effect of the ocean is that it acts as a thermal surface reservoir, which reduces the seasonal and diurnal cycle amplitude compared to possible land-covered planets ( $\alpha$ for the ocean is substantially larger than for land; Hartmann 1994a; Pierrehumbert 2010). In addition to the direct modification of the heat capacity, the presence of an ocean leads to weaker temperature fluctuations through evaporation, which is a more sensitive function of temperature than surface longwave radiation or sensible surface fluxes (e.g., Pierrehumbert 2010). Oceans further influence a planet's thermal evolution on long timescales through the storage of heat in the interior ocean (i.e., the vertical component of the ocean energy flux divergence in Eq. 23) and through their role as a chemical reservoir (Sarmiento and Gruber 2006); the ocean storage and release of carbon is important for Earth's glacial cycles, for example.

In addition to the effect of the ocean in determining a planet's time-dependent evolution, oceans modify a planet's time-mean climate. Evaporation of water from the ocean is the essential moisture source to the atmosphere and to land regions with net precipitation. Oceans affect the radiation balance of a planet directly through their albedo and indirectly through providing the moisture source for the atmospheric hydrological cycle. Finally, ocean energy transport is important in determining the surface temperature.

Many authors have examined the sensitivity of ocean energy transport to changes in surface wind stress and surface buoyancy gradients, often decomposing the energy transport into components associated with deep meridional overturning circulations and shallow, wind-driven circulations (cf. Ferrari and Ferreira 2011). Here, we note that ocean energy transport can affect surface climate differently than atmospheric energy transport. For example, Enderton and Marshall (2009) presented simulations with different simplified ocean basin geometries; the ocean energy transport, surface temperature, and sea ice of the simulated equilibrium climates differed, but the total energy transport was relatively unchanged (i.e., the changes in atmospheric energy transport largely offset those of the ocean ${ }^{15}$. An implication of these results is that while observations of a planet's top-of-atmosphere radiation allow the total (ocean and atmosphere) energy transport to be determined, this alone is not sufficient to constrain the surface temperature gradient.

${ }^{15}$ Changes in atmospheric and oceanic energy transport need not compensate in general (e.g., Vallis and Farneti|2009|

\subsection{Thermodynamics of phase change}

Before describing the ways the hydrological cycle can interact with the atmospheric dynamics (which we do in subsequent subsections), we first summarize the pure thermodynamics of a system exhibiting phase changes. Most atmospheres in the Solar System have contituents that can condense: water on Earth, $\mathrm{CO}_{2}$ on Mars, methane on Titan, $\mathrm{N}_{2}$ on Triton and Pluto, and several species, including $\mathrm{H}_{2} \mathrm{O}, \mathrm{NH}_{3}$, and $\mathrm{H}_{2} \mathrm{~S}$, on Jupiter, Saturn, Uranus, and Neptune. Because atmospheric air parcels change temperature and pressure over time (due both to atmospheric motion and to day-night or seasonal temperature swings), air parcels make large excursions across the phase diagram, and often strike one or more phase boundaries-leading to condensation. This condensation can exert major influence over the circulation.

The starting point for understanding these phase changes is the Clausius-Clapeyron equation, which relates changes in the saturation vapor pressure of a condensable vapor, $e_{s}$, to the latent heat of vaporization $L$, temperature $T$, density of vapor $\rho_{\text {vap }}$, and density of condensate $\rho_{\text {cond }}$

$$
\frac{d e_{s}}{d T}=\frac{1}{T} \frac{L}{\rho_{\text {vap }}^{-1}-\rho_{\text {cond }}^{-1}} .
$$

For condensate-vapor interactions far from the critical point, the condensate density greatly exceeds the vapor density and can be ignored in Eq. 24). Adopting the ideal-gas law, the equation can then be expressed

$$
\frac{1}{e_{s}} \frac{d e_{s}}{d T}=\frac{L}{R_{v} T^{2}}
$$

where $R_{v}$ is the gas constant of the condensable vapor.

The saturation vapor pressure is the pressure of vapor if the air were in equilibrium with a saturated surface; it increases with temperature and is a purely thermodynamic quantity. In contrast, the vapor pressure of an atmosphere is not solely a thermodynamic quantity, as atmospheres are generally subsaturated. Therefore, the vapor pressure depends on the relative humidity $\mathcal{H}=e / e_{s}$, which in turn depends on the atmospheric circulation (discussed in what follows). Condensation occurs when air becomes supersaturated, although there are also microphysical constraints such as the availability of condensation nuclei. A consequence of 25) is that atmospheric humidity will increase with temperature, if relative humidity is unchanged.

The vertical structure of water vapor or other condensible species can be quantified by comparing it to that of pressure. The pressure scale height $H_{p}$ for hydrostatic atmospheres is given by

$$
\frac{1}{H_{p}}=\frac{\partial \ln p}{\partial z}=\frac{g}{R T} .
$$

For Earth, $g \sim 10 \mathrm{~m} \mathrm{~s}^{-2}, T \sim 280 \mathrm{~K}, R \sim 300 \mathrm{~J} \mathrm{~kg}^{-1} \mathrm{~K}^{-1}$, the pressure scale height $H_{p} \approx 8 \mathrm{~km}$. The pressure scale height is a decreasing function of planet mass (through $g$ ) and an increasing function of planet temperature. 
Because the saturation vapor pressure of condensable species depend strongly on temperature, it is possible for the scale height of water-and other condensables - to differ greatly from the pressure scale height. The scale height of water vapor $H_{w}$ can be estimated as follows

$$
\frac{1}{H_{w}}=\frac{\partial \ln e_{s}}{\partial z}=\frac{\partial \ln e_{s}}{\partial T} \frac{\partial T}{\partial z}=\frac{L}{R_{v} T^{2}} \frac{\partial T}{\partial z},
$$

where the chain rule and Clausius-Clapeyron relationship (25) have been used and vertical variations in relative humidity have been neglected. The scale height of water vapor is the inverse of the product of the lapse rate $\Gamma=-\partial T / \partial z$ and the fractional change of vapor pressure with temperature (i.e., the Clausius-Clapeyron relationship). The scale height of water increases with temperature [through [25] and with decreasing lapse rate (i.e., less rapid vertical temperature decrease).

Using Earth-like values in (26) and 27) of $g \sim 10 \mathrm{~m} \mathrm{~s}^{-2}$, $T \sim 280 \mathrm{~K}, \quad R \sim 300 \mathrm{~J} \mathrm{~kg}^{-1} \mathrm{~K}^{-1}, \quad R_{v} \sim 450 \mathrm{~J} \mathrm{~kg}^{-1} \mathrm{~K}^{-1}$, $L \sim 2.5 \times 10^{6} \mathrm{~J} \mathrm{~kg}^{-1}$, the ratio of the water vapor and pressure scale heights $H_{w} / H_{p}$ is $\sim 0.2$ for dry adiabatic lapse rate $\left(\Gamma=g / c_{p}\right)$. Thus, for Earth-like situations, the scale height of water vapor is significantly less than the pressure scale height. This implies that near-surface water vapor dominates the column water vapor (the mass-weighted vertical integral of water vapor from the surface to the top of atmosphere). Moreover, the saturation vapor pressure at the tropical tropopause is $\sim 10^{4}$ times less than at the surface. As a result, the middle and upper atmosphere (stratosphere and above) are very dry. Still, that the bulk of the atmospheric water vapor resides near the surface does not mean that the upper tropospheric humidity is unimportant: small concentrations there can be important in affecting the radiation balance as water vapor is a greenhouse gas (e.g., Held and Soden 2000); moreover, upper-tropospheric water controls the formation and distribution of cirrus cloud, which is important in the atmospheric radiation balance.

The condensable gas will be a larger fraction of the pressure scale height if the latent heat is smaller or if the tropospheric stratification is greater. Titan provides an example in our solar system; the methane abundance at the tropopause is only a factor of $\sim 3$ smaller than near the surface (Niemann et al. 2005). In such a situation, the condensable vapor abundance in the stratosphere may be considerable, which can have implications for planetary evolution because it will affect the rate at which such species (whether water or methane) can be irreversibly lost via photolytic breakup and escape of hydrogen to space.

\subsection{Global Precipitation}

A planet's precipitation is a fundamental part of its hydrological cycle and has important implications for global climate feedbacks such as the carbonate-silicate feedback cycle (Section 4). At equilibrium, a planet's precipitation equals its evaporation in the global mean. Global precipitation is controlled by energy balance requirements, either of the surface (to constrain evaporation) or of the atmospheric column (to constrain the latent heating from precipitation). More complete discussions and reviews of the control of global precipitation can be found in Held (2000), Pierrehumbert (2002), Allen and Ingram (2002), Schneider et al. (2010), and O'Gorman et al. (2011).

The dry static energy $s=c_{p} T+g z$ is the sum of the specific enthalpy and potential energy. The dry static energy is materially conserved in hydrostatic atmospheres in the absence of diabatic processes (e.g., radiation or latent heat release), if the kinetic energy of the atmosphere is neglected (cf. Betts 1974; Peixoto and Oort 1992). The dry static energy budget is

$$
\frac{\partial s}{\partial t}+\mathbf{v} \cdot \nabla s=Q_{r}+Q_{c}+S H
$$

where $Q_{r}$ is the radiative component of the diabatic tendency, $Q_{c}$ is the latent-heat release component of the diabatic tendency, and $S H$ is the sensible component of the surface enthalpy flux. Taking the time- and global-mean of the dry static energy budget eliminates the left-hand side of 28 , so that when it is integrated vertically over the atmospheric column, the global-mean precipitation is a function of the radiation and sensible surface flux

$$
L\langle P\rangle=\left\langle R_{a t m}\right\rangle+\langle S H\rangle=\left\langle R_{T O A}\right\rangle-\left\langle R_{s f c}\right\rangle+\langle S H\rangle,
$$

with latent heat of vaporization $L$, precipitation $P$, net atmospheric radiation $R_{a t m}$, net top-of-atmosphere (TOA) radiation $R_{T O A}$, and net surface radiation $R_{s f c}$. The operator $\langle\cdot\rangle$ denotes the time- and global-mean. The radiation terms are evaluated only at the surface and top-ofatmosphere because of the vertical integration over the atmospheric column, and they can be further decomposed into components associated with the shortwave and longwave parts of the radiation spectrum.

The time- and global-mean of the surface energy budget (23) is

$$
L\langle E\rangle=\left\langle R_{s f c}\right\rangle-\langle S H\rangle,
$$

with evaporation $E$ and other variables defined as in 29. In both budgets, the sensible turbulent surface flux (the dry component of the surface enthalpy flux) enters, so the global precipitation is not solely a function of the radiative properties of the atmosphere. The turbulent surface fluxes are represented by bulk aerodynamic formula in GCMs (Garratt 1994) [e.g., $S H=\rho c_{d}\left\|\mathbf{v}_{\text {surf }}\right\|\left(T_{s}-T_{a}\right)$ ], which depend on the temperature difference between the surface and surface air $\left(T_{s}-T_{a}\right)$, surface wind speed $\left\|\mathbf{v}_{\text {surf }}\right\|$, and surface roughness (through $c_{d}$ ).

From these budgets, one expects global precipitation to increase if the solar constant increases (Fig. 18p), the top-of-atmosphere or surface albedo decreases, or sensible surface fluxes decrease. Reductions in longwave radiation at the surface that are greater than those at the top-ofatmosphere will increase the global precipitation (Fig. 18), as is expected for increased greenhouse gas concentrations 
on Earth. This illustrates that the vertical structure of radiative changes is important. Another example is that changes in TOA albedo and surface albedo differ in their effect on global mean precipitation because they differ in their effect on shortwave absorption by the atmosphere [a component of $R_{a t m}$ in (4)]. Thus, it is not possible to infer globalmean precipitation from measurements of the radiances at the top-of-atmosphere $R_{T O A}$ because the sensible surface flux $S H$ and net surface radiation $R_{s f c}$ matter.

The surface energy budget suggests the existence of an approximate limit to the magnitude increases in globalmean precipitation due to increased greenhouse gas concentration. Under greenhouse gas warming, the air-sea temperature difference at the surface generally decreases. In the limit of no air-sea temperature difference, the net surface longwave radiation and sensible surface fluxes vanish, which leaves the cooling from latent surface fluxes to balance the warming from net solar radiation at the surface. This limit is approximately attained in idealized GCM simulations (Fig. 18a, O'Gorman and Schneider 2008). However, sensible surface fluxes may reverse sign (from cooling the surface to warming it), so the warmest simulation in Fig. 18 a exceeds the precipitation expected from all of the surface solar radiation being being converted into evaporation. The sensitivity of precipitation to greenhouse gas changes has implications for the rate of silicate weathering (see section 4) (Pierrehumbert 2002, Le Hir et al. 2009).

The energetic perspectives on global precipitation illustrates how the rate of change of global precipitation and atmospheric humidity can differ. If the relative humidity does not change, the atmospheric humidity increases with temperature at the rate given by the Clausius-Clapeyron relation (25). In contrast, the global precipitation depends on radiative fluxes such as the top-of-atmosphere insolation and longwave radiation. The radiative fluxes depend in part on the atmospheric humidity through water vapor's absorption of shortwave and longwave radiation (which gives rise to the water vapor feedback), but this dependence does not constrain the radiative changes to vary with the water vapor concentration at the rate given by 25. Precipitation and humidity have different dimension, as well: precipitation and evaporation are fluxes of water, whereas the atmospheric humidity is a concentration of water. The suggestion that precipitation changes in proportion to atmospheric humidity has been termed the "saturation fallacy" (Pierrehumbert et al.2007).

Another result that can be understood by considering the energy budget is that there is not a unique relationship between the global mean temperature and precipitation. For example, in geoengineering schemes that offset warming due to increased $\mathrm{CO}_{2}$ by increasing albedo (reducing the solar radiation), two climates with the same global mean temperature may have differing global mean precipitation because the sensitivity of global precipitation per degree temperature change are not equal for different types of radiation changes (Bala et al.2008, O'Gorman et al.2011). Figure 18 shows that warming forced by solar constant changes results in a larger increase in global-mean precipitation than warming forced by changes in the longwave optical depth in an idealized GCM (O'Gorman et al. 2011).

While the global mean precipitation is potentially a variable of interest in characterizing a planet's habitability, regional precipitation variations are substantial. Earth has regions of dry deserts and rainy tropical regions, where the annual-mean precipitation differs by a factor of $\sim 20$. The regional precipitation is far from the global mean because of water vapor transports by the atmospheric circulation. Furthermore, the sensitivity of regional precipitation to external perturbations can be of opposite sign as the global mean changes (for example, some regions may dry as the global mean precipitation increases Chou and Neelin 2004, Held and Soden 2006).

\subsection{Regional Precipitation}

To understand the variations in regional precipitation, we first illustrate the processes that give rise to condensation (Fig. 19. In tropical latitudes of Earth-like planets, there are time-mean regions of ascent where moist air is continually brought from the surface to the colder upper troposphere which leads to supersaturation and condensation (near the equator in Fig. 19). There are time-mean regions of subsidence where dry air from the upper troposphere descends toward the surface, so the mean circulation leads to subsaturation and precipitation occurs during transient events, when it does occur (near $30^{\circ}$ in Fig. 19 corresponding to the descending branches of the Hadley cells). In the extratropical regions of Earth-like planets, air parcels approximately follow surfaces of constant entropy which slope upward and poleward. As parcels ascend in the warm, poleward moving branches of extratropical cyclones (i.e., transient baroclinic eddies like those described in Section 2.1.2, they become supersaturated and condense in the colder regions of the atmosphere. This is illustrated in Fig. 19. where gray lines indicate entropy surfaces and black arrows indicate the trajectories of air masses. This description is focused on mean meridional circulations in the tropics and transient eddies in the extratropics. Superimposed on these processes affecting the zonal-mean precipitation are stationary eddies (that is, eddy structures that are fixed rather than traveling in longitude) or zonally asymmetric mean flows that give rise to important longitudinal variations in precipitation on Earth.

While the zonal mean is an excellent starting point for Earth's climate and atmospheric circulations, stationary eddies or zonally asymmetric mean flows are of central importance for tidally locked planets. The mean circulation aspect of the description will have an east-west component: the near-surface branch of the circulation will flow from the night side to the day side, ascend and precipitate there, and return to the night side aloft (Merlis and Schneider 2010). One can rotate the time-mean circulation in Fig. 19 from a north-south orientation (Hadley circulation) to an east-west orientation (Walker circulation) to conceptualize the effect 

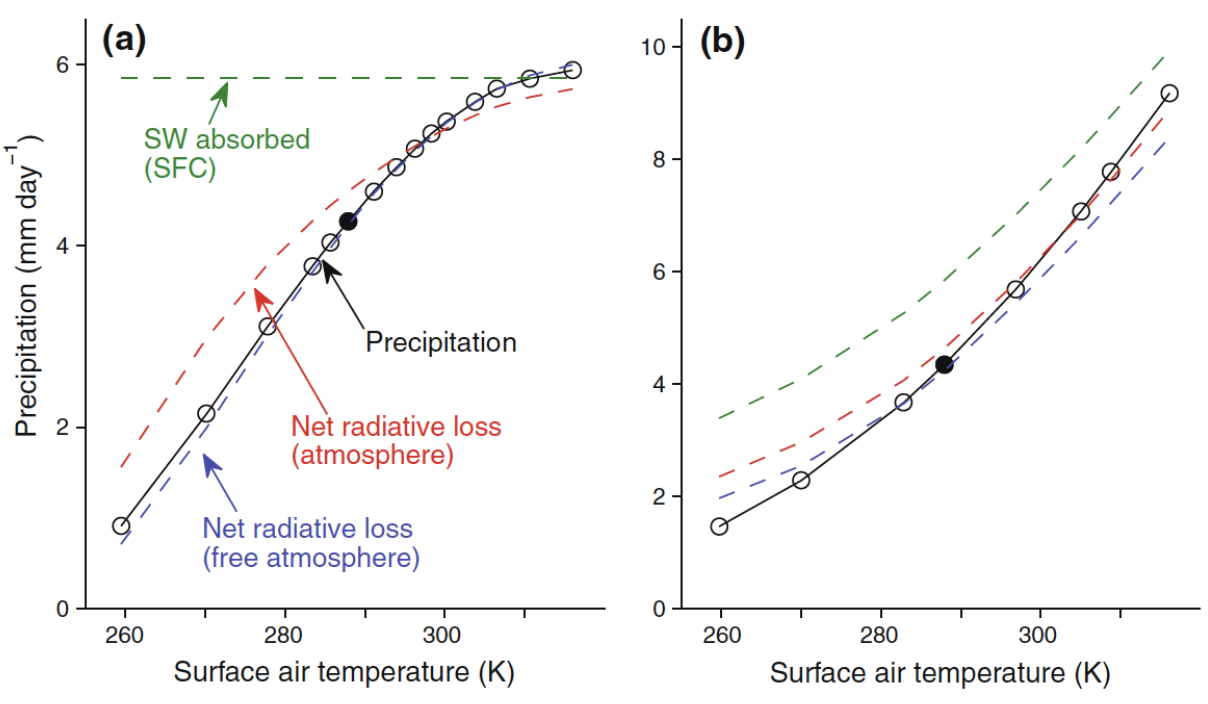

Fig. 18. - From O'Gorman et al. (2011): Global-mean precipitation (solid line with circles) versus global-mean surface air temperature in two series of statistical-equilibrium simulations with an idealized GCM in which (a) the optical depth of the longwave absorber is varied and (b) the solar constant is varied (from about $800 \mathrm{~W} \mathrm{~m}^{-2}$ to about $2300 \mathrm{~W} \mathrm{~m}^{-2}$ ). The filled circles indicate the reference simulation (common to both series) which has the climate most similar to present-day Earth's. The red dashed lines show the net radiative loss of the atmosphere, the blue dashed lines show the net radiative loss of the free atmosphere (above $\sigma=p / p_{s}=0.86$ ), and the green dashed lines show the net absorbed solar radiation at the surface (all in equivalent precipitation units of $\mathrm{mm}_{\text {day }}{ }^{-1}$ ).

of the night-side to day-side circulations on precipitation; however, the factors controlling the circulations themselves may differ as their angular momentum balances differ (the extent to which the circulation strength is slaved to the momentum balance or responds directly to radiation). In contrast, transient eddies (that is, eddies that travel in longitude) exist because temperature gradients are not aligned with the planet's spin axis, which gives rise to baroclinic instability (Section 2.1.2), so the conceptual picture cannot simply be rotated. The affect of zonal asymmetry of background state on baroclinic eddies is not settled; in particular, the extent to which stationary eddies and baroclinic eddies are separable (i.e., the extent to which they are linear) is a research area (Held et al.|2002, Kaspi and Schneider 2011).

Simulations of Earth-like tidally locked exoplanets from Merlis and Schneider (2010) illustrate the importance of zonally asymmetric (east-west) mean atmospheric circulations in determining precipitation (Fig. 20). For both slowly and rapidly rotating simulations, the surface zonal wind converges $\left[\partial_{x}[u]<0\right.$, with the $[\cdot]$ indicating a time-mean] on the day-side near the subsolar point, ascends, and diverges $\left(\partial_{x}[u]>0\right)$ in the upper troposphere. The night side features convergent mean zonal winds in the upper troposphere, subsidence, and divergent mean zonal winds near the surface. These are thermally direct circulations that transport energetic air from the day side to the night side. The water vapor is converging near the surface on the day side where there is substantial precipitation. There is a clear connection between the pattern of convergence in the surface winds and precipitation - the rapidly rotating case, in particular, has a crescent-shaped precipitation field. The shape of the convergence zone is similar to that of the Gill model (Gill 1980: Merlis and Schneider 2010).

To be quantitative about regional precipitation, consideration of the water vapor budget, a conservation equation for the atmospheric humidity, can be useful. For timescales long enough that the humidity tendency is small, the timemean (denoted $[\cdot]$ ) net precipitation, $[P-E]$, is balanced by the mass-weighted vertical integral (denoted $\{\cdot\}$ ) of the convergence of the water vapor flux, $-\nabla \cdot[\mathbf{u} q]$ :

$$
[P-E]=-\int_{0}^{p_{s}} \nabla \cdot[\mathbf{u} q] \frac{\mathrm{d} p}{g}=-\nabla \cdot\{[\mathbf{u} q]\},
$$

with horizontal wind vector $\mathbf{u}$ and specific humidity $q$. The atmospheric circulation converges water vapor into regions of net precipitation $(P>E)$ from regions of net evaporation $(E>P)$, where there is a divergence of water vapor. The water vapor budget makes explicit that the atmospheric circulation is fundamental in determining this basic aspect of climate.

The small scale height of water vapor in Earth-like atmospheres emphasizes the near-surface water vapor concentration, so a conceptually useful approximation to (31) is to consider the near-surface region of the atmosphere. For example, the precipitation distribution follows the pattern of the convergence of the surface wind in Fig. 20

Note that the surface temperature does not directly appear in (31). In spite of this, there have been many attempts to relate precipitation to surface temperature for Earth's 


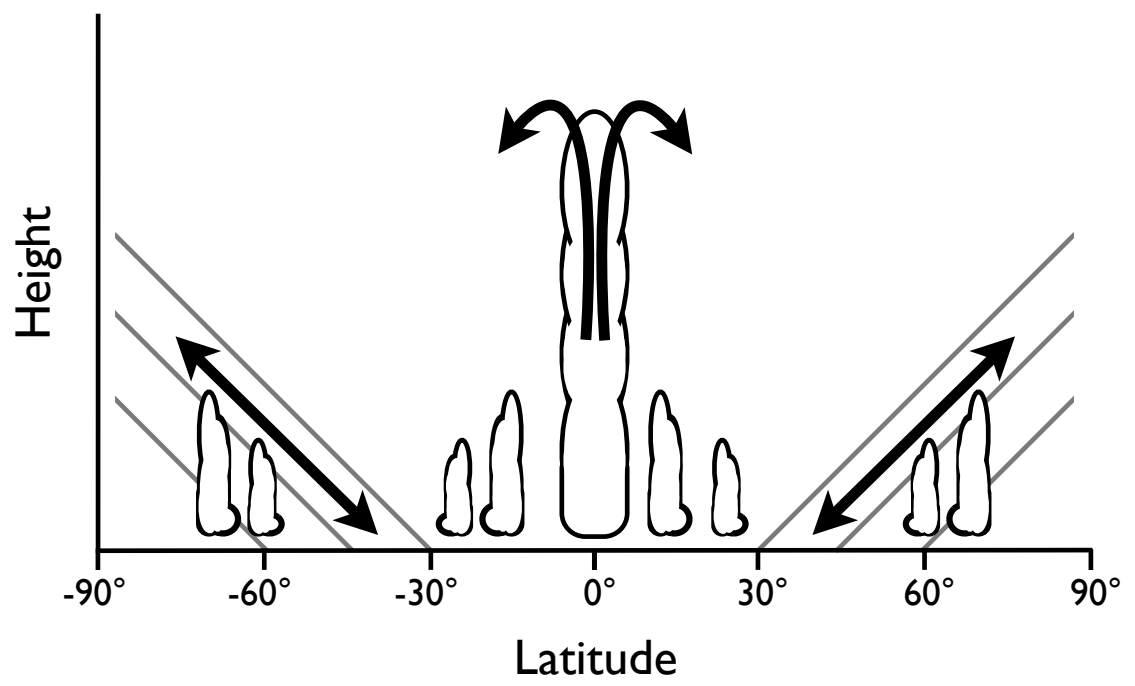

Fig. 19. - Processes leading to condensation and affecting atmospheric humidity for Earth-like planets, based on Figure 8 of Held and Soden (2000). Gray lines indicate surfaces of constant entropy which air parcels approximately follow. In the tropics, the ascending branch of the Hadley cell leads to significant condensation and rainfall, whereas the descending branches (at latitudes of $\sim 20-30^{\circ}$ on Earth) exhibit less condensation and fewer clouds. In the extratropics, the rising motion in baroclinic eddies transporting energy poleward and upward (indicated schematically with sloping lines) leads to significant condensation.

climate. The surface temperature may vary together with aspects of climate that do directly determine the amount of precipitation (e.g., high near-surface humidity or the largest magnitude near-surface convergence of the horizontal winds may occur in the warmest regions of the tropics). There are conceptual models that relate the surface temperature to pressure gradients that determine regions of convergence through the momentum balance (Lindzen and Nigam 1987; Back and Bretherton 2009a). Likewise, parcel stability considerations (e.g., where is there more convective available potential energy) may be approximately related to regions of anomalously high surface temperature (Sobel 2007; Back and Bretherton 2009b).

\subsection{Relative humidity}

Reviews by Pierrehumbert et al. (2007) and Sherwood et al. (2010b) discuss the processes affecting Earth's relative humidity and its sensitivity to climate changes.

The atmosphere of a planet is generally subsaturatedthe relative humidity is less than $100 \%$. The relative humidity of the atmosphere depends on the combined effect of inhomogeneities in temperature and the atmospheric circulation.

For Earth-like planets, the atmospheric circulation produces dry air by advecting air upwards and/or polewards to cold temperatures where the air becomes supersaturated and water vapor condenses (Fig. 19). When this air is advected downward or equatorward, the water vapor concentration will be subsaturated with respect to the local temperature.

The nonlocal influence of the temperature field on the atmospheric humidity through the atmospheric circulation can be formalized by the tracer of last saturation paradigm (Galewsky et al. 2005, Pierrehumbert et al. 2007). In this framework, the humidity of a given region in the atmosphere is decomposed into the saturation humidity at the non-local (colder) region of last saturation, with weights given by the probability that last saturation occurred there.

In addition to the adiabatic advection, convection affects the relative humidity and can act to moisten or dry the atmosphere (e.g., Emanuel 1994). In subsiding regions of Earth's tropics, convection moistens the free troposphere to offset the substantial drying associated with subsidence from the cold and dry upper troposphere (e.g., Couhert et al. 2010). Therefore, both the water vapor advection by the large-scale circulations and small-scale convective processes, with the latter parameterized in GCMs, are important in determining the relative humidity.

These relative humidity dynamics are important for various aspects of planetary atmospheres: they influence the radiation balance, atmospheric circulations, and precipitation. 

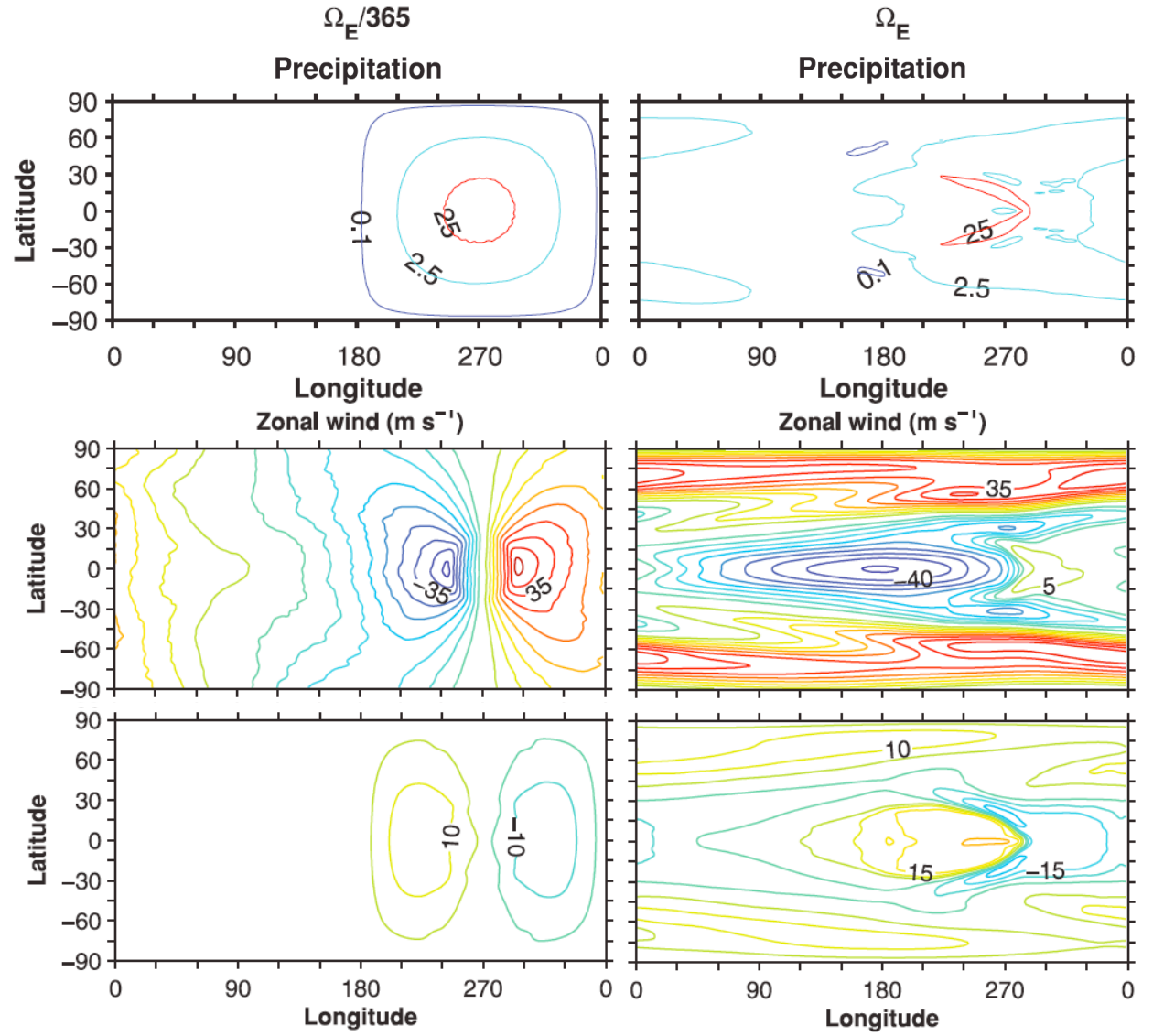

Fig. 20.- Time-mean circulation in two GCM simulations of Earth-like synchronously rotating exoplanets; models with rotation periods of one Earth year and one Earth day, respectively, are shown on the left and right. Top row shows timemean precipitation (contours of $0.1,2.5$, and $25.0 \mathrm{~mm}$ day $^{-1}$ ). Middle and bottom rows show time-mean zonal wind on the $\sigma=p / p_{s}=0.28$ model level and at the surface, respectively (contour interval is $5 \mathrm{~m} \mathrm{~s}^{-1}$; the zero contour is not displayed). The subsolar point is fixed at $270^{\circ}$ longitude in both models. Adapted from Figs. 2,4,11 of Merlis and Schneider (2010).

The processes controlling the relative humidity are tied directly to three-dimensional atmospheric circulations, which is a difficulty of single column models. Single column models do not explicitly simulate the atmospheric eddies or mean circulations that advect water and set the global-mean relative humidity. Furthermore, spatial variations in relative humidity are radiatively important. For example, the same global-mean relative humidity with a different spatial distribution has a different global-mean radiative cooling (Pierrehumbert 1995; Pierrehumbert et al.|2007). Therefore, the magnitude the day-side to night-side relative humidity contrasts of tidally locked exoplanets may be important in determining the global climate.

For greenhouse gas-forced climate changes on Earth, relative humidity changes $\left(<1 \% \mathrm{~K}^{-1}\right)$ are typically smaller than specific humidity changes $\left(\sim 7 \% \mathrm{~K}^{-1}\right)$ (Held 2000 . Sherwood et al. 2010a). Therefore, it is a useful state variable for climate feedback analysis that avoids strongly canceling positive water vapor feedback and negative lapse rate feedback found in the conventional feedback analysis that uses specific humidity as a state variable (Held and Shell 2012). Climate feedback analysis depends on the mean state, which clearly differs between tidally locked exoplanets and Earth-like planets. A climate feedback analysis has not been performed for a tidally locked planet to diagnosing the relative contributions of the water vapor, lapse rate, albedo, and cloud feedbacks to the climate sensitivity.

\subsection{Clouds}

The formation of clouds from the phase change of condensible species-like water vapor-is often associated with ascending motion. Ascent may be the result of convergent horizontal winds or convective instability, so clouds typically have small space and time scales. Therefore, the spatial distribution of clouds and their sensitivity to external parameters such as the rotation rate or solar constant depend on atmospheric turbulence of a variety of scales: from the scales of the global circulations $(\sim 1000 \mathrm{~km})$ down to those of moist convection and boundary layer turbulence ( $\sim 10 \mathrm{~km}$ and smaller). 
Clouds can affect the radiation balance substantially. To illustrate, we consider an Earth-like case, although other possibilities exist (e.g., Wordsworth et al.2010). First, consider the longwave component of the radiation and assume that clouds are completely opaque in that region of the spectrum. If a cloud is low in the atmosphere (near the surface), the temperature at the top of the cloud, from where the radiation is re-emitted after absorption, will be close to the temperature at which the radiation would be emitted in the absence of any clouds (e.g., from the surface or the clear air near the surface). In this case, the cloud will not substantially affect the longwave radiation relative to cloud-free conditions. If a cloud is high in the atmosphere (near the tropopause, for example), the temperature at the top of the cloud will be substantially cooler than the emission temperature in the absence of clouds. So, high clouds enhance the atmospheric greenhouse by absorbing longwave radiation emitted at the higher temperatures of atmosphere and surface below and re-emitting longwave radiation at the colder cloud temperature. Simply characterizing the effect of clouds on the shortwave radiation depends more subtly on the optical characteristics. There can be a substantial reflection (e.g., cumulus or stratus) or little reflection (e.g., cirrus) of shortwave radiation. Additionally, the effect of clouds on the planetary albedo depends on the surface albedo; for example, in a Snowball-Earth state (Section 4.2, a planet may be largely ice covered, in which case clouds will not significantly modify the albedo. Considering all these effects, the net radiative effect of clouds (the sum of shortwave and longwave) can be warming, cooling, or close to neutral. In the current Earth climate, these three possibilities are manifest with cirrus, stratocumulus, and convecting cumulus clouds, respectively.

The cloud radiative forcing (the difference in top-ofatmosphere net radiation between cloudy and clear conditions) on Earth is negative: the Earth would be warmer without clouds. This does not imply that clouds are expected to damp (negative radiative feedback) rather than amplify (positive radiative feedback) radiative perturbations. In comprehensive general circulation model projections of climate change, the cloud feedback is on average positive (i.e., the change in cloud properties amplifies the radiative perturbation from $\mathrm{CO}_{2}$ changes) (Soden and Held 2006). However, there is substantial uncertainty in the magnitude, and the physical basis of this projection, if there is one, is still being elucidated (e.g., Bony et al. 2006, Zelinka and Hartmann 2010).

In addition to their important role in determining climate through the radiation balance, clouds likewise affect remote (e.g., space-based) observations of planets. Interpreting cloud observations may help reveal aspects of the atmospheric circulation, such as the characteristic space and timescales of a planet's "weather" (e.g., cloud tracking on Jupiter), and climate (e.g., clouds on Titan indicate regions of precipitation). Preliminary efforts are being made to explore the effects of clouds on lightcurves and spectra of terrestrial exoplanets (e.g. Pallé et al.|2008; Cowan et al.|2009,
Fujii et al. 2011; Robinson et al. 2011, Kawahara and Fujii 2011; Fujii and Kawahara 2012; Sanromá and Pallé 2012. Gómez-Leal et al.|2012; Karalidi et al. 2012).

\subsection{The hydrological cycle's effect on atmospheric temperature and circulations}

While much of our discussion has focused on the role of atmospheric circulations in shaping the distributions of precipitation, humidity, and clouds, water vapor is not a passive tracer and can affect the atmospheric temperature and winds ${ }^{16}$. Here, we outline some ways in which the latent heat release of condensation affects the atmosphere's thermal structure and water vapor influences the energetics of atmospheric circulations. Additional material may be found in reviews by Sobel (2007) and Schneider et al. (2010).

\subsubsection{Thermal structure}

The atmosphere's stratification in low latitudes (or slowly rotating regions more generally) is largely determined by convection. The tropical atmosphere is close to a moist adiabat on Earth (e.g., Xu and Emanuel 1989), although entrainment and ice-phase processes affect the upper troposphere (Romps and Kuang 2010). Simulations of Earth-like tidally locked planets (Merlis and Schneider 2010) also have near moist adiabatic stratification over large regions of the day side. The influence of convecting regions is nonlocal: gravity wave propagate away density variations (see Section 2.2.2), whereas gradients can be rotationally balanced in the extratropics (more generally, in regions where the Coriolis force is important in the horizontal momentum balance). The relative importance of moist convection in determining the tropical stratification will be mediated by the geography of condensate reservoirs (e.g., Titan or possible dry exoplanets) and the vertical distribution of radiatively active agents (e.g., Venus and Titan). The convective lapse rate is the starting point for theories of the depth of the troposphere (i.e., the height of the tropopause) in low latitudes (Held 1982; Thuburn and Craig 2000, Schneider 2007), which may have implications for the interpretation of observed radiance.

Latent heat release also affects the stratification of the extratropical atmosphere. The warm and moist sectors of extratropical cyclones condense as they move poleward and upward (Fig. 19). The vertical component of these transports is important in determining the lapse rate in the extratropics. Accounting for this interaction between extratropical cyclones and condensation or convection is an on-going area of research for Earth-like planets (Juckes 2000, Frierson et al. 2006; O'Gorman 2011).

In addition to the importance of the latent heat of condensation in determining the atmosphere's vertical thermal structure, it helps determine the horizontal temperature gradients. On Earth, about half of the meridional atmospheric

\footnotetext{
${ }^{16}$ Condensible species also affect the atmospheric mass, which is a minor effect on Earth, but is important on Mars and is potentially important on some exoplanets. See Section 4.4
} 
energy transport in midlatitudes is in the form of water vapor (e.g., Trenberth and Caron 2001). This is primarily accomplished by transient baroclinic eddies. The transient eddy moisture flux can be parameterized in terms of mean humidities and characteristic eddy velocities (Pierrehumbert 2002, O'Gorman and Schneider 2008; Caballero and Hanley 2012). In the low latitudes of Earth, the mean meridional circulation transports water vapor equatorward. This thermally indirect latent energy flux is a consequence of the small scale height of water vapor. The dry component of the energy flux is larger and poleward, so the total energy transport by the Hadley circulation is thermally direct. For Earth-like exoplanets, the energy transports by the day-side to night-side circulation will have a similar decomposition - a thermally indirect latent component and a thermally direct dry component (Merlis and Schneider 2010).

\subsubsection{Circulation energetics}

Latent heat release affects atmospheric circulations in a number of subtle ways. On the one hand, latent heat release has the potential to directly energize atmospheric circulations. On the other hand, the effect of latent heat release on the thermal structure of the atmosphere may weaken atmospheric circulations by increasing the static stability or decreasing horizontal temperature gradients.

The quantification of the kinetic energy cycle of the atmosphere is typically based on the dry thermodynamic budget, which is manipulated to form the Lorenz energy cycle (Lorenz 1955; Peixoto and Oort 1992). Latent heat release appears as an energy source in these budgets. For Earth's atmosphere, the diabatic generation of eddy kinetic energy from latent heat release is up to $\sim 1 \mathrm{~W} \mathrm{~m}^{-2}$, Chang 2001; Chang et al. 2002), which is a small fraction of the $\approx 80 \mathrm{~W} \mathrm{~m}^{-2}$ global-mean latent heat release (e.g., Trenberth et al. 2009). This low efficiency of converting latent heat into kinetic energy is consistent with the overall low efficiency of the kinetic energy cycle (Peixoto and Oort 1992). There are also alternative formulations of the kinetic energy cycle that incorporate moisture into the definition of the available potential energy (Lorenz 1978, 1979, O'Gorman 2010).

The energy transport requirements can play a role in determining the strength of the tropical circulations. For example, in angular momentum-conserving Hadley cell theories, the circulation energy transport is constrained to balance the top-of-atmosphere energy budget (Held and Hou 1980; Held 2000). Such an energy transport requirement does not directly determine the circulation mass transport, however. The ratio of the energy to mass transport is a variable known as the gross moist stability (other related definitions exist, Raymond et al. 2009). The gross moist stability expresses that the energy transport of tropical circulations results from the residual between the largely offsetting thermally indirect latent heat transport and the thermally direct dry static energy. Therefore, the gross moist stabil- ity is much smaller than the dry static stability for Earthlike atmospheres. The gross moist stability is an important, though uncertain, parameter in theories of tropical circulations (Neelin and Held 1987; Held 2000, 2001, Sobel 2007, Frierson 2007; Merlis et al.2012).

\section{ATMOSPHERIC CIRCULATION AND CLIMATE}

On any terrestrial planet, the influence of the atmospheric circulation on the climate is fundamental. On Earth, climate is generally understood as the statistics (long-term averages and variability) of surface pressure, temperature and wind, along with variations in the atmospheric content of water vapor and precipitation of liquid water or ice (Hartmann 1994b). For terrestrial bodies in general, this definition can readily be extended to include the atmospheric transport of other condensable species, as in the cases of the $\mathrm{CO}_{2}$ cycle on Mars or the methane cycle on Titan.

The close interaction between atmospheric dynamics and climate is readily apparent if we examine even the most basic features of the inner planets in our solar system. For example, Venus has a mean surface temperature of around $735 \mathrm{~K}$, with almost no variation between the equator and the poles. In contrast, Mars' mean surface temperature is only $210 \mathrm{~K}$, but in summer daytime temperatures can reach $290 \mathrm{~K}$, while in the polar winter conditions are cold enough for atmospheric $\mathrm{CO}_{2}$ to condense out as ice on the surface. Nonetheless, annual mean temperatures near the top of Olympus Mons are barely different from those in Mars' northern lowland plains. On Earth, surface temperature variations lie between these two extremes-the variation in climate with latitude is significant, but surface temperatures also rapidly decrease with altitude due to thermal coupling with the atmosphere. The differences in insolation, atmospheric pressure and composition (92 bar $\mathrm{CO}_{2}$ for Venus vs. around 1 bar $\mathrm{N}_{2} / \mathrm{O}_{2}$ for Earth and 0.006 bar $\mathrm{CO}_{2}$ for Mars) go a long way towards explaining these gross climatic differences. In particular, from planetary boundary layer theory it can be shown that the sensible heat exchange between the surface and atmosphere is

$$
\begin{aligned}
F_{\text {sens }} & =\overline{\rho_{a} w^{\prime}\left(c_{p} T^{\prime}\right)} \\
& \simeq c_{p} \rho_{a} C_{D}\left\|\mathbf{v}_{\text {surf }}\right\|\left(T_{\text {surf }}-T_{a}\right)
\end{aligned}
$$

where the overbar denotes a time or spatial mean, $w^{\prime}$ and $T^{\prime}$ are deviations from the mean vertical velocity and temperature, $T_{\text {surf }}$ is surface temperature, $C_{D}$ is a drag coefficient, $c_{p}$ is the specific atmospheric heat capacity, and $\rho_{a}$, $\left\|\mathbf{v}_{\text {surf }}\right\|$ and $T_{a}$ are the density, mean wind speed and temperature of the atmosphere near the surface (Pierrehumbert 2010). Because $F_{\text {sens }} \propto \rho_{a}$, the thermal coupling between the atmosphere and surface will generally increase with the atmospheric pressure. Given the tendency for dense, slowly rotating atmospheres to homogenize horizontal temperature differences rapidly (see Section 2.2), it should be intuitively clear why on planets like Venus, variations in annual mean temperature as a function of latitude and longitude tend to be extremely weak. 
In detail, the picture can be more complex, because $\left\|\mathbf{v}_{\text {surf }}\right\|$ is also a function of the atmospheric composition and insolation pattern, and $C_{D}$ depends on both the surface roughness and degree of boundary layer stratification. Beyond this, there are many other effects that couple with atmospheric dynamics. As discussed in Section 3, a key additional process on Earth is the hydrological cycle, which affects climate through latent heat transport and the radiative properties of water vapour and water ice/liquid clouds. Examples from other Solar System planets include the dust and $\mathrm{CO}_{2}$ cycles on Mars, and the methane/hydrocarbon haze cycle on Titan. These processes, which can be fascinatingly complex, are a large part of the reason why detailed long-term predictions of climate are challenging even for the relatively well-observed planets of the Solar System.

Given these complexities, it is unsurprising that for terrestrial exoplanets, study of the coupling between atmospheric circulation and climate in generalized cases is still in its infancy. The present state of knowledge is still heavily based on limited exploration of a few Earth-like or Earthsimilar cases. Rather than attempting an overview of the entire subject, therefore, here we review a small selection of the many situations where atmospheric dynamics is expected to have a key influence on planetary climate.

\subsection{Influence of dynamics on the runaway $\mathrm{H}_{2} \mathrm{O}$ green- house}

The well-known runaway $\mathrm{H}_{2} \mathrm{O}$ greenhouse effect occurs due to the feedback between surface temperature and atmospheric infrared opacity on a planet with surface liquid water. Above a given threshold for the incoming stellar flux $F_{0}>F_{\text {limit }}$ (see Fig 21, the thermal radiation leaving the planet no longer depends on the surface temperature, and water will continue to evaporate until all surface sources have disappeared (Kombayashi 1967, Ingersoll 1969). Classical runaway greenhouse calculations (e.g., Kasting et al. 1993) were performed in 1D and assumed homogenous atmospheres with either $100 \%$ relative humidity or a fixed vertical profile. In reality, however, the variations in relative humidity with latitude due to dynamical processes should have a fundamental effect on the planet's radiative budget and hence on the critical stellar flux at which the transition to a runaway greenhouse occurs. The global variations of relative humidity in Earth's atmosphere are strongly dependent on the ascending and descending motion of the Hadley cells (Section 2.2.1) and subtropical mixing due to synoptic-scale eddies (Section 2.1.2). Even on Earth today, without heat transport to higher latitudes by the atmosphere and ocean, the tropics would most likely be in a runaway greenhouse state ${ }^{17}$ Pierrehumbert (1995) proposed that regulation of mean tropical sea surface temperatures is in fact governed by a balance between the radiative heating in saturated upwelling regions and radiative cooling

\footnotetext{
${ }^{17}$ Assuming, that is, that the negative feedback from cloud albedo increases is not so strong as to overwhelm the increase in solar forcing past the conventional runaway greenhouse threshold.
}

by "radiator fins" in larger regions of net subsidence.

Very few researchers have yet taken on the challenge of modeling the transition of an ocean planet to a runaway greenhouse state in a 3D general circulation model (GCM). Ishiwatari et al. (2002) studied the appearance of the runaway greenhouse state in an idealized 3D circulation model with gray-gas radiative transfer. Their results roughly corresponded to those found in 1D using a global mean relative humidity of $60 \%$. However, the simplicity of their radiative scheme, neglect of clouds and problems arising from the need for strong vertical damping near the runaway state meant that they were unable to constrain the greenhouse transition for cases such as early Venus quantitatively. Later, Ishiwatari et al. (2007) compared results using a GCM with those from a 1D energy balance model (EBM). EBMs are intermediate-complexity models that replace vertically resolved radiative transfer with empirical functions for the fluxes at the top of the atmosphere and represent all latitudinal heat transport processes by a simple 1D diffusion equation. Ishiwatari et al. (2007) found that at intermediate values of the solar constant, multiple climate solutions including runaway greenhouse, globally and partially glaciated states were possible. Clearly, comparison of these results with simulations using a GCM specifically designed to remain physically robust in the runaway limit would be an interesting future exercise. As well as taking into account the non-gray radiative transfer of water vapour, such a model would need to account for the locally changing mass of water vapour in the large-scale dynamics and subgrid-scale convection, and include some representation of the radiative effects of clouds.

The importance of clouds to the runaway greenhouse limit is central, but our understanding of how they behave as the solar constant is increased is still poor. Kasting (1988) performed some simulations in 1D with fixed water cloud layers and concluded that because of their effect on the planetary albedo, Venus could easily have once been able to maintain surface liquid water. However, they did not include dynamical or microphysical cloud effects in their model. Going further, Rennó (1997) used a 1D model with a range of empirical parameterizations of cumulus convection to study the runaway transition, although with simplified cloud radiative transfer. Perhaps unsurprisingly, they found that surface temperature depends sensitively on cloud microphysical assumptions when solar forcing is increased. They also found that in general, mass flux schemes (i.e., convective parameterizations that capture the effect of environmental subsidence associated with cumulus convection) caused a more rapid runaway transition than adjustment schemes (i.e., convective parameterizations that simply represent the effect of convection as a modification of the local vertical temperature profile). Future 3D GCM studies of the runaway greenhouse will be forced to confront these uncertainties in sub-gridscale physical processes.

Cases where the initial planetary content of water is limited are also of interest for the runaway greenhouse. Abe et al. (2011) studied inner habitable zone limits for "land 

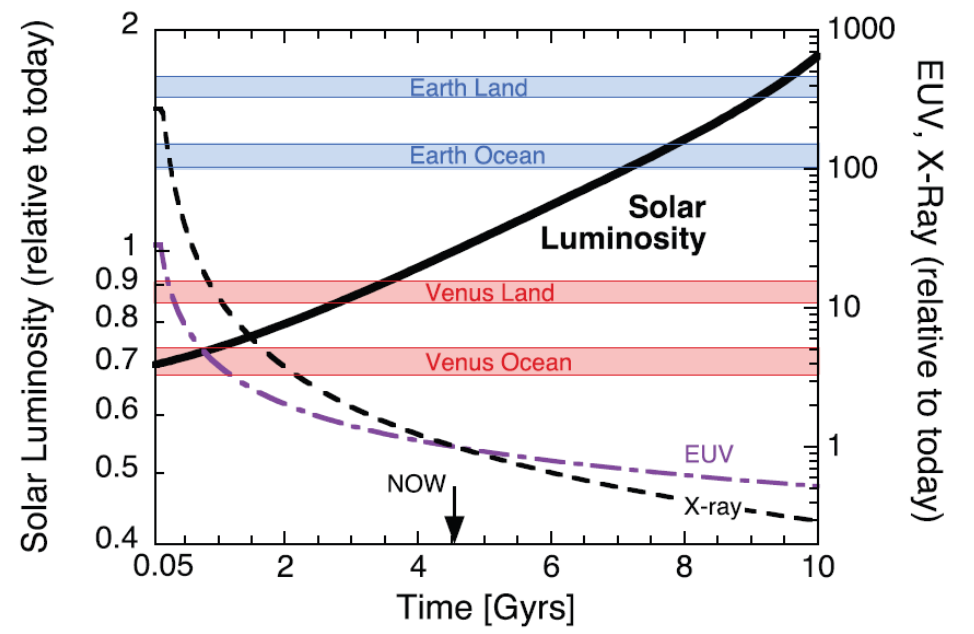

Fig. 21.- Solar luminosity as a function of time, alongside runaway $\mathrm{H}_{2} \mathrm{O}$ greenhouse thresholds for land and aqua planets at Venus and Earth's orbits (from Abe et al.2011). With a depleted water inventory, Venus could conceivably have remained a habitable planet until as little as 1 billion years ago.

planets", which they defined as planets with limited total $\mathrm{H}_{2} \mathrm{O}$ inventories (less than $1 \mathrm{~m}$ global average liquid equivalent in their model) and no oceans. They showed than in these cases, the tendency of the limited water inventory to become trapped at the poles causes extreme drying of equatorial regions. This allows the global mean outgoing longwave radiation to continue increasing with temperature, delaying the transition to a runaway greenhouse state. The critical average solar flux values they calculated in their model were $415 \mathrm{~W} \mathrm{~m}^{-2}$ and $330 \mathrm{~W} \mathrm{~m}^{-2}$ for land and aqua (global ocean) planets, respectively. Their results suggest planets with limited water inventories could remain habitable much closer to their host stars than the classical inner edge of the habitable zone suggests. Because the Sun's luminosity has increased with time, their results also suggest that a mainly dry early Venus could have stayed cool enough to maintain regions of surface liquid water until as recently as 1 billion years ago (see Figure 21). However, scenarios where liquid water was never present on the surface of Venus are also possible, and have been argued to be more consistent with Ne/Ar isotopic ratios and the low oxygen content in the present-day atmosphere (Gillmann et al. 2009, Chassefière et al. 2012).

The insights of Abe et al. (2011) on the importance of relative humidity to the runaway greenhouse have recently been extended to the case of tidally locked exoplanets around M-stars by Leconte et al. (2013). On a tidally locked terrestrial planet, the dark side may act as a cold trap for volatiles, which can lead to total collapse of the atmosphere in extreme cases, as we discuss later. For planets close to or inside the inner edge of the habitable zone, however, the effects of this process on $\mathrm{H}_{2} \mathrm{O}$ lead to an interesting bistability in the climate. One state consists of a classical runaway greenhouse where all the water is in the atmosphere. In the other state, the vast majority of the $\mathrm{H}_{2} \mathrm{O}$ is present as ice on the planet's dark side, while the planet's day side is relatively hot and extremely dry, allowing it to effectively radiate the incoming solar radiation back to space (see Figure 22). In this scenario, depending on the thickness of the ice sheet and the planet's thermal history, liquid water could be present in some amount near the ice sheet's edges, allowing marginal conditions for habitability well inside the classical inner edge of the habitable zone. Further work may be required to assess whether such a collapsed state would remain stable under the influence of transient melting events due to e.g., meteorite impacts on the planet's dark side.

\subsection{Snowball Earth dynamics and climate}

Runaway greenhouse states occur because of the effectiveness of gaseous $\mathrm{H}_{2} \mathrm{O}$ as an infrared absorber. However, the ability of water to cause fundamental changes in climate systems also extends to colder conditions. Solid $\mathrm{H}_{2} \mathrm{O}$ on the surface of a planet can have an equally drastic effect on circulation and climate, in this case due to its properties in the visible part of the spectrum.

Since the 1960s, it has been known that the Earth's climate has an alternative equilibrium state to present-day conditions. If the ice sheets that are currently confined to the poles instead extended all the way to the equator, the elevation in surface albedo would cause so much sunlight to be reflected back to space that mean global temperatures would drop to around $230-250 \mathrm{~K}$. As a result, surface and ocean ice would be stable even in the tropics, and the Earth could remain in a stable "snowball" state unless the greenhouse effect of the atmosphere increased dramatically. Snowball events are believed to have occurred at least twice previously in Earth's history: once around $640 \mathrm{Ma}$ and again $710 \mathrm{Ma}$, in the so-called Marinoan and Sturtian ice ages. Geological evidence (specifically, carbon isotope ratio excursions and observations of cap carbonates above 


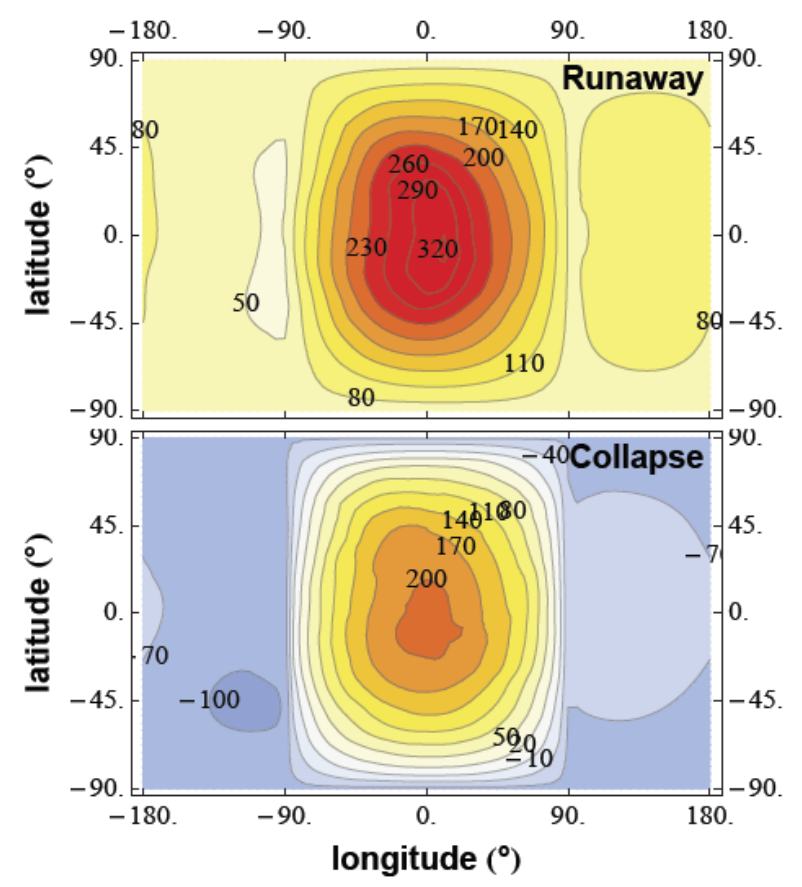

Fig. 22.- Surface temperature maps in ${ }^{\circ} \mathrm{C}$ from GCM simulations of a tidally locked, inner edge exoplanet with a 200mbar equivalent incondensible atmospheric component and fixed total water inventory. In the top panel, the planet was initialized with a mean water vapor column of $250 \mathrm{~kg} \mathrm{~m}^{-2}$ and remains in a runaway greenhouse state. In the bottom panel, the smaller water vapor starting inventory $\left(150 \mathrm{~kg} \mathrm{~m}^{-2}\right)$ has led to a transition to a collapsed state, with lower surface temperatures and ice on the planet's dark side.

glacial deposits) suggests that these events were ended by build-up of atmospheric $\mathrm{CO}_{2}$ to extremely high levels due to volcanic outgassing (Hoffman and Schrag 2002, Pierrehumbert et al. 2011). This scenario is particularly plausible because the land weathering of silicates and associated drawdown of $\mathrm{CO}_{2}$ into carbonates should be greatly reduced in a snowball climate (see Section 4.3.

Snowball Earth transitions are interesting in an exoplanetary context first because they represent a clear challenge to the concept of the habitability zone. If a habitable planet falls victim to snowball glaciation and cannot escape it, it may be irrelevant if it continues to receive the same flux from its host star. Identifying how these events can occur and how they end is hence of key importance in understanding the uniqueness of Earth's current climate. Snowball glaciation is also interesting from a purely physical viewpoint, as it is another example of a situation where the interplay between atmospheric dynamics and climate is fundamental to the problem. As we have seen in Section 2. heat transport between a planet's regions of high and low mean insolation is strongly dependent on the properties of the atmosphere and planetary rotation rate. As a result, the physics of the snowball transition should vary considerably between different exoplanet cases.

Recently, it has been hypothesized that changes in surface albedo due to atmospheric transport of dust may be critical to terminating Snowball Earth episodes (Abbot and
Pierrehumbert 2010 ). The reduced surface temperature and stellar energy absorption at the equator and absence of ocean heat transport on a globally frozen planet mean that typically, the tropics become a region of net ablation (i.e., mean evaporation exceeds precipitation). As a result, dust is slowly transported to the surface at the equator by a combination of horizontal glacial flow and vertical ice advection (see Figure 23. After a long time period, the buildup of dust on the surface lowers the albedo sufficiently to melt the surrounding ice and exit the planet from a snowball state. The fingerprints of the "mudball" state that presumably would follow this may have been imprinted on Earth's geological record, in the form of variations in thickness with (paleo)-latitude of clay drape deposits.

Other possibilities for snowball deglaciation come from water ice clouds. Pierrehumbert (2002) demonstrated using a one-dimensional radiative model that clouds have a net warming effect on a snowball planet. Because the surface albedo is high, clouds do not significantly increase the planetary albedo by reflecting incoming radiation to space. Nonetheless, they still effectively absorb outgoing IR radiation and hence cause greenhouse warming. These conclusions were broadly confirmed by a later 3D GCM intercomparison (Abbot et al.2012), although differences in cloud radiative forcing between models was found to be significant. Just as for the runaway greenhouse, future detailed studies of the physics of cloud formation and radiative 
transfer under snowball conditions, possibly using cloudresolving models, will be important for elucidating their role.

Further interesting couplings between atmospheric dynamics and climate arises if a planet near the snowball transition has a limited $\mathrm{H}_{2} \mathrm{O}$ inventory. Abe et al. (2011) studied idealized planets where the total amount of water (atmospheric and surface) only amounted to a $20-60 \mathrm{~cm}$ globally averaged layer. They showed that for these so-called "land" planets, the drying of tropical regions by a dynamical process similar to that responsible for dust accumulation would prevent the formation of stable surface ice there for a solar flux as low as $80 \%$ of that received by Earth today. As a result, the planetary albedo could remain low even if global mean surface temperatures dropped below the freezing point of $\mathrm{H}_{2} \mathrm{O}$, and transient regions of liquid water could persist longer than if the planet possessed as much water as Earth.

Finally, if the planet orbits a star of different spectral type to the Sun, further complications emerge. Recent work (Joshi and Haberle 2012) has demonstrated that around red dwarf M-stars, the mean albedos of snow and ice will be significantly reduced due to the red-shifted incident spectrum. As a result, the ice-albedo feedback is expected to be weaker, and snowball events may not be as difficult to exit as on Earth. Joshi and Haberle (2012) suggested that this effect may also help extend the outer edge of the habitable zone around M-stars. However, it is likely to be of lesser importance for planets with dense $\mathrm{CO}_{2}$ atmospheres, because the increased visible optical depth of the atmosphere and presence of $\mathrm{CO}_{2}$ clouds means the dependence of the planetary albedo on surface properties is already weak in these cases (Wordsworth et al.|2011).

\subsection{Carbonate-silicate weathering and atmospheric dynamics}

So far, we have focused on the coupling between circulation and climate in the context of $\mathrm{H}_{2} \mathrm{O}$. However, the role of other gases in climate may be equally significant. Carbon dioxide is of particular interest, given that it is the majority constituent of the atmospheres of both Venus and Mars. It is also the key greenhouse gas in our own planet's atmosphere, because of its special role in maintaining habitable surface conditions.

On Earth, the carbonate-silicate cycle is fundamental to the long-term evolution of carbon dioxide in the atmosphere. In particular, it is believed that the dependence of land silicate weathering on temperature leads to a negative feedback between atmospheric $\mathrm{CO}_{2}$ and mean surface temperature, rendering the climate stable to relatively large variations in solar luminosity (Walker et al. 1981). Indeed, the assumption of an efficient carbonate-silicate cycle lies behind the classical definition of the habitable zone (Kasting et al. 1993).

The idea that climate could naturally self-regulate on rocky planets due to an abiotic process is a highly attractive one, and probably a major part of the explanation for why Earth has maintained a (mostly) clement climate throughout its lifetime, despite significant increases in the solar flux (around 30\% since $4.4 \mathrm{Ga}$ ). Nonetheless, there are still major uncertainties in the nature of Earth's carbon cycle, including the importance of seafloor weathering (Sleep and Zahnle 2001; Le Hir et al. 2008), the exact role of plants in land weathering, and the dependence of the cycle on the details of plate tectonics.

Even given these uncertainties, it is already clear that substantial differences from the standard carbon cycle arise when a planet becomes synchronously locked. For example, Edson et al. (2012) investigated the relationship between weathering, atmospheric $\mathrm{CO}_{2}$ concentrations, and the distribution of continents for tidally locked planets around M-class stars. They coupled Earth GCM simulations with a simple parametrization for the global $\mathrm{CO}_{2}$ weathering rate, which following Walker et al. (1981) was written as

$$
\begin{aligned}
\frac{\left.d \mathrm{CO}_{2}\right|_{\text {tot }}}{d t}= & \frac{1}{2} \int W_{0}\left(\frac{f_{\mathrm{CO}_{2}}}{355 \mathrm{ppmv}}\right)^{0.3} \\
& \times\left(\frac{\mathcal{R}}{0.665 \mathrm{~mm} \mathrm{~d}^{-1}}\right) \exp \frac{T-T_{0}}{T_{U}} \mathrm{~d}(\mathbf{B} 3)
\end{aligned}
$$

where $f_{\mathrm{CO}_{2}}$ is the atmospheric $\mathrm{CO}_{2}$ volume mixing ratio, $W_{0}=8.4543 \times 10^{-10} \mathrm{C} \mathrm{s}^{-1} \mathrm{~m}^{-2}$ is the estimated presentday terrestrial weathering rate, $\mathcal{R}$ is the runoff rate, $T$ is temperature, $T_{0}=288 \mathrm{~K}, T_{U}=17.7 \mathrm{~K}$, and the integral is over the planet's weatherable (i.e., land) surface. By iterating between the simulations and Eqn. (33), Edson et al. (2012) derived self-consistent $f_{\mathrm{CO}_{2}}$ values as a function of the (constant) substellar longitude, given Earth's presentday geography. They found that $f_{\mathrm{CO}_{2}}$ could vary by a factor of $\sim 1 \times 10^{4}$ (7 ppmv vs. $60331 \mathrm{ppmv}$ ), depending on whether the substellar point was located over the Atlantic or Pacific oceans. As a result, the mean surface air temperatures in their simulations varied between 247 and $282 \mathrm{~K}$, with the climate in both cases similar to the "eyeball" state discussed in Pierrehumbert (2011) (see next section).

The differences in temperature and equilibrium atmospheric $\mathrm{CO}_{2}$ abundance between the two cases were caused by the differences in land area in the illuminated hemisphere. The illuminated hemisphere of a tidally locked planet is warmer, with higher precipitation rates (see Fig. 20), so a greater land area there implies enhanced global weathering rates and hence more rapid drawdown of $\mathrm{CO}_{2}$. Assuming that global $\mathrm{CO}_{2}$ levels are controlled by an expression like (33) is clearly an oversimplification given the complexity of Earth's real carbon cycle. In particular, basalt carbonization on the seafloor, which has a much weaker dependence on surface temperatures, is still poorly understood, but could have played a vital role in $\mathrm{CO}_{2}$ weathering throughout Earth's history. Nonetheless, the study highlights the dramatic differences that can be caused by 3D effects, and shows that a more detailed understanding of dynamical couplings with the carbonate-silicate cycle will 


\section{Tropical Dust Accumulation}

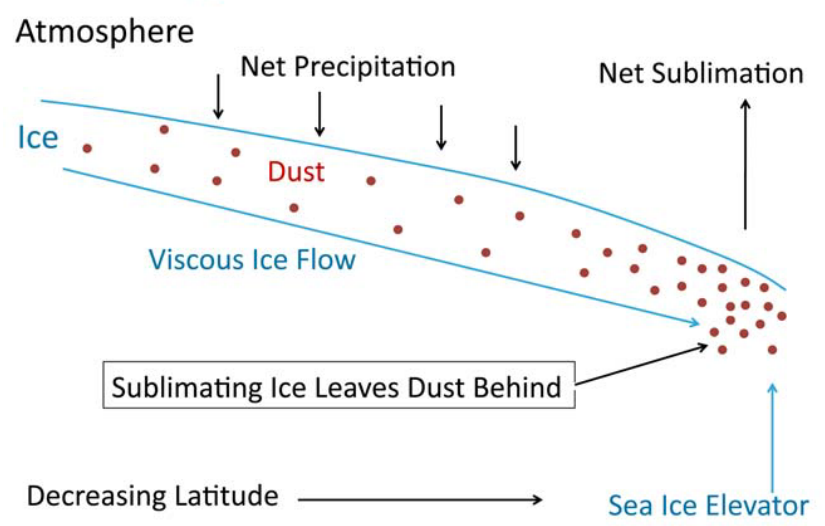

Fig. 23.- Schematic of the process by which tropical dust accumulates on a globally glaciated planet. The net sublimation at the tropics and precipitation in sub-tropical regions occurs primarily due to the reduced surface heat capacity of the surface when a thick ice layer is present (from Abbot and Pierrehumbert 2010).

be vital in future.

Kite et al. (2011) also explored the possible nature of climate-weathering feedbacks on tidally locked planets using an idealized energy balance climate model coupled to a simplified parametrization of silicate weathering. They noted that because heat transport efficiency depends on the atmospheric mass, in some cases reducing atmospheric pressure can increase the weathering rate and/or the liquid water volume on the day side of the planet. This can cause positive feedbacks that lead to a decrease in atmospheric mass. The mechanism described by Kite et al. (2011) is most likely to be important for planets like Mars, with thin, $\mathrm{CO}_{2}$-dominated atmospheres.

\subsection{Collapse of condensable atmospheres}

In Section 4.1, we briefly discussed situations where water vapour can become trapped as ice on the dark sides of tidally locked planets. Here, we discuss the extension of this process to the majority constituent of a planet's atmosphere. As we will see, atmospheric collapse is a problem of major importance to planetary habitability that sensitively depends on the details of the coupling between circulation and climate.

The interest in the habitability of tidally locked planets stems from the fact that cool, faint red dwarf stars (Mand $\mathrm{K}$ - spectral class) are significantly more common in the galaxy than stars like the Sun, and they host some of the lowest mass and best characterized exoplanets so far discovered (e.g., Udry et al. 2007; Mayor et al. 2009: Charbonneau et al. 2009; Bean et al. 2010). In the earliest detailed study of exoplanet habitability (Kasting et al. 1993), it was shown that planets around M-dwarf stars could sustain surface liquid water if they were in sufficiently close orbits. As discussed previously, the tidal interaction with their host stars means that such planets will in most cases have resonant or synchronous rotation rates and low obliquities, and hence permanent regions where little or no starlight reaches the surface. Initially, this was thought to be a potentially insurmountable obstacle to habitability, because the regions receiving no light could become so cold that any gas (including nitrogen) would freeze out on the surface there, depleting the atmosphere until the planet eventually became completely airless.

The problem of atmospheric collapse was first investigated quantitatively in a series of pioneering papers by Manoj Joshi and Bob Haberle (Joshi et al. 1997; Joshi 2003). Using a combination of basic scale analysis and 3D atmospheric modeling, they showed that the collapse of the atmosphere was only inevitable if it was inefficient at transporting heat. As we described earlier, the efficiency of atmospheric heat transport depends primarily on the composition (via the specific heat capacity and the infrared opacity), the average wind speed and (most critically) the total surface pressure. Assuming a surface emissivity of unity, the surface heat budget for a planet with condensible atmospheric species can be written

$$
\alpha \frac{\partial T_{\text {surf }}}{\partial t}=F_{s w}^{d n}+\left(F_{l w}^{d n}-\sigma T_{\text {surf }}^{4}\right)-L E-S H
$$

where $\sigma$ is the Stefan-Boltzmann constant, $\alpha$ is the surface heat capacity, $F_{s w}^{d n}$ and $F_{l w}^{d n}$ are the net downwelling fluxes of short- and longwave radiation from above, $L E$ is the latent heat flux, and $S H$ is the sensible heat flux, as described in (32). Equation (34) is essentially the unaveraged version of 29, except that for a single-component atmosphere, $L E$ only becomes important when the atmosphere begins to condense on the surface (as on present-day Mars, for example). On the dark side of a tidally locked planet $F_{s w}^{d n}=0$, so radiative cooling of the surface until the atmosphere begins to condense can be prevented in two ways: tight thermal coupling with the atmosphere via convection (the $S H$ term), or radiative heating of the surface by an optically thick atmosphere $\left(F_{l w}^{d n}\right)$. Both effects can 
be important in principle, although the temperature inversion caused by contact of warmer air from the dayside with the nightside surface may shut down convection, reducing the magnitude of the sensible heat flux or even reversing its sign. The strength of $F_{l w}^{d n}$ will depend on both the gas opacity of the atmosphere and the effects of clouds and aerosols, if present, and hence can be expected to vary with composition as well as atmospheric pressure. In any case, the calculations of Joshi et al. (1997) showed that for $\mathrm{CO}_{2}$ atmospheres with surface pressures of around $100 \mathrm{mBar}$ or more on Earth-like planets, both atmospheric transport and surface coupling become efficient enough to avoid the possibility of collapse and the climate can remain stable.

While Joshi and Haberle's simulations showed that synchronous rotation does not rule out the possibility of habitable climates around M-class stars, their work was focused on planets of Earth's mass and net stellar flux, and hence could not be applied to more general cases. Interest in this issue was reignited with the discovery of potentially habitable planets in the Gliese 581 system in 2007 (Udry et al. 2007; Mayor et al. 2009). Two planets, GJ581c and $\mathrm{d}$, orbit near the outer and inner edges of their system's habitable zone, and have estimated masses that suggest terrestrial rather than gas giant compositions. Simple one-dimensional models indicated that with a $\mathrm{CO}_{2}$-rich atmosphere, the "d" planet in particular could support surface liquid water (Wordsworth et al. 2010, von Paris et al. 2010; Hu and Ding 2011; Kaltenegger et al. 2011). Because M-stars have red-shifted spectra compared to the Sun, $\mathrm{CO}_{2}$ clouds typically cause less warming for planets around them, because the increase in planetary albedo they cause becomes nearly as significant as the greenhouse effect caused by their IR scattering properties. However, in $\mathrm{CO}_{2}$-rich atmospheres this is more than compensated for by increased near-IR absorption in the middle and lower atmosphere, which lowers the planetary albedo and hence increases warming.

The results from 1D models for GJ581d were suggestive, but the increase in condensation temperature with pressure means that atmospheric collapse becomes more of a threat as the amount of $\mathrm{CO}_{2}$ in the atmosphere increases. For example, reference to vapor pressure data for $\mathrm{CO}_{2}$ shows $p_{\text {sat }}=10$ bar at $\sim 235 \mathrm{~K}$, which is comparable to annual mean surface temperatures at Earth's south pole. It was therefore initially unclear if high- $\mathrm{CO}_{2}$ scenarios were plausible for GJ581d.

Wordsworth et al. (2011) investigated the stability of $\mathrm{CO}_{2}$-rich atmospheres on GJ581d using a general circulation model with band-resolved radiative transfer, simplified cloud physics, and evaporation/condensation cycles for $\mathrm{CO}_{2}$ and $\mathrm{H}_{2} \mathrm{O}$ included. They found that for this planet, which receives only around 30\% of Earth's insolation, the atmosphere could indeed be unstable to collapse even for pressures as high as 2-5 bar. Nonetheless, at higher pressures the simulated atmospheres stabilized due to a combination of the increased greenhouse warming and homogenization of the surface temperature by atmospheric heat transport. For atmospheric $\mathrm{CO}_{2}$ pressures of 10-30 bar (which are plausible given Venus and Earth's total $\mathrm{CO}_{2}$ inventories), Wordsworth et al. (2011) modeled surface temperatures for GJ581d in the $270-320 \mathrm{~K}$ range, with horizontal temperature variations of order 10-50 K (see Fig. 24). Sensitivity studies indicated that while the exact transition pressure from unstable to stable atmospheres was dependent on the assumed cloud microphysical properties, the general conclusion of stability at high $\mathrm{CO}_{2}$ pressure was not. The authors concluded that despite the low stellar flux it receives, GJ581d could therefore potentially support surface liquid water. As such, it is one of the most interesting currently known targets for follow-up characterization.

Any atmospheric observations of planets around Gliese 581 will be challenging, because the system does not appear to be aligned correctly for transit spectroscopy, and the planet-star contrast ratio constraints required for direct observations are severe. Distinguishing between habitable scenarios for GJ581d and other possibilities, such as a thin/collapsed atmosphere or a hydrogen-helium envelope, may be best accomplished via a search for absorption bands of $\mathrm{CO}_{2} / \mathrm{H}_{2} \mathrm{O}$ in the planet's emitted IR radiation that do not significantly vary over the course of one orbit (see Fig. 25. As described in Selsis et al. (2011), estimates of a nontransiting planet's atmospheric density are also possible, in principle, via analysis of the IR "variation spectrum" derived from phase curves. Despite the challenges, at 20 l.y. distance from the Sun, GJ581 is still a relatively close neighbor, and a future dedicated mission such as NASA's proposed Terrestrial Planet Finder (TPF) or ESA's Darwin would have the necessary capability to perform such observations.

Other recent studies have noted that even if global habitability is ruled out by the freezing of surface water on a planet's night side, it is still possible to maintain local regions of liquid water. Pierrehumbert (2011) investigated a series of possible climates for a $3.1 M_{\oplus}$ tidally locked exoplanet around an M-star receiving 63\% of Earth's incident solar flux. He hypothesized that if the planet's composition was dominated by $\mathrm{H}_{2} \mathrm{O}$, it would most likely have an icy surface and hence a high albedo. However, if the atmosphere were extremely thin and hence inefficient at transporting heat, surface temperatures near the substellar point could allow some $\mathrm{H}_{2} \mathrm{O}$ to melt, even though the global mean temperature would be around $192 \mathrm{~K}$ if heat transport were efficient. Given a denser mixed $\mathrm{N}_{2}-\mathrm{CO}_{2}-\mathrm{H}_{2} \mathrm{O}$ atmosphere, an "eyeball" climate could also be maintained where permanent ice is present on the night side, but temperatures are high enough on the day side to allow an ocean to form (see Fig. 26). Although such a scenario is admittedly hypothetical, it raises many interesting questions on the general nature of exoplanet climate and may help with the interpretation of observations in the future.

Clearly, the likelihood of atmospheric collapse also depends strongly on the condensation temperature of the majority gas in the atmosphere. Despite this progress in modeling exotic exoplanet atmospheres in specific cases, the gen- 

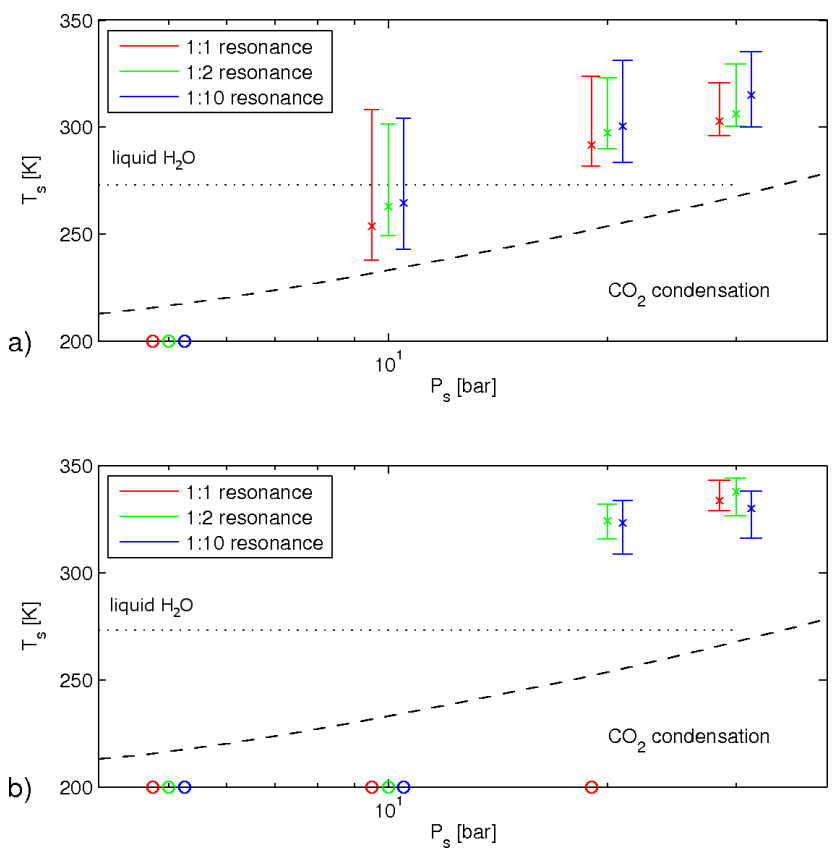

Fig. 24.- Simulated annual mean surface temperature (maximum, minimum and global average) as a function of atmospheric pressure and rotation rate for GJ581d assuming a) a pure $\mathrm{CO}_{2}$ atmosphere and b) a mixed $\mathrm{CO}_{2}-\mathrm{H}_{2} \mathrm{O}$ atmosphere with infinite water source at the surface [from Wordsworth et al. (2011)]. Data plotted with circles indicate where the atmosphere had begun to collapse on the surface in the simulations, and hence no steady-state temperature could be recorded. In the legend, 1:1 resonance refers to a synchronous rotation state, and 1:2 and 1:10 resonances refer to despun but asynchronous spin-orbit configurations.

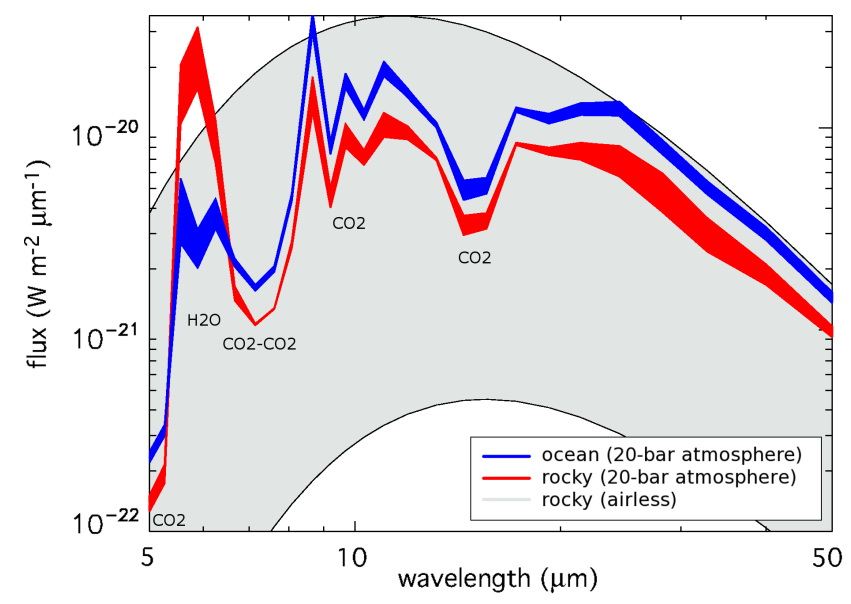

Fig. 25.- Simulated emission spectra for GJ581d given various atmospheric scenarios [from Wordsworth et al. (2011)]. In all cases the thicknesses of the lines correspond to the range of variation predicted over the course of one planetary orbit. The relatively low variation in the red/blue cases is a characteristic signature of a planet with a stable, dense atmosphere with efficient horizontal heat redistribution. 


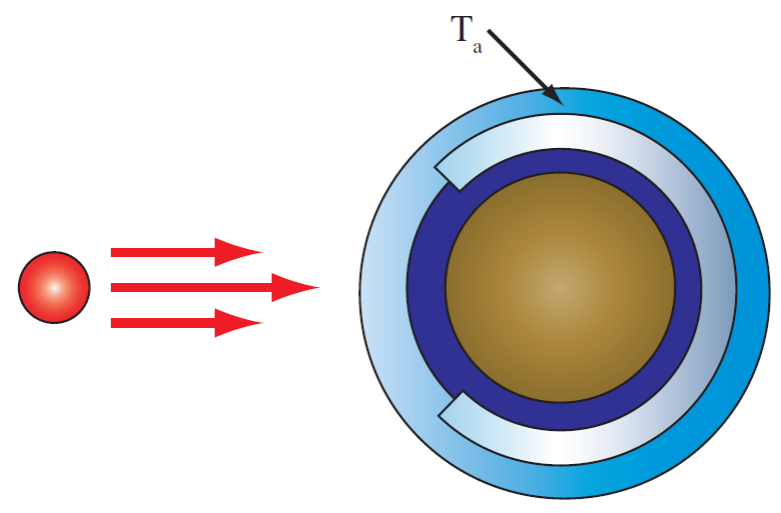

Fig. 26.- Schematic of the "eyeball" climate state for synchronously rotating terrestrial planet (Pierrehumbert|2011). Sea ice is present across the permanent night side of the planet, but stops at a stellar zenith angle primarily determined by the balance between the local stellar flux and horizontal atmospheric and oceanic heat transport.

eral problem of atmospheric condensation in a planet's cold trap(s) for arbitrary conditions remains unsolved. Nonetheless, scale analysis has recently been used to place some basic general constraints on atmospheric stability by Heng and Kopparla (2012). These authors assumed the criterion $\tau_{\text {rad }}>\tau_{\text {adv }}$ as a condition for stability, where $\tau_{\text {rad }}$ and $\tau_{\text {adv }}$ are representative timescales for radiative cooling and advection, respectively. The radiative timescale can be approximately defined as $\tau_{\text {rad }}=c_{p} p / g \sigma T_{\text {rad }}^{3}$, with $p$ the total pressure, $g$ gravity and $T_{\text {rad }}$ the characteristic emission temperature, while $\tau_{\mathrm{adv}} \sim L / U$, with $L$ and $U$ a characteristic horizontal length and wind speed, respectively. Evaluating $\tau_{\text {rad }}$ is straightforward for a known atmospheric composition, but $U$ and hence $\tau_{\text {adv }}$ are difficult to assess a priori without a general theory for the response of the atmospheric circulation to different forcing scenarios. Heng and Kopparla (2012) neatly circumvented this problem by simply taking $U$ to be the speed of sound, allowing a potentially strict criterion for atmospheric collapse based entirely on known properties of the atmosphere.

Despite its elegance, their approach was limited by its neglect of the role of thermodynamics in the problem. In particular, a moving gas parcel with a majority constituent that condenses at low temperatures may continue to radiate until the local value of $\tau_{\text {rad }}$ becomes large, which tends to make an atmosphere much more resistant to collapse. There is still considerable scope for theoretical development of the atmospheric collapse problem, and given its fundamental importance to the understanding of both atmospheric circulation and habitability around M-stars, it is likely that it will continue to receive attention for some time.

The dependence of collapse on saturation vapour temperature is particularly interesting when considered in the context of more exotic scenarios for terrestrial planet atmospheric composition. In particular, the saturation vapour temperatures of hydrogen and helium at 1 bar are under $25 \mathrm{~K}$, rendering them essentially incondensible on planets receiving even very little energy from their host stars.
Many rocky planets are believed to form with thin primordial envelopes of these gases, which have large optical depths in the infrared for pressures above a fraction of a bar. For planets of Earth's mass or lower, these envelopes generally escape rapidly under the elevated stellar wind and extreme ultraviolet (XUV) fluxes from young stars. However, higher mass or more distant planets may experience a range of situations where hydrogen and helium can remain in their atmospheres for longer periods. Stevenson (1999) suggested that some planetary embryos ejected from their system during formation could sustain liquid oceans if they kept their primordial hydrogen envelopes. Pierrehumbert and Gaidos (2011) extended this idea to planets in distant orbits from their stars. These planets could be detectable in theory due to gravitational microlensing, although given their low equilibrium temperatures, atmospheric characterization would be extremely challenging even with the most ambitious planned missions. Wordsworth (2012) noted that in general, very few planets are left with just the right amount of hydrogen in their atmospheres to maintain surface liquid water after the initial period of intense XUV fluxes and rapid hydrogen escape. Nonetheless, they showed that transient periods of surface habitability (between $\sim 10,000 \mathrm{yr}$ and $\sim 100 \mathrm{Myr}$ ) still occur for essentially any planets receiving less flux than Earth that form with a hydrogen envelope but later lose it. In addition, XUV photolysis in hydrogen-rich atmospheres can lead to the formation of a range of pre-biotic compounds (Miller 1953, DeWitt et al. 2009). Conditions on young terrestrial planets that at least allow life to form could hence be much more common than previously assumed.

\section{CONCLUSIONS}

Given the diversity emerging among more massive exoplanets, it is almost certain that terrestrial exoplanets will occupy an incredible range of orbital and physical parameters, including orbital semi-major axis, orbital eccentricity, incident stellar flux, incident stellar spectrum, plane- 
tary rotation rate, obliquity, atmospheric mass, composition, volatile inventory (including existence and mass of oceans), and evolutionary history. Since all of these parameters affect the atmospheric dynamics, it is likewise probable that terrestrial exoplanets will exhibit incredible diversity in the specific details of their atmospheric circulation patterns and climate. Only a small fraction of this diversity has yet been explored in GCMs and similar models. As we have emphasized in this review, existing theory suggests that a number of unifying themes will emerge governing the atmospheric circulation on this broad class of bodies. This theory-summarized here-will provide a foundation for understanding, and provide a broad context for, the results of GCM investigations of particular objects.

Exoplanets that rotate sufficiently rapidly will exhibit extratropics at high latitudes, where the dynamics are approximately geostrophic, and where eddies resulting from baroclinic instabilities control the meridional heat transport, equator-to-pole temperature differences, meridional mixing rates, existence of jet streams, and thermal stratification in the troposphere. Regions where rotation is less dominant-near the equator, and globally on slowly rotating exoplanets-will exhibit tropical regimes where wave adjustment and Hadley circulations typically act to minimize horizontal temperature differences. Significant interactions between the tropics and extratropics can occur by a variety of mechanisms, perhaps the most important of which is the propagation of Rossby waves between the two regions. The hydrological cycle on many terrestrial exoplanets will exert significant effects both on the mean climate-through the greenhouse effect and clouds-and on the circulation patterns. Existing studies demonstrate that the circulation can influence global-scale climate feedbacks, including the runaway greenhouse, transitions to snowball-Earth states, and atmospheric collapse, leading to a partial control of atmospheric circulation on the mean climate and therefore planetary habitability.

Exoplanets can exhibit greatly different regimes of thermal forcing than occur on solar system planets, a topic which has yet to be fully explored. The day-night thermal forcing on sychronously rotating and despun exoplanets will play a more important role than on the Earth, leading in many cases to equatorial superrotation and other dynamical effects. When the heating rates are sufficiently great (in the limit of thin and/or hot atmospheres), the heating and cooling gradients can overwhelm the ability of the atmosphere to transport heat horizontally, leading to freeze out of the more refractory components (such as water or carbon dioxide) on the nightside or poles. This regime remains poorly understood, although it is important for understanding the structure, and even existence, of atmospheres on hot terrestrial planets and super Earths.

Observations will be needed to move the field forward. These in many cases will represent extensions to terrestrial planets of techniques currently being applied to hot Jupiters. Short-term, gains are most likely for transiting planets, particularly those around small $\mathrm{M}$ stars: a terrestrial planet or- biting such a star exhibits a planet/star radius ratio similar to that of a gas giant orbiting a Sun-like star, thereby making characterization (relatively) easier. Transit spectroscopy of such systems will yield constraints on atmospheric gas composition and existence of hazes (e.g., Barman 2007, Sing et al. 2008; Pont et al. 2012, Bean et al. 2010, Désert et al. 2011; Berta et al. 2012). In the longer-term future, such spectroscopy could also provide direct measurements of atmospheric wind speeds at the terminator (Snellen et al. 2010, Hedelt et al. 2011). Secondary eclipse detections and, eventually, full-orbit lightcurves will provide information on the emission spectrum as a function of longitude, allowing constraints on the vertical temperature profile and daynight temperature difference to be inferred. This will first be possible for hot systems (Demory et al.2012), but with significant investment of resources from the James Webb Space Telescope will also be possible for planets in the habitable zones (Seager et al. 2008). Eclipse mapping may also eventually be possible, as is now being performed for hot Jupiters (Majeau et al. 2012, de Wit et al. 2012). Using direct imaging to obtain spectroscopy of planets is another observational avenue - not limited to transiting systemsthat could be performed from a platform like the Terrestrial Planet Finder. All of these observations will be a challenge, but the payoff will be significant: the first characterization of terrestrial worlds around stars in the solar neighborhood.

In the meantime, theory and models can help to address open questions concerning the behavior of atmospheric circulation and climate across a wider range of parameter space than encountered in the solar system. Major questions emphasized in this review include the following: What is the dependence of three-dimensional temperature structure, humidity, and horizontal heat flux on planetary rotation rate, incident stellar flux, atmospheric mass, atmospheric composition, planetary radius and gravity, and other conditions? What are the regimes of atmospheric wind? What is the influence of an ocean in modulating the atmospheric climate? Under what conditions can clouds form, and what is their three-dimensional distribution for planets of various types? How do seasonal cycles (due to non-zero obliquity and/or orbital eccentricity) affect the atmospheric circulation and climate on planets generally? What is the role of the atmospheric circulation - through its control of temperature, humidity, cloudiness, and precipitation - in affecting longer-term climatic processes including runaway greenhouses, ice-age cycles, transitions to snowball-Earth states, the carbonate-silicate feedback, and collapse of condensable atmospheric constituents onto the poles or nightside? And what is the continuum of atmospheric behaviors on exoplanets ranging from sub-Earth-sized terrestrial planets to super Earths and mini-Neptunes? While a detailed understanding of particular exoplanets must await observations, significant insights into the fundamental physical mechanisms controlling atmospheric circulation and climateof planets generally - can be made now with current theoretical and modeling tools. This will not only lay the groundwork for understanding future observations but will 
place the atmospheric dynamics and climate of solar-system worlds, including Earth, into its proper planetary context.

Acknowledgments. We thank Jonathan Mitchell and an anonymous referee for thorough reviews of the manuscript. This paper was supported by NASA grants NNX10AB91G and NNX12AI79G to APS. TMM was supported by a Princeton Center for Theoretical Science fellowship.

\section{REFERENCES}

Abbot, D. S., and R. T. Pierrehumbert, 2010: Mudball: Surface dust and Snowball Earth deglaciation. Journal of Geophysical Research (Atmospheres), 115(D14), 3104.

Abbot, D. S., A. Voigt, M. Branson, R. T. Pierrehumbert, D. Pollard, G. Le Hir, and D. D. B. Koll, 2012: Clouds and Snowball Earth deglaciation. Geophys. Res. Lett., 39, 20,711.

Abe, Y., A. Abe-Ouchi, N. H. Sleep, and K. J. Zahnle, 2011: Habitable Zone Limits for Dry Planets. Astrobiology, 11, 443-460.

Adam, O., and N. Paldor, 2009: Global Circulation in an Axially Symmetric Shallow Water Model Forced by Equinoctial Differential Heating. Journal of Atmospheric Sciences, 66, 1418.

Adam, O., and N. Paldor, 2010: Global Circulation in an Axially Symmetric Shallow-Water Model, Forced by Off-Equatorial Differential Heating. Journal of Atmospheric Sciences, 67, 1275-1286.

Allen, M. R., and W. J. Ingram, 2002: Constraints on future changes in climate and the hydrologic cycle. Nature, 419, 224232.

Andrews, D. G., J. R. Holton, and C. B. Leovy, 1987: Middle Atmosphere Dynamics. Academic Press, New York.

Arnold, N. P., E. Tziperman, and B. Farrell, 2012: Abrupt Transition to Strong Superrotation Driven by Equatorial Wave Resonance in an Idealized GCM. Journal of Atmospheric Sciences, 69, 626-640.

Back, L. E., and C. S. Bretherton, 2009a: On the relationship between SST gradients, boundary layer winds, and convergence over the tropical oceans. J. Climate, 22, 4182-4196.

Back, L. E., and C. S. Bretherton, 2009b: A simple model of climatological rainfall and vertical motion patterns over the tropical oceans. J. Climate, 22, 6477-6497.

Bala, G., P. B. Duffy, and K. E. Taylor, 2008: Impact of geoengineering schemes on the global hydrological cycle. Proc. Nat. Acad. Sci., 105, 7664-7669.

Barman, T., 2007: Identification of Absorption Features in an Extrasolar Planet Atmosphere. Astrophys. J. Lett., 661, L191L194.

Barry, L., G. C. Craig, and J. Thuburn, 2002: Poleward heat transport by the atmospheric heat engine. Nature, 415, 774-777.

Bean, J. L., E. Kempton, and D. Homeier, 2010: A ground-based transmission spectrum of the super-Earth exoplanet GJ 1214b. Nature, 468, 669-672.

Beron-Vera, F. J., M. G. Brown, M. J. Olascoaga, I. I. Rypina, H. Koçak, and I. A. Udovydchenkov, 2008: Zonal Jets as Transport Barriers in Planetary Atmospheres. Journal of Atmospheric Sciences, 65, 3316.
Berta, Z. K., et al., 2012: The Flat Transmission Spectrum of the Super-Earth GJ1214b from Wide Field Camera 3 on the Hubble Space Telescope. Astrophys. J., 747, 35.

Betts, A. K., 1974: Further comments on comparison of the equivalent potential temperature and the static energy. J. Atmos. Sci., 31, 1713-1715.

Bony, S., et al., 2006: How well do we understand and evaluate climate change feedback processes? J. Climate, 19, 34453482.

Bordoni, S., and T. Schneider, 2008: Monsoons as eddy-mediated regime transitions of the tropical overturning circulation. $\mathrm{Na}$ ture Geoscience, 1, 515-519.

Bordoni, S., and T. Schneider, 2010: Regime Transitions of Steady and Time-Dependent Hadley Circulations: Comparison of Axisymmetric and Eddy-Permitting Simulations. Journal of Atmospheric Sciences, 67, 1643-1654.

Borucki, W. J., et al., 2011: Characteristics of Planetary Candidates Observed by Kepler. II. Analysis of the First Four Months of Data. Astrophys. J., 736, 19.

Borucki, W. J., et al., 2012: Kepler-22b: A 2.4 Earth-radius Planet in the Habitable Zone of a Sun-like Star. Astrophys. J., 745, 120.

Borucki, W. J., et al., 2013: Kepler-62: A five-planet system with planets of 1.4 and 1.6 Earth radii in the habitable zone. Science (Sciencexpress).

Bretherton, C. S., and P. K. Smolarkiewicz, 1989: Gravity Waves, Compensating Subsidence and Detrainment around Cumulus Clouds. Journal of Atmospheric Sciences, 46, 740-759.

Caballero, R., and J. Hanley, 2012: Midlatitude eddies, stormtrack diffusivity and poleward moisture transport in warm climates. J. Atmos. Sci., p. in press.

Caballero, R., and M. H. Huber, 2010: Spontaneous transition to superrotation in warm climates simulated by CAM3. Geophys. Res. Lett., p. in press.

Caballero, R., R. T. Pierrehumbert, and J. L. Mitchell, 2008: Axisymmetric, nearly inviscid circulations in non-condensing radiative-convective atmospheres. Quarterly Journal of the Royal Meteorological Society, 134, 1269-1285.

Castan, T., and K. Menou, 2011: Atmospheres of Hot SuperEarths. Astrophys. J. Lett., 743, L36.

Chang, E. K. M., 2001: GCM and observational diagnoses of the seasonal and interannual variations of the Pacific storm track during the cool season. J. Atmos. Sci., 58, 1784-1800.

Chang, E. K. M., S. Lee, and K. L. Swanson, 2002: Storm track dynamics. J. Climate, 15, 2163-2183.

Charbonneau, D., et al., 2009: A super-Earth transiting a nearby low-mass star. Nature, 462, 891-894.

Charney, J. G., 1963: A Note on Large-Scale Motions in the Tropics. Journal of Atmospheric Sciences, 20, 607-608.

Chassefière, E., R. Wieler, B. Marty, and F. Leblanc, 2012: The evolution of Venus: Present state of knowledge and future exploration. Planetary and Space Science, 63, 15-23.

Cho, J. Y.-K., and L. M. Polvani, 1996: The morphogenesis of bands and zonal winds in the atmospheres on the giant outer planets. Science, 8(1), 1-12. 
Chou, C., and J. D. Neelin, 2004: Mechanisms of global warming impacts on regional tropical precipitation. J. Climate, 17, 2688-2701.

Cooper, C. S., and A. P. Showman, 2005: Dynamic Meteorology at the Photosphere of HD 209458b. Astrophys. J. Lett., 629, L45-L48.

Couhert, A., T. Schneider, J. Li, D. E. Waliser, and A. M. Tompkins, 2010: The maintenance of the relative humidity of the subtropical free troposphere. J. Climate, 23, 390-403.

Cowan, N. B., and E. Agol, 2011: The Statistics of Albedo and Heat Recirculation on Hot Exoplanets. Astrophys. J., 729, 54.

Cowan, N. B., et al., 2009: Alien Maps of an Ocean-bearing World. Astrophys. J., 700, 915-923.

de Wit, J., M. Gillon, B.-O. Demory, and S. Seager, 2012: Towards consistent mapping of distant worlds: secondary-eclipse scanning of the exoplanet HD 189733b. Astron. Astrophys., 548, A128.

Del Genio, A. D., and R. J. Suozzo, 1987: A comparative study of rapidly and slowly rotating dynamical regimes in a terrestrial general circulation model. Journal of Atmospheric Sciences, 44, 973-986.

Del Genio, A. D., and W. Zhou, 1996: Simulations of Superrotation on Slowly Rotating Planets: Sensitivity to Rotation and Initial Condition. Icarus, 120, 332-343.

Del Genio, A. D., W. Zhou, and T. P. Eichler, 1993: Equatorial superrotation in a slowly rotating GCM - Implications for Titan and Venus. Icarus, 101, 1-17.

Del Genio, A. D., R. K. Achterberg, K. H. Baines, F. M. Flasar, P. L. Read, A. Sánchez-Lavega, and A. P. Showman, 2009: Saturn Atmospheric Structure and Dynamics. Saturn from Cassini-Huygens (Dougherty, M. K., Esposito, L. W. and Krimigis, S. M., Eds.), Springer, p. 113.

Deming, D., and S. Seager, 2009: Light and shadow from distant worlds. Nature, 462, 301-306.

Demory, B.-O., M. Gillon, S. Seager, B. Benneke, D. Deming, and B. Jackson, 2012: Detection of Thermal Emission from a Super-Earth. Astrophys. J. Lett., 751, L28.

Désert, J.-M., J. Bean, E. Miller-Ricci Kempton, Z. K. Berta, D. Charbonneau, J. Irwin, J. Fortney, C. J. Burke, and P. Nutzman, 2011: Observational Evidence for a Metal-rich Atmosphere on the Super-Earth GJ1214b. Astrophys. J. Lett., 731, L40.

DeWitt, H. L., M. G. Trainer, A. A. Pavlov, C. A. Hasenkopf, A. C. Aiken, J. L. Jimenez, C. P. McKay, O. B. Toon, and M. A. Tolbert, 2009: Reduction in Haze Formation Rate on Prebiotic Earth in the Presence of Hydrogen. Astrobiology, 9, 447-453.

Dritschel, D. G., and M. E. McIntyre, 2008: Multiple Jets as PV Staircases: The Phillips Effect and the Resilience of EddyTransport Barriers. Journal of Atmospheric Sciences, 65, 855.

Dritschel, D. G., and R. K. Scott, 2011: Jet sharpening by turbulent mixing. Phil. Trans. Roy. Soc. A, 369, 754-770.

Dunkerton, T. J., and R. K. Scott, 2008: A Barotropic Model of the Angular Momentum Conserving Potential Vorticity Staircase in Spherical Geometry. Journal of Atmospheric Sciences, 65 , 1105.
Edson, A., S. Lee, P. Bannon, J. F. Kasting, and D. Pollard, 2011: Atmospheric circulations of terrestrial planets orbiting lowmass stars. Icarus, 212, 1-13.

Edson, A. R., J. F. Kasting, D. Pollard, S. Lee, and P. R. Bannon, 2012: The Carbonate-Silicate Cycle and CO2/Climate Feedbacks on Tidally Locked Terrestrial Planets. Astrobiology, 12, 562-571.

Emanuel, K. A., 1994: Atmospheric Convection. Oxford University Press.

Enderton, D., and J. Marshall, 2009: Explorations of atmosphereocean-ice climates on an aquaplanet and their meridional energy transports. J. Atmos. Sci., 66, 1593-1611.

Fang, M., and K. K. Tung, 1996: A simple study of nonlinear Hadley circulation with an ITCZ: analytic and numerical solutions. J. Atmos. Sci., 53, 1241-1261.

Farrell, B. F., 1990: Equable Climate Dynamics. Journal of Atmospheric Sciences, 47, 2986-2995.

Ferrari, R., and D. Ferreira, 2011: What processes drive the ocean heat transport? Ocean Modelling, 38, 171-186.

Flasar, F. M., K. H. Baines, M. K. Bird, T. Tokano, and R. A. West, 2009: Atmospheric dynamics and meteorology. Titan from Cassini-Huygens (Brown, R.H., Lebreton, J-P., and Waite, J.H., Eds.), Springer, pp. 323-352.

Fressin, F., et al., 2012: Two Earth-sized planets orbiting Kepler20. Nature, 482, 195-198.

Frierson, D. M. W., 2007: The dynamics of idealized convection schemes and their effect on the zonally averaged tropical circulation. J. Atmos. Sci., 64, 1959-1976.

Frierson, D. M. W., I. M. Held, and P. Zurita-Gotor, 2006: A GrayRadiation Aquaplanet Moist GCM. Part I: Static Stability and Eddy Scale. Journal of Atmospheric Sciences, 63, 2548-2566.

Fujii, Y., and H. Kawahara, 2012: Mapping Earth Analogs from Photometric Variability: Spin-Orbit Tomography for Planets in Inclined Orbits. Astrophys. J., 755, 101.

Fujii, Y., H. Kawahara, Y. Suto, S. Fukuda, T. Nakajima, T. A. Livengood, and E. L. Turner, 2011: Colors of a Second Earth. II. Effects of Clouds on Photometric Characterization of Earthlike Exoplanets. Astrophys. J., 738, 184.

Galewsky, J., A. Sobel, and I. Held, 2005: Diagnosis of subtropical humidity dynamics using tracers of last saturation. J. Atmos. Sci., 62, 3353-3367.

Garcia, R. R., and M. L. Salby, 1987: Transient Response to Localized Episodic Heating in the Tropics. Part II: Far-Field Behavior. Journal of Atmospheric Sciences, 44, 499-532.

Garratt, J. R., 1994: The Atmospheric Boundary Layer. Cambridge University Press.

Gierasch, P. J., et al., 1997: The General Circulation of the Venus Atmosphere: an Assessment. Venus II: Geology, Geophysics, Atmosphere, and Solar Wind Environment, S. W. Bougher, D. M. Hunten, and R. J. Philips, Eds., pp. 459-500.

Gill, A. E., 1980: Some simple solutions for heat-induced tropical circulation. Q. J. Roy. Meteor. Soc., 106, 447-462.

Gillmann, C., E. Chassefière, and P. Lognonné, 2009: A consistent picture of early hydrodynamic escape of Venus atmosphere explaining present $\mathrm{Ne}$ and $\mathrm{Ar}$ isotopic ratios and low oxygen atmospheric content. Earth and Planetary Science Letters, 286, 503-513. 
Gómez-Leal, I., E. Pallé, and F. Selsis, 2012: Photometric Variability of the Disk-integrated Thermal Emission of the Earth. Astrophys. J., 752, 28.

Green, J. S. A., 1970: Transfer properties of the large-scale eddies and the general circulation of the atmosphere. Quarterly Journal of the Royal Meteorological Society, 96, 157-185.

Haine, T. W. N., and J. Marshall, 1998: Gravitational, Symmetric, and Baroclinic Instability of the Ocean Mixed Layer. Journal of Physical Oceanography, 28, 634-658.

Hartmann, D. L., 1994a: A PV view of zonal mean flow vacillation, submitted to J. Atmos. Sci.

Hartmann, D. L., 1994b: Global physical climatology. vol. 56, Academic press.

Hayashi, Y.-Y., K. Ishioka, M. Yamada, and S. Yoden, 2000: Emergence of circumpolar vortex in two dimensional turbulence on a rotating sphere. Proceedings of the IUTAM Symposium on Developments in Geophysical Turbulence (Fluid Mechanics and its Applications V. 58, R. M. Kerr and Y. Kimura, Eds., Kluwer Academic Pub., pp. 179-192.

Hayashi, Y.-Y., S. Nishizawa, S.-I. Takehiro, M. Yamada, K. Ishioka, and S. Yoden, 2007: Rossby Waves and Jets in TwoDimensional Decaying Turbulence on a Rotating Sphere. Journal of Atmospheric Sciences, 64, 4246-4269.

Hedelt, P., R. Alonso, T. Brown, M. Collados Vera, H. Rauer, H. Schleicher, W. Schmidt, F. Schreier, and R. Titz, 2011: Venus transit 2004: Illustrating the capability of exoplanet transmission spectroscopy. Astron. Astrophys., 533, A136.

Held, I. M., 1975: Momentum transport by quasi-geostrophic eddies. Journal of the Atmospheric Sciences, 32, 1494-1497.

Held, I. M., 1982: On the height of the tropopause and the static stability of the troposphere. J. Atmos. Sci., 39, 412-417.

Held, I. M., 1999a: The macroturbulence of the troposphere. Tellus, 51A-B, 59-70.

Held, I. M., 1999b: Equatorial superrotation in Earthlike atmospheric models. Bernhard Haurwitz Memorial Lecture, American Meteorological Society, available at www.gfdl.noaa.gov/isaac-held-homepage.

Held, I. M., 2000: The general circulation of the atmosphere. Paper presented at 2000 Woods Hole Oceanographic Institute Geophysical Fluid Dynamics Program, Woods Hole Oceanographic Institute, Woods Hole, MA (available at http://www.whoi.edu/page.do?pid=13076).

Held, I. M., 2001: The partitioning of the poleward energy transport between the tropical ocean and atmosphere. J. Atmos. Sci., 58, 943-948.

Held, I. M., and A. Y. Hou, 1980: Nonlinear Axially Symmetric Circulations in a Nearly Inviscid Atmosphere. Journal of Atmospheric Sciences, 37, 515-533.

Held, I. M., and V. D. Larichev, 1996: A scaling theory for horizontally homogeneous, baroclinically unstable flow on a beta plane. Journal of Atmospheric Sciences, 53, 946-952.

Held, I. M., and K. M. Shell, 2012: Using relative humidity as a state variable in climate feedback analysis. J. Climate, 25, 2578-2582.

Held, I. M., and B. J. Soden, 2000: Water vapor feedback anad global warming. Annu. Rev. Energy Environ., 25, 441-475.
Held, I. M., and B. J. Soden, 2006: Robust Responses of the Hydrological Cycle to Global Warming. Journal of Climate, 19, 5686

Held, I. M., M. Ting, and H. Wang, 2002: Northern winter stationary waves: theory and modeling. J. Climate, 15, 2125-2144.

Heng, K., and P. Kopparla, 2012: On the Stability of Super-Earth Atmospheres. Astrophys. J., 754, 60.

Heng, K., and S. S. Vogt, 2010: Gliese 581g as a scaled-up version of Earth: atmospheric circulation simulations. ArXiv e-prints.

Heng, K., D. M. W. Frierson, and P. J. Phillipps, 2011a: Atmospheric circulation of tidally locked exoplanets: II. Dual-band radiative transfer and convective adjustment. Mon. Not. R. Astron. Soc., 418, 2669-2696.

Heng, K., K. Menou, and P. J. Phillipps, 2011b: Atmospheric circulation of tidally locked exoplanets: a suite of benchmark tests for dynamical solvers. Mon. Not. R. Astron. Soc., 413, 2380-2402.

Herrnstein, A., and T. E. Dowling, 2007: Effects of topography on the spin-up of a Venus atmospheric model. Journal of Geophysical Research (Planets), 112(E11).

Hide, R., 1969: Dynamics of the Atmospheres of the Major Planets with an Appendix on the Viscous Boundary Layer at the Rigid Bounding Surface of an Electrically-Conducting Rotating Fluid in the Presence of a Magnetic Field. J. Atmos. Sci., 26, 841-853.

Hoffman, P. F., and D. Schrag, 2002: Review article: The snowball Earth hypothesis: testing the limits of global change. Terra Nova, 14, 129-155.

Hollingsworth, J. L., R. E. Young, G. Schubert, C. Covey, and A. S. Grossman, 2007: A simple-physics global circulation model for Venus: Sensitivity assessments of atmospheric superrotation. Geophys. Res. Lett., 34, 5202.

Holton, J. R., 2004: An Introduction to Dynamic Meteorology, 4th Ed.. Academic Press, San Diego.

Hoskins, B., R. Neale, M. Rodwell, and G. Yang, 1999: Aspects of the large-scale tropical atmospheric circulation. Tellus Series $B$ Chemical and Physical Meteorology B, 51, 33-44.

Hoskins, B. J., and D. J. Karoly, 1981: The Steady Linear Response of a Spherical Atmosphere to Thermal and Orographic Forcing. Journal of Atmospheric Sciences, 38, 1179-1196.

Hu, Y., and F. Ding, 2011: Radiative constraints on the habitability of exoplanets Gliese 581c and Gliese 581d. Astron. Astrophys., 526, A135.

Huang, H.-P., and W. A. Robinson, 1998: Two-Dimensional Turbulence and Persistent Zonal Jets in a Global Barotropic Model. Journal of Atmospheric Sciences, 55, 611-632.

Hunt, B. G., 1979: The Influence of the Earth's Rotation Rate on the General Circulation of the Atmosphere. Journal of Atmospheric Sciences, 36, 1392-1408.

Ingersoll, A. P., 1969: The Runaway Greenhouse: A History of Water on Venus. Journal of Atmospheric Sciences, 26, 11911198.

Ingersoll, A. P., 1989: Io meteorology - How atmospheric pressure is controlled locally by volcanos and surface frosts. Icarus, $\mathbf{8 1}$, 298-313. 
Ingersoll, A. P., M. E. Summers, and S. G. Schlipf, 1985: Supersonic meteorology of Io - Sublimation-driven flow of SO2. Icarus, 64, 375-390.

Ingersoll, A. P., T. E. Dowling, P. J. Gierasch, G. S. Orton, P. L. Read, A. Sánchez-Lavega, A. P. Showman, A. A. SimonMiller, and A. R. Vasavada, 2004: Dynamics of Jupiter's atmosphere, pp. 105-128, Jupiter. The Planet, Satellites and Magnetosphere.

Ishioka, K., M. Yamada, Y.-Y. Hayashi, and S. Yoden, 1999: Pattern formation from two-dimensional decaying turbulence on a rotating sphere. Nagare Multimedia, The Japan Society of Fluid Mechanics, [available online at http://www.nagare.or.jp/mm/99/ishioka/].

Ishiwatari, M., S.-I. Takehiro, K. Nakajima, and Y.-Y. Hayashi, 2002: A Numerical Study on Appearance of the Runaway Greenhouse State of a Three-Dimensional Gray Atmosphere. Journal of Atmospheric Sciences, 59, 3223-3238.

Ishiwatari, M., K. Nakajima, S. Takehiro, and Y.-Y. Hayashi, 2007: Dependence of climate states of gray atmosphere on solar constant: From the runaway greenhouse to the snowball states. Journal of Geophysical Research (Atmospheres), 112(D11), 13,120.

Jansen, M., and R. Ferrari, 2012: Macroturbulent Equilibration in a Thermally Forced Primitive Equation System. Journal of Atmospheric Sciences, 69, 695-713.

Joshi, M., 2003: Climate Model Studies of Synchronously Rotating Planets. Astrobiology, 3, 415-427.

Joshi, M. M., and R. M. Haberle, 2012: Suppression of the Water Ice and Snow Albedo Feedback on Planets Orbiting Red Dwarf Stars and the Subsequent Widening of the Habitable Zone. Astrobiology, 12, 3-8.

Joshi, M. M., R. M. Haberle, and R. T. Reynolds, 1997: Simulations of the Atmospheres of Synchronously Rotating Terrestrial Planets Orbiting M Dwarfs: Conditions for Atmospheric Collapse and the Implications for Habitability. Icarus, 129, 450465.

Juckes, M. N., 2000: The static stability of the midlatitude troposphere: The relevance of moisture. J. Atmos. Sci., 57, 30503057.

Kaltenegger, L., A. Segura, and S. Mohanty, 2011: Model Spectra of the First Potentially Habitable Super-Earth - G1581d. Astrophys. J., 733, 35.

Karalidi, T., D. M. Stam, and J. W. Hovenier, 2012: Looking for the rainbow on exoplanets covered by liquid and icy water clouds. Astron. Astrophys., 548, A90.

Kaspi, Y., and T. Schneider, 2011: Downstream Self-Destruction of Storm Tracks. Journal of Atmospheric Sciences, 68, 24592464.

Kaspi, Y., and A. P. Showman, 2012: Three-dimensional Atmospheric Circulation and Climate of Terrestrial Exoplanets and Super Earths. AAS/Division for Planetary Sciences Meeting Abstracts, vol. 44 of AAS/Division for Planetary Sciences Meeting Abstracts, p. \#208.04.

Kasting, J. F., 1988: Runaway and moist greenhouse atmospheres and the evolution of earth and Venus. Icarus, 74, 472-494.

Kasting, J. F., D. P. Whitmire, and R. T. Reynolds, 1993: Habitable Zones around Main Sequence Stars. Icarus, 101, 108-128.
Kawahara, H., and Y. Fujii, 2011: Mapping Clouds and Terrain of Earth-like Planets from Photometric Variability: Demonstration with Planets in Face-on Orbits. Astrophys. J. Lett., 739, L62.

Kim, H.-K., and S. Lee, 2001: Hadley Cell Dynamics in a Primitive Equation Model. Part II: Nonaxisymmetric Flow. Journal of Atmospheric Sciences, 58, 2859-2871.

Kite, E. S., E. Gaidos, and M. Manga, 2011: Climate Instability on Tidally Locked Exoplanets. Astrophys. J., 743, 41.

Knutson, H. A., D. Charbonneau, L. E. Allen, J. J. Fortney, E. Agol, N. B. Cowan, A. P. Showman, C. S. Cooper, and S. T. Megeath, 2007: A map of the day-night contrast of the extrasolar planet HD 189733b. Nature, 447, 183-186.

Kombayashi, M., 1967: Discrete equilibrium temperatures of a hypothetical planet with the atmosphere and the hydrosphere of one component-two phase system under constant solar radiation. J. Meteor. Soc. Japan, 45, 137-138.

Kraucunas, I., and D. L. Hartmann, 2005: Equatorial Superrotation and the Factors Controlling the Zonal-Mean Zonal Winds in the Tropical Upper Troposphere. J. Atmos. Sci., 62, 371-389.

Kuo, A. C., and L. M. Polvani, 1997: Time-Dependent Fully Nonlinear Geostrophic Adjustment. Journal of Physical Oceanography, 27, 1614-1634.

Larichev, V. D., and I. M. Held, 1995: Eddy amplitudes and fluxes in a homogenous model of fully developed baroclinic instability. Journal of Physical Oceanography, 25, 2285-2297.

Le Hir, G., G. Ramstein, Y. Donnadieu, and Y. Goddéris, 2008: Scenario for the evolution of atmospheric pco2 during a snowball earth. Geology, 36(1), 47-50.

Le Hir, G., Y. Donnadieu, Y. Goddéris, R. T. Pierrehumbert, G. P. Halverson, M. Macouin, A. Nédélec, and G. Ramstein, 2009: The snowball earth aftermath: Exploring the limits of continental weathering processes. Earth and Planetary Science Letters, 277(3), 453-463.

Lebonnois, S., C. Covey, A. Grossman, H. Parish, G. Schubert, R. Walterscheid, P. Lauritzen, and C. Jablonowski, 2012: Angular momentum budget in General Circulation Models of superrotating atmospheres: A critical diagnostic. Journal of Geophysical Research (Planets), 117(E16), 12,004.

Leconte, J., F. Forget, B. Charnay, R. Wordsworth, F. Selsis, E. Millour, and A. Spiga, 2013: 3D climate modeling of closein land planets: Circulation patterns, climate moist bistability, and habitability. Astronomy and Astrophysics, in press.

Lee, C., S. R. Lewis, and P. L. Read, 2007: Superrotation in a Venus general circulation model. Journal of Geophysical Research (Planets), 112(E11).

Léger, A., et al., 2004: A new family of planets? "Ocean-Planets". Icarus, 169, 499-504.

Léger, A., et al., 2011: The extreme physical properties of the CoRoT-7b super-Earth. Icarus, 213, 1-11.

Leovy, C., 2001: Weather and climate on Mars. Nature, 412, 245-249.

Lindzen, R. S., and A. V. Hou, 1988: Hadley Circulations for Zonally Averaged Heating Centered off the Equator. Journal of Atmospheric Sciences, 45, 2416-2427. 
Lindzen, R. S., and S. Nigam, 1987: On the role of sea surface temperature gradients in forcing low-level winds and convergence in the Tropics. J. Atmos. Sci., 44, 2418-2436.

Lissauer, J. J., et al., 2011: A closely packed system of low-mass, low-density planets transiting Kepler-11. Nature, 470, 53-58.

Lopez, E. D., J. J. Fortney, and N. Miller, 2012: How Thermal Evolution and Mass-loss Sculpt Populations of Super-Earths and Sub-Neptunes: Application to the Kepler-11 System and Beyond. Astrophys. J., 761, 59.

Lorenz, E. N., 1955: Available potential energy and the maintenance of the general circulation. Tellus, 7, 157-167.

Lorenz, E. N., 1978: Available energy and the maintenance of a moist circulation. Tellus, 30, 15-31.

Lorenz, E. N., 1979: Numerical evaluation of moist available energy. Tellus, 31, 230-235.

Majeau, C., E. Agol, and N. B. Cowan, 2012: A Two-dimensional Infrared Map of the Extrasolar Planet HD 189733b. Astrophys. J. Lett., 747, L20.

Maltrud, M. E., and G. K. Vallis, 1993: Energy and enstrophy transfer in numerical simulations of two-dimensional turbulence. Physics of Fluids, 5, 1760-1775.

Marcus, P. S., and C. Lee, 1998: A model for eastward and westward jets in laboratory experiments and planetary atmospheres. Physics of Fluids, 10, 1474-1489.

Marshall, J., and R. A. Plumb, 2007: Atmosphere, Ocean, and Climate Dynamics. Academic Press.

Matsuno, T., 1966: Quasi-geostrophic motions in the equatorial area. J. Meteorol. Soc. Japan, 44, 25-43.

Mayor, M., et al., 2009: The HARPS search for southern extrasolar planets. XVIII. An Earth-mass planet in the GJ 581 planetary system. Astron. Astrophys., 507, 487-494.

McWilliams, J. C., 2006: Fundamentals of Geophysical Fluid Dynamics. Cambridge Univ. Press, Cambridge, UK.

Menou, K., and E. Rauscher, 2009: Atmospheric Circulation of Hot Jupiters: A Shallow Three-Dimensional Model. Astrophys. J., 700, 887-897.

Merlis, T. M., and T. Schneider, 2010: Atmospheric dynamics of Earth-like tidally locked aquaplanets. ArXiv e-prints.

Merlis, T. M., T. Schneider, S. Bordoni, and I. Eisenman, 2012: Hadley circulation response to orbital precession. Part I: Aquaplanets. J. Climate, p. in press.

Miller, S. L., 1953: A Production of Amino Acids under Possible Primitive Earth Conditions. Science, 117, 528-529.

Mitchell, J. L., and G. K. Vallis, 2010: The transition to superrotation in terrestrial atmospheres. Journal of Geophysical Research (Planets), 115(E14), 12,008.

Mitchell, J. L., R. T. Pierrehumbert, D. M. W. Frierson, and R. Caballero, 2006: The dynamics behind Titan's methane clouds. Proceedings of the National Academy of Science, 103, 18,42118,426 .

Mitchell, J. L., R. T. Pierrehumbert, D. M. W. Frierson, and R. Caballero, 2009: The impact of methane thermodynamics on seasonal convection and circulation in a model Titan atmosphere. Icarus, 203, 250-264.
Navarra, A., and G. Boccaletti, 2002: Numerical general circulation experiments of sensitivity to Earth rotation rate. Climate Dynamics, 19, 467-483.

Neelin, J. D., and I. M. Held, 1987: Modeling tropical convergence based on the moist static energy budget. Mon. Wea. Rev., 115, 3-12.

Nettelmann, N., J. J. Fortney, U. Kramm, and R. Redmer, 2011: Thermal Evolution and Structure Models of the Transiting Super-Earth GJ 1214b. Astrophys. J., 733, 2.

Niemann, H. B., et al., 2005: The abundances of constituents of Titan's atmosphere from the GCMS instrument on the Huygens probe. Nature, 438, 779-784.

Norton, W. A., 2006: Tropical Wave Driving of the Annual Cycle in Tropical Tropopause Temperatures. Part II: Model Results. J. Atmos. Sci., 63, 1420-1431.

Nozawa, T., and S. Yoden, 1997: Formation of zonal band structure in forced two-dimensional turbulence on a rotating sphere. Physics of Fluids, 9, 2081-2093.

O'Gorman, P. A., 2010: Understanding the varied response of the extratropical storm tracks to climate change. Proc. Nat. Aacad. Sci., 107, 19,176-19,180.

O'Gorman, P. A., 2011: The effective static stability experienced by eddies in a moist atmosphere. J. Atmos. Sci., 68, 75-90.

O'Gorman, P. A., and T. Schneider, 2008: The hydrological cycle over a wide range of climates simulated with an idealized GCM. J. Climate, 21, 3815-3832.

O’Gorman, P. A., R. P. Allan, M. P. Byrne, and M. Previdi, 2011: Energetic constraints on precipitation under climate change. Surv. Geophys., pp. 1-24.

Olbers, R. G., J. Willebrand, and C. Eden, 2011: Ocean Dynamics. Springer-Verlag, New York.

Pallé, E., E. B. Ford, S. Seager, P. Montañés-Rodríguez, and M. Vazquez, 2008: Identifying the Rotation Rate and the Presence of Dynamic Weather on Extrasolar Earth-like Planets from Photometric Observations. Astrophys. J., 676, 1319-1329.

Parish, H. F., G. Schubert, C. Covey, R. L. Walterscheid, A. Grossman, and S. Lebonnois, 2011: Decadal variations in a Venus general circulation model. Icarus, 212, 42-65.

Pavan, V., and I. M. Held, 1996: The diffusive approximation for eddy fluxes in baroclinically unstable flows. Journal of the Atmospheric Sciences, 53, 1262-1272.

Pedlosky, J., 1987: Geophysical Fluid Dynamics, 2nd Ed. Springer-Verlag, New York.

Pedlosky, J., 2003: Waves in the Ocean and Atmosphere. Springer-Verlag, New York.

Pedlosky, J., 2004: Ocean Circulation Theory. Springer-Verlag, New York.

Peixoto, J. P., and A. H. Oort, 1992: Physics of Climate. American Institute of Physics, New York.

Perna, R., K. Menou, and E. Rauscher, 2010: Magnetic Drag on Hot Jupiter Atmospheric Winds. Astrophys. J., 719, 14211426.

Perna, R., K. Heng, and F. Pont, 2012: The Effects of Irradiation on Hot Jovian Atmospheres: Heat Redistribution and Energy Dissipation. Astrophys. J., 751, 59. 
Pierrehumbert, R., and E. Gaidos, 2011: Hydrogen Greenhouse Planets Beyond the Habitable Zone. Astrophys. J. Lett., 734, L13.

Pierrehumbert, R. T., 1995: Thermostats, radiator fins, and the local runaway greenhouse. J. Atmos. Sci., 52, 1784-1806.

Pierrehumbert, R. T., 2002: The hydrologic cycle in deep-time climate problems. Nature, 419, 191-198.

Pierrehumbert, R. T., 2010: Principles of Planetary Climate. Cambridge University Press.

Pierrehumbert, R. T., 2011: A Palette of Climates for Gliese 581g. Astrophys. J. Lett., 726, L8.

Pierrehumbert, R. T., and K. L. Swanson, 1995: Baroclinic Instability. Annu. Rev. Fluid Mech., 27, 419-467.

Pierrehumbert, R. T., H. Brogniez, and R. Roca, 2007: On the relative humidity of the atmosphere. The Global Circulation of the Atmosphere, T. Schneider and A. H. Sobel, Eds., Princeton University Press, pp. 143-185.

Pierrehumbert, R. T., D. S. Abbot, A. Voigt, and D. Koll, 2011: Climate of the Neoproterozoic. Annual Review of Earth and Planetary Sciences, 39, 417-460.

Polvani, L. M., and A. H. Sobel, 2002: The Hadley Circulation and the Weak Temperature Gradient Approximation. Journal of Atmospheric Sciences, 59, 1744-1752.

Polvani, L. M., D. W. Waugh, and R. A. Plumb, 1995: On the Subtropical Edge of the Stratospheric Surf Zone. Journal of Atmospheric Sciences, 52, 1288-1309.

Pont, F., D. K. Sing, N. P. Gibson, S. Aigrain, G. Henry, and N. Husnoo, 2012: The prevalence of dust on the exoplanet HD 189733b from Hubble and Spitzer observations. ArXiv eprints.

Randel, W. J., and I. M. Held, 1991: Phase Speed Spectra of Transient Eddy Fluxes and Critical Layer Absorption. Journal of Atmospheric Sciences, 48, 688-697.

Rauscher, E., and K. Menou, 2010: Three-dimensional Modeling of Hot Jupiter Atmospheric Flows. Astrophys. J., 714, 13341342.

Rauscher, E., and K. Menou, 2012: The Role of Drag in the Energetics of Strongly Forced Exoplanet Atmospheres. Astrophys. $J ., 745,78$

Raymond, D. J., S. Sessions, A. H. Sobel, and Z. Fuchs, 2009: The mechanics of gross moist stability. J. Adv. Model. Earth Syst., 1, Art. \#9, 20 pp.

Read, P. L., 2011: Dynamics and circulation regimes of terrestrial planets. Planet. Space Sci., 59, 900-914.

Read, P. L., and S. R. Lewis, 2004: The Martian Climate Revisited. Springer/Praxis, New York.

Read, P. L., Y. H. Yamazaki, S. R. Lewis, P. D. Williams, R. Wordsworth, K. Miki-Yamazaki, J. Sommeria, and H. Didelle, 2007: Dynamics of Convectively Driven Banded Jets in the Laboratory. Journal of Atmospheric Sciences, 64, 4031.

Rennó, N. O., 1997: Multiple equilibria in radiative-convective atmospheres. Tellus Series A, 49, 423.

Rhines, P. B., 1975: Waves and turbulence on a beta-plane. Journal of Fluid Mechanics, 69, 417-443.
Richardson, L. J., D. Deming, K. Horning, S. Seager, and J. Harrington, 2007: A spectrum of an extrasolar planet. Nature, 445, 892-895.

Robinson, T. D., et al., 2011: Earth as an Extrasolar Planet: Earth Model Validation Using EPOXI Earth Observations. Astrobiology, 11, 393-408.

Rogers, L. A., and S. Seager, 2010: Three Possible Origins for the Gas Layer on GJ 1214b. Astrophys. J., 716, 1208-1216.

Rogers, L. A., P. Bodenheimer, J. J. Lissauer, and S. Seager, 2011: Formation and Structure of Low-density exo-Neptunes. Astrophys. J., 738, 59.

Romps, D. M., and Z. Kuang, 2010: Do undiluted convective plumes exist in the upper tropical troposphere? J. Atmos. Sci., 67, 468-484.

Salby, M. L., and R. R. Garcia, 1987: Transient Response to Localized Episodic Heating in the Tropics. Part I: Excitation and Short-Time Near-Field Behavior. Journal of Atmospheric Sciences, 44, 458-498.

Sanromá, E., and E. Pallé, 2012: Reconstructing the Photometric Light Curves of Earth as a Planet along Its History. Astrophys. J., 744, 188.

Saravanan, R., 1993: Equatorial Superrotation and Maintenance of the General Circulation in Two-Level Models. J. Atmos. Sci., 50, 1211-1227.

Sarmiento, J. L., and N. Gruber, 2006: Ocean biogeochemical dynamics. Princeton University Press.

Schneider, T., 2006: The General Circulation of the Atmosphere. Annual Review of Earth and Planetary Sciences, 34, 655-688.

Schneider, T., 2007: The thermal stratification of the extratropical troposphere. The Global Circulation of the Atmosphere, T. Schneider and A. H. Sobel, Eds., Princeton University Press, pp. 47-77.

Schneider, T., and S. Bordoni, 2008: Eddy-Mediated Regime Transitions in the Seasonal Cycle of a Hadley Circulation and Implications for Monsoon Dynamics. Journal of Atmospheric Sciences, 65, 915.

Schneider, T., and C. C. Walker, 2006: Self-Organization of Atmospheric Macroturbulence into Critical States of Weak Nonlinear Eddy Eddy Interactions. Journal of Atmospheric Sciences, 63, 1569-1586.

Schneider, T., and C. C. Walker, 2008: Scaling laws and regime transitions of macroturbulence in dry atmospheres. J. Atmos. Sci., 65, 2153-2173.

Schneider, T., P. A. O'Gorman, and X. J. Levine, 2010: Water vapor and the dynamics of climate changes. Reviews of Geophysics, 48, 3001.

Schumacher, C., R. A. Houze, Jr., and I. Kraucunas, 2004: The Tropical Dynamical Response to Latent Heating Estimates Derived from the TRMM Precipitation Radar. Journal of Atmospheric Sciences, 61, 1341-1358.

Scott, R. K., 2010: The structure of zonal jets in shallow water turbulence on the sphere. In IUTAM Symposium on Turbulence in the Atmosphere and Oceans (D.G. Dritschel, Ed.), pp. 243252 , Springer.

Scott, R. K., and D. G. Dritschel, 2012: The structure of zonal jets in geostrophic turbulence. J. Fluid Mech., 711, 576-598. 
Scott, R. K., and A.-S. Tissier, 2012: The generation of zonal jets by large-scale mixing. Physics of Fluids, 24(12), 126,601.

Seager, S., and D. Deming, 2010: Exoplanet Atmospheres. Annu. Rev. Astron. Astrophys., 48, 631-672.

Seager, S., D. Deming, and J. A. Valenti, 2008: Transiting Exoplanets with JWST. ArXiv e-prints.

Selsis, F., R. D. Wordsworth, and F. Forget, 2011: Thermal phase curves of nontransiting terrestrial exoplanets. I. Characterizing atmospheres. Astron. Astrophys., 532, A1.

Shell, K. M., and I. M. Held, 2004: Abrupt Transition to Strong Superrotation in an Axisymmetric Model of the Upper Troposphere. J. Atmos. Sci., 61, 2928-2935.

Sherwood, S. C., W. Ingram, Y. Tsushima, M. Satoh, M. Roberts, P. L. Vidale, and P. A. O'Gorman, 2010a: Relative humidity changes in a warmer climate. J. Geophys. Res., 115, 09,104.

Sherwood, S. C., R. Roca, T. M. Weckwerth, and N. G. Andronova, 2010b: Tropospheric water vapor, convection, and climate. Reviews of Geophysics, 48, RG2001.

Showman, A. P., and T. Guillot, 2002: Atmospheric circulation and tides of "51 Pegasus b-like" planets. Astron. Astrophys., 385, $166-180$.

Showman, A. P., and L. M. Polvani, 2010: The Matsuno-Gill model and equatorial superrotation. Geophys. Res. Lett., 37, 18,811 .

Showman, A. P., and L. M. Polvani, 2011: Equatorial Superrotation on Tidally Locked Exoplanets. Astrophys. J., 738, 71.

Showman, A. P., C. S. Cooper, J. J. Fortney, and M. S. Marley, 2008: Atmospheric Circulation of Hot Jupiters: Threedimensional Circulation Models of HD 209458b and HD 189733b with Simplified Forcing. Astrophys. J., 682, 559-576.

Showman, A. P., J. J. Fortney, Y. Lian, M. S. Marley, R. S. Freedman, H. A. Knutson, and D. Charbonneau, 2009: Atmospheric Circulation of Hot Jupiters: Coupled Radiative-Dynamical General Circulation Model Simulations of HD 189733b and HD 209458b. Astrophys. J., 699, 564-584.

Showman, A. P., J. Y.-K. Cho, and K. Menou, 2010: Atmospheric circulation of Exoplanets. In Exoplanets (S. Seager, Ed.), pp. 471-516, Univ. Arizona Press.

Showman, A. P., J. J. Fortney, N. K. Lewis, and M. Shabram, 2013: Doppler Signatures of the Atmospheric Circulation on Hot Jupiters. Astrophys. J., 762, 24.

Siedler, G., J. Church, and J. Gould, 2001: Ocean Circulation and Climate: Observing and Modeling the Global Ocean. Academic Press, International Geophysics Series, Vol. 77.

Sing, D. K., A. Vidal-Madjar, A. Lecavelier des Etangs, J. M. Desert, G. Ballester, and D. Ehrenreich, 2008: Determining atmospheric conditions at the terminator of the hot-Jupiter HD209458b. ArXiv e-prints, $\mathbf{8 0 3}$.

Sleep, N. H., and K. Zahnle, 2001: Carbon dioxide cycling and implications for climate on ancient earth. Journal of Geophysical Research: Planets (1991-2012), 106(E1), 1373-1399.

Smith, M. D., 2008: Spacecraft Observations of the Martian Atmosphere. Annual Review of Earth and Planetary Sciences, 36, 191-219.

Snellen, I. A. G., R. J. de Kok, E. J. W. de Mooij, and S. Albrecht, 2010: The orbital motion, absolute mass and high-altitude winds of exoplanet HD209458b. Nature, 465, 1049-1051.
Sobel, A. H., 2002: Water vapor as an active scalar in tropical atmospheric dynamics. Chaos, 12, 451-459.

Sobel, A. H., 2007: Simple models of ensemble-averaged tropical precipitation and surface wind, given the sea surface temperature. The Global Circulation of the Atmosphere, T. Schneider and A. H. Sobel, Eds., Princeton University Press, pp. 219-251.

Sobel, A. H., J. Nilsson, and L. M. Polvani, 2001: The Weak Temperature Gradient Approximation and Balanced Tropical Moisture Waves*. Journal of Atmospheric Sciences, 58, 36503665 .

Soden, B. J., and I. M. Held, 2006: An assessment of climate feedbacks in coupled ocean-atmosphere models. J. Climate, 19, 3354-3360.

Stevenson, D. J., 1999: Life-sustaining planets in interstellar space? Nature, 400, 32.

Stone, P. H., 1972: A simplified radiative-dynamical model for the static stability of rotating atmospheres. Journal of the Atmospheric Sciences, 29, 406-418.

Suarez, M. J., and D. G. Duffy, 1992: Terrestrial Superrotation: A Bifurcation of the General Circulation. J. Atmos. Sci., 49, 1541-1556.

Sukoriansky, S., N. Dikovskaya, and B. Galperin, 2007: On the "arrest" of inverse energy cascade and the Rhines scale. $J$. Atmos. Sci., 64, 3312-3327.

Thompson, A. F., and W. R. Young, 2006: Scaling Baroclinic Eddy Fluxes: Vortices and Energy Balance. Journal of Physical Oceanography, 36, 720.

Thompson, A. F., and W. R. Young, 2007: Two-layer baroclinic eddy heat fluxes: zonal flows and energy balance. J. Atmos. Sci., 64, 3214-3231.

Thompson, R. O. R. Y., 1971: Why There is an Intense Eastward Current in the North Atlantic but not in the South Atlantic. Journal of Physical Oceanography, 1, 235-238.

Thuburn, J., and G. C. Craig, 2000: Stratospheric influence on tropopause height: The radiative constraint. J. Atmos. Sci., 57, $17-28$.

Trenberth, K., and J. Caron, 2001: Estimates of meridional atmosphere and ocean heat transports. J. Climate, 14, 3433-3443.

Trenberth, K. E., J. T. Fasullo, and J. Kiehl, 2009: Earth's global energy budget. Bull. Amer. Meteor. Soc., 90, 311-323.

Tziperman, E., and B. Farrell, 2009: Pliocene equatorial temperature: Lessons from atmospheric superrotation. Paleoceanography, 24(26), A261,101+.

Udry, S., et al., 2007: The HARPS search for southern extra-solar planets. XI. Super-Earths (5 and $8 \mathrm{M}_{\oplus}$ ) in a 3-planet system. Astron. Astrophys., 469, L43-L47.

Vallis, G. K., 2006: Atmospheric and Oceanic Fluid Dynamics: Fundamentals and Large-Scale Circulation. Cambridge Univ. Press, Cambridge, UK.

Vallis, G. K., 2011: Climate and the Oceans. Princeton Primers in Climate series; Princeton Univ. Press.

Vallis, G. K., and R. Farneti, 2009: Meridional energy transport in the coupled atmosphere-ocean system: Scaling and numerical experiments. Quart. J. Roy. Meteor. Soc., 135, 1643-1660. 
Vallis, G. K., and M. E. Maltrud, 1993: Generation of mean flows and jets on a beta plane and over topography. J. Phys. Oceanography, 23, 1346-1362.

Vasavada, A. R., and A. P. Showman, 2005: Jovian atmospheric dynamics: an update after Galileo and Cassini. Reports of Progress in Physics, 68, 1935-1996.

von Paris, P., S. Gebauer, M. Godolt, J. L. Grenfell, P. Hedelt, D. Kitzmann, A. B. C. Patzer, H. Rauer, and B. Stracke, 2010: The extrasolar planet Gliese 581d: a potentially habitable planet? Astron. Astrophys., 522, A23.

Walker, C. C., and T. Schneider, 2005: Response of idealized Hadley circulations to seasonally varying heating. Geophys. Res. Lett., 32, L06,813.

Walker, C. C., and T. Schneider, 2006: Eddy Influences on Hadley Circulations: Simulations with an Idealized GCM. Journal of Atmospheric Sciences, 63, 3333-3350.

Walker, J. C. G., P. B. Hays, and J. F. Kasting, 1981: A negative feedback mechanism for the long-term stabilization of the earth's surface temperature. J. Geophys. Res., 86, 9776-9782.

Wiktorowicz, S. J., and A. P. Ingersoll, 2007: Liquid water oceans in ice giants. Icarus, 186, 436-447.

Williams, D. M., and D. Pollard, 2003: Extraordinary climates of Earth-like planets: three-dimensional climate simulations at extreme obliquity. International Journal of Astrobiology, 2, 119.

Williams, G. P., 1978: Planetary circulations. I - Barotropic representation of Jovian and terrestrial turbulence. Journal of Atmospheric Sciences, 35, 1399-1426.

Williams, G. P., 1988a: The dynamical range of global circulations - I. Climate Dynamics, 2, 205-260.

Williams, G. P., 1988b: The dynamical range of global circulations - II. Climate Dynamics, 3, 45-84.

Williams, G. P., and J. L. Holloway, 1982: The range and unity of planetary circulations. Nature, 297, 295-299.

Williams, R. G., and M. J. Follows, 2011: Ocean Dynamics and the Carbon Cycle. Cambridge Univ. Press, Cambridge, UK.

Wood, R. B., and M. E. McIntyre, 2010: A General Theorem on Angular-Momentum Changes due to Potential Vorticity Mixing and on Potential-Energy Changes due to Buoyancy Mixing. Journal of Atmospheric Sciences, 67, 1261-1274.

Wordsworth, R., 2012: Transient conditions for biogenesis on low-mass exoplanets with escaping hydrogen atmospheres. Icarus, 219, 267-273.

Wordsworth, R. D., P. L. Read, and Y. H. Yamazaki, 2008: Turbulence, waves, and jets in a differentially heated rotating annulus experiment. Physics of Fluids, 20(12), 126,602.

Wordsworth, R. D., F. Forget, F. Selsis, J.-B. Madeleine, E. Millour, and V. Eymet, 2010: Is Gliese 581d habitable? Some constraints from radiative-convective climate modeling. Astron. Astrophys., 522, A22.

Wordsworth, R. D., F. Forget, F. Selsis, E. Millour, B. Charnay, and J.-B. Madeleine, 2011: Gliese 581d is the First Discovered Terrestrial-mass Exoplanet in the Habitable Zone. Astrophys. J. Lett., 733, L48.

Xu, K.-M., and K. A. Emanuel, 1989: Is the tropical atmosphere conditionally unstable? Mon. Wea. Rev., 117, 1471-1479.
Yamamoto, M., and M. Takahashi, 2003: The Fully Developed Superrotation Simulated by a General Circulation Model of a Venus-like Atmosphere. Journal of Atmospheric Sciences, 60 , 561-574.

Yoden, S., K. Ishioka, Y.-Y. Hayashi, and M. Yamada, 1999: A further experiment on two-dimensional decaying turbulence on a rotating sphere. Nuovo Cimento C Geophysics Space Physics $C, 22,803-812$.

Zelinka, M. D., and D. L. Hartmann, 2010: Why is longwave cloud feedback positive? J. Geophys. Res., 115, D16,117.

Zurita-Gotor, P., and G. K. Vallis, 2009: Equilibration of baroclinic turbulence in primitive equations and quasigeostrophic models. J. Atmos. Sci., 66, 837-863. 